\title{
ZERO-TEMPERATURE LIMIT OF ONE-DIMENSIONAL GIBBS STATES VIA RENORMALIZATION: THE CASE OF LOCALLY CONSTANT POTENTIALS
}

\author{
J.-R. CHAZOTTES, J.-M. GAMBAUDO \& E. UGALDE
}

\begin{abstract}
Let $\mu_{\beta \phi}$ be the Gibbs state at "inverse temperature" $\beta$ of a locally constant potential $\phi: A^{\mathbb{Z}} \rightarrow \mathbb{R}$, where $A$ is a finite set). We prove that $\left(\mu_{\beta \phi}\right)_{\beta>0}$ converges when $\beta \rightarrow+\infty$, in weakstar topology, to a measure ('ground state') we characterize. We also estimate the 'speed' of this convergence. The limiting measure is concentrated on a certain subshift of finite type $\bar{X}(\phi)$ which in general is a finite union of transitive subshifts of finite type $\bar{X}_{J}(\phi)$.

The two main tools are approximation by periodic orbits and the Perron-Frobenius Theorem for matrices à la Birkhoff. The crucial idea we bring is a renormalization procedure which explains convergence and provides a recursive algorithm to compute the weights $\alpha_{J}$ of the ergodic decomposition of the limit. Each ergodic component is the unique equilibrium state of some potential we construct and it is supported on a $\bar{X}_{J}(\phi)$. This renormalization process generates a finite "tree" of SFT's, and an $\alpha_{J}$, which may be zero, is obtained as a product of weights along a branch of that tree.
\end{abstract}

\section{CONTENTS}

1. Introduction 2

2. Settings and Generalities 4

3. Preparatory results and reduction of the problem 6

4. Main Theorem 9

5. The Renormalization Lemma: Proof of Theorem 1

6. Examples 13

7. Proof of the Renormalization Lemma $\quad 17$

8. Auxiliary Lemmas 21

Appendix A. Proof of Proposition 1 (Periodic Approximation) 33

Appendix B. Auxiliary Inequalities of Lemma $1 \quad 36$

Appendix C. Projective Stability of the Eigensystems 46

Appendix D. Concentration of the Measure on the Heavy Components 47

$\begin{array}{ll}\text { References } & 49\end{array}$

Key words and phrases. Perron-Frobenius theorem, Birkhoff contraction coefficient, subshift of finite type, Markov chains.

This work is part of the project CrystalDyn funded by the Agence Nationale de la Recherche (ANR).. 


\section{INTRODUCTION}

We study a classical problem in equilibrium statistical mechanics, namely the description of the set of Gibbs states for a given potential as temperature changes and, more specifically, when it goes to zero. Already for classical lattice systems the zero-temperature limit is in general a very hard problem which is intimately related to ground states. Even knowing or assuming that the zero-temperature limit exists, the problem of determining which ground states are reached is very delicate. A systematic way of doing so, albeit for very special situations (roughly, when there are finitely many periodic ground states) is the so-called Pirogov-Sinai theory. We refer the reader to, e.g., $[7,14]$.

Somewhat surprisingly, there were only a few results until recently in the case of one-dimensional lattices, even for finite-range potentials. The problem we study can be formulated in the following way. We consider the space of two-sided sequences or configurations $A^{\mathbb{Z}}$, where $A$ is a finite set, and let $\phi: A^{\mathbb{Z}} \rightarrow \mathbb{R}$ be a locally constant potential. This means that there is an integer $r \geq 1$ such the values of $\phi$ coincide for all configurations with the same first $r+1$ symbols. Such a potential admits a unique Gibbs state which is none other than a $r$-step Markov measure with state space $A$. (When $r=1$, this is a usual Markov measure.) Ground states are probability measures achieving the maximum of the mapping $\nu \mapsto \int \phi d \nu$ where $\nu$ ranges over the set of shift-invariant probability measures on $A^{\mathbb{Z}}$. It is not difficult to prove that ground states of locally constant potentials are supported on (nonwandering) subshifts of finite type (hereafter SFT's). In particular, this implies that the set of ground states for a given locally constant potential is a finite-dimensional simplex. For, say, Hölder potentials, ground states can be for instance supported on a minimal uniquely ergodic subshift, see e.g. [6]. In general, one can have very complicated subshifts, e.g. minimal but with uncountably many invariant measures. Such SFT's have no reason to be transitive but are in general made of a finite number of transitive SFT's, each of them being possibly periodic. The question we are interested in is:

Question 1. For a locally constant potential $\phi$, does the limit (in weak-star topology) of $\left(\mu_{\beta \phi}\right)_{\beta>0}$, when $\beta \rightarrow+\infty$, exists ? Can it be precisely described? What can be expected is that the limit admits a certain barycentric decomposition among its ergodic (barycentric) ground states. But how to compute the corresponding weights?

Let us comment first on partial results or results in special cases. It can be proved [10] that, generically, the zero-temperature limit of Gibbs states of locally constant potentials is of the form $\left(\delta_{\mathbf{a}}+\cdots+\delta_{\sigma^{n-1} \mathbf{a}}\right) / n$ where $\sigma: A^{\mathbb{Z}} \rightarrow A^{\mathbb{Z}}$ is the shift and $\mathbf{a}$ is a periodic configuration with period $n\left(\sigma^{n} \mathbf{a}=\mathbf{a}\right)$. The zero-temperature limit of the one-dimensional Ising model is studied in detail in [7, Section 3.2]. In this model, $A=\{-,+\}$ and one can play with two parameters. Depending on their values, the zero-temperature limit can be, for instance, $\delta_{+}$, the Dirac measure on the 'all-+' configuration; or $\left(\delta_{-+}+\delta_{+-}\right) / 2$, where $\delta_{a b}$ stands for the Dirac measure on the periodic configuration $\cdots a b a b a b \cdots$; but it can also be the unique measure of maximal entropy of the SFT defined as the set of all configurations in $\{-,+\}^{\mathbb{Z}}$ with no two consecutive -'s. In all cases, one gets either the centroid of the ergodic ground states (when there are finitely many periodic ground configurations) or a measure of maximal entropy of a transitive SFT (when there are uncountably many ground configurations). In any case one gets a limiting measure equidistributing the mass among all the ground states. The simplest example where this is not true is given [8, Section 9], 
with an $A$ with three symbols. It is not incidental that three symbols (at least) are needed. Let us mention that in [11] ground states and their entropy are explicitly calculated for a very special class of one-dimensional lattice systems.

Regarding the above question, two previous works [3, 9] tackled it in its full generality. In [3], convergence is proved as a consequence of a general statement of Analytic Geometry (especially the theory of subanalytic maps). The limiting measure is not identified. In [9], a more explicit approach is used to prove convergence, and the limit is partially identified ${ }^{1}$. The weights of the barycentric decomposition of the limiting measure are not explicitly identified and the proof of convergence is somewhat indirect.

Let comment briefly on what makes the zero-temperature limit difficult to tackle. The point is that, in general, the support of the ground states of a given $\phi$ is not a transitive SFT: it is the finite disjoint union of irreducible SFT's $\bar{X}_{J}(\phi)$, each of them being possibly a (finite) union of topologically mixing SFT's cyclically permutated by the shift. Since the ground states of a given $\phi$ form a (finite-dimensional) simplex, there are finitely many extremal (ergodic) ground states. First, there is no obvious reason why convergence should take place. Second, the repartition of the mass among those SFT's is not at all evident.

In the present work, we offer an approach differing from $[3,9]$. It is based on two main tools and a new idea. These tools are approximation by periodic orbits and the contraction-mapping approach to the Perron-Frobenius theorem for matrices $[1,13]$. The new idea is a renormalization procedure which has to be iterated only a finite number of times (this is due to the fact that we consider locally constant potentials). Not only does this algorithm explain convergence but it also allows one to determine recursively the coefficients of the ergodic decomposition of the zero-temperature limit. Finally, we also obtain an exponentially decreasing bound in $\beta$ for the distance between $\mu_{\beta \phi}$ and its limit.

Colloquially, the renormalization works as follows. As mentioned above, any accumulation point $\nu$ of $\left(\mu_{\beta \phi}\right)_{\beta>0}$ is supported on a SFT when $\phi$ is locally constant. This SFT is in general not transitive but it is the (finite disjoint) union of transitive SFT's $\bar{X}_{J}(\phi)$. It is known that the support of $\nu$ contains an $\bar{X}_{K}(\phi)$ only if its toppological entropy is the maximal possible among all the $\bar{X}_{J}(\phi)$ 's. We shall call an 'heavy component' such a SFT and label them $1, \ldots, N_{\phi}$. In fact, to be an heavy component is a necessary but not sufficient condition. The renormalization consists in defining an SFT $\bar{X}^{\prime}(\phi)$ whose alphabet is obtained by labeling the heavy components by $\left\{1, \ldots, N_{\phi}\right\}$ and keeping only certain arrows between them. Correspondingly, one has to renormalize the potential $\phi$. Then we prove that the original problem is reduced to a new one where $\beta \phi$ is replaced by $\beta \phi^{\prime}+\psi^{\prime}$, where $\phi^{\prime}, \psi^{\prime}$ are locally constant potentials living on the SFT $\bar{X}^{\prime}(\phi)$. The additional potential $\psi^{\prime}$, independent of $\beta$, is a 'compensation factor'. This renormalization procedure is of course approximative and holds for $\beta$ large enough, but we are able to control the error term. This renormalization process generates a finite tree of SFT's, and a weight of the ergodic decomposition, which may be zero, is obtained as a product of weights along a branch of that tree.

We emphasize two crucial ingredients in the renormalization, namely the use of cohomology of potentials and the fact that we do not loose the Markovian character of the measure when we 'collapse' each heavy component.

\footnotetext{
${ }^{1}$ To be more precise, the author of [9] considers the case $\beta \phi+\psi$ with $\phi$ locally constant and $\psi$ Hölder continuous. But the presence of $\psi$ is not the issue.
} 
One may ask what happens beyond locally constant potentials. Even for a general Hölder continuous potential $\phi$, it is unknown at present if the $\operatorname{limit} \lim _{\beta \rightarrow+\infty} \mu_{\beta \phi}$ does exist or not. An indication of the complexity of this question is that the $\phi$-maximizing subshift can be very wild, for instance it can be a minimal non-uniquely ergodic subshift.

Let us make a few remarks on thermodynamic formalism and statistical mechanics. Above, we called 'ground states' the measures $\nu$ maximizing $\int \phi d \nu$. In statistical mechanics, ground states are rather those $\nu$ 's which minimize $\int \phi d \nu$. This is due to the fact that different sign conventions are used in the thermodynamic formalism [12]. If maximizing measures are connected with the limit $\beta \rightarrow+\infty$, minimizing measures are connected with the limit $\beta \rightarrow-\infty$. Physically, temperature (i.e. $\beta^{-1}$ ) is positive but mathematically it makes sense to consider it as a real number. Of course, it is enough to study the $\operatorname{limit}_{\beta \rightarrow+\infty} \mu_{\beta \phi}$ for any $\phi$. Another point is that a 'potential' in statistical mechanics is a family of shift-invariant functions $\Phi=\left(\Phi_{\Lambda}\right)$ indexed by finite subsets $\Lambda$ of $\mathbb{Z}$. What we call a potential here, that is a function $\phi: A^{\mathbb{Z}} \rightarrow \mathbb{R}$, is obtained from $\Phi$ by, e.g., $\phi=\sum_{\Lambda \ni 0} \Phi_{\Lambda} /|\Lambda|$. This makes sense if, for instance (see [12]), $\Phi$ is 'absolutely summable'. Let us notice that if $\phi$ is locally constant then $\Phi$ is of finite-range, and vice versa. The function $\phi$ has no name in statistical mechanics, although it naturally appears in the variational principle, and dynamicists are used to call it a 'potential'.

Scope of the article. In Section 2, we give some definitions and recall some general results about zero-temperature limits and ground states. In Section 3 we explain how Question 1 can be recast into a simpler one without loss of generality. In Section 4 we state the main result (Theorem 1 and the corresponding algorithm). We then prove the main result in Section 5 assuming the Renormalization Lemma whose proof is deferred to Section 7. The proof of this lemma relies on a certain number of technical Lemmas proved in Section 8. In turn, we postponed to Appendices $\mathrm{A}$ and $\mathrm{B}$ the proof of a number of statements used in the proof of the Lemmas. We have made a section with three examples (Section 6) to illustrate our result, in particular the renormalization procedure. Two of these examples seem to be new and we would not have found them without the renormalization idea. The first example was pointed out to us by an anonymous referee of a previous work in which we had attempted to tackle, without success, the zero-temperature limit problem. Indeed, this example was a counter-example to the result we had obtained and led us to guess the renormalization process.

\section{Settings and Generalities}

We consider a finite alphabet $A$ (with at least two elements) and let $A^{\mathbb{Z}}$ be the set of two-sided infinite sequences of symbols drawn from $A$. Elements of $A^{\mathbb{Z}}$ will be denoted by $\mathbf{a}, \mathbf{b}$, $\mathbf{c}$, etc. The shift map $\sigma: A^{\mathbb{Z}} \circlearrowleft$ is defined by $(\sigma \mathbf{a})_{i}=\mathbf{a}_{i}$ for all $i \in \mathbb{Z}$. We endow $A^{\mathbb{Z}}$ with the product topology which makes it a compact space. Given $\mathbf{b} \in A^{\mathbb{Z}}, p, q \in \mathbb{Z}, p \leq q$, we denote by $\left[\mathbf{b}_{q}^{p}\right.$ ] the cylinder set $\left\{\mathbf{a} \in A^{\mathbb{Z}}: \mathbf{a}_{p}=\mathbf{b}_{p}, \ldots, \mathbf{a}_{q}=\mathbf{b}_{q}\right\}$. 


\subsection{Equilibrium States and Pressure.}

We recall a few facts on equilibrium states. We refer the reader to, e.g., [2] and [12] for details.

Let $\phi: A^{\mathbb{Z}} \rightarrow \mathbb{R}$ be a continuous function. This means that $\operatorname{var}_{n} \phi \rightarrow 0$ as $n \rightarrow+\infty$, where $\operatorname{var}_{n} \phi:=\sup \left\{|\phi(\mathbf{a})-\phi(\mathbf{b})|: \mathbf{a}_{j}=\mathbf{b}_{j}, j=-n,-n+1, \ldots, 0, \ldots, n\right\}$ is the modulus of continuity of $\phi$. The equilibrium states of the 'potential' $\phi$ are those shift-invariant probability measures realizing the supremum of $h(\nu)+\int \phi d \nu$ among all shift-invariant probability measures $\nu$ (where $h(\nu)$ is the measure-theoretic entropy of $\nu$ ). The supremum equals $P(\phi)$, the topological pressure of $\phi$. With the weak-star topology, the set of shift-invariant probability measures is a Choquet simplex [12]. The set of equilibrium states of $\phi$ is a face of that simplex.

Let $Y \subset A^{\mathbb{Z}}$ be a subshift of $A^{\mathbb{Z}}$ (i.e., a closed shift-invariant subset of $A^{\mathbb{Z}}$ ) and $\psi: A^{\mathbb{Z}} \rightarrow \mathbb{R}$ be continuous. Then we can restrict $\psi$ to $Y$ and define its equilibrium states on $Y$. We denote the corresponding topological pressure by $P(\psi \mid Y)$, which we call the $\psi$-pressure on $Y$. When $\psi \equiv 0$, $P(\psi \mid Y)=h_{\mathrm{top}}(Y)$, the topological entropy of $Y$.

\subsection{Maximizing Measures and the Maximizing Subshift $\bar{X}$.}

For $\phi: A^{\mathbb{Z}} \rightarrow \mathbb{R}$ continuous, let

$$
\bar{\phi}:=\sup \left\{\int \phi d \nu: \nu \text { shift-invariant probability measure }\right\} .
$$

There always exists an invariant measure (by compactness) which realizes this supremum; we call it a maximizing measure for $\phi$. The shift-invariant, compact set containing the support of all $\phi$ maximizing measures is denoted by $\bar{X}=\bar{X}(\phi) \subset A^{\mathbb{Z}}$ and call it the $\phi$-maximizing subshift. The set of $\phi$-maximizing measures is a face of the Choquet simplex of shift-invariant probability measures, hence it is also a Choquet simplex. Its extreme points are the ergodic $\phi$-maximizing measures. It is easy to prove that

$$
\bar{\phi}=\sup _{p \in \mathbb{N}} \max _{\mathbf{a} \in \operatorname{Per}_{p}\left(A^{\mathbb{Z}}\right)} \frac{S_{p} \phi(\mathbf{a})}{p},
$$

where $S_{p} \phi(\mathbf{a}):=\sum_{i=0}^{p-1} \phi\left(\sigma^{i} \mathbf{a}\right)$ and where $\operatorname{Per}_{p}\left(A^{\mathbb{Z}}\right):=\left\{\mathbf{a} \in A^{\mathbb{Z}}: \sigma^{p} \mathbf{a}=\mathbf{a}\right\}(p \in \mathbb{N})$. Then

$$
\bar{X}=\bar{X}(\phi):=\operatorname{clos}\left(\bigcup_{p \in \mathbb{N}}\left\{\mathbf{a} \in \operatorname{Per}_{p}(X): S_{p} \phi(\mathbf{a})=p \bar{\phi}\right\}\right) \text {. }
$$

\subsection{Facts about the Zero-temperature Limit.}

Given a continuous $\phi$, we consider the one-parameter family of equilibrium states $\left\{\mu_{\beta \phi}: \beta>0\right\}$, where $\beta$ is interpreted in statistical physics as the inverse temperature. For each $\beta$, the potential $\beta \phi$ admits at least one equilibrium state.

We collect basic facts (that we will not need) relating zero-temperature limits of equilibrium states to maximizing measures in the following statement.

Facts. Let $\phi: A^{\mathbb{Z}} \rightarrow \mathbb{R}$ be a continuous potential. Then,

(1) $\left(\mu_{\beta \phi}\right)_{\beta>0}$ has a weak* accumulation point, as $\beta \rightarrow+\infty$. 
(2) Every accumulation point of $\left(\mu_{\beta \phi}\right)_{\beta>0}$ is a $\phi$-maximizing measure. Its support is contained in $\bar{X}=\bar{X}(\phi)$.

(3) Every accumulation point of $\left(\mu_{\beta \phi}\right)_{\beta>0}$ is of maximal entropy among $\phi$-maximizing measures.

Let us make a few comments on these facts. One can find the proofs of the above facts in [5]. Statement (1) results by compactness of the set of shift-invariant probability measures. Statement (2) is a straightfoward consequence of the variational principle. The last statement is also a consequence of the variational principle and of the convexity of $\beta \mapsto P(\beta \phi)$. In the formalism of statistical mechanics of lattice systems, these statements were known long before and can be found in [14, Appendix B.2]. The above proposition allows to conclude the existence of the zero-temperature limit only in special cases: when $\bar{X}$ supports a unique shift-invariant measure or when $\bar{X}$ has a unique measure of maximal entropy (e.g., when it is a transitive SFT).

A slight generalization of interest is to consider the case $\beta \phi+\psi$ where $\psi: A^{\mathbb{Z}} \rightarrow \mathbb{R}$ is another continuous potential. The above facts are valid with this new family of potentials if one replaces 'maximal entropy' by 'maximal $\psi$-pressure' in statement (3). Notice that $\bar{X}$ does not depend on $\psi$ but only on $\phi$.

\section{Preparatory Results and Reduction of the Problem}

\subsection{Locally Constant Potentials and Markov Measures.}

In this paper we restrict to locally constant potentials. We say that $\phi$ is locally constant if there exists a strictly positive integer $r$ such that

$$
\phi(\mathbf{a})=\phi(\mathbf{b}), \forall \mathbf{a}, \mathbf{b} \in A^{\mathbb{Z}} \text { such that } \mathbf{a} \in\left[\mathbf{b}_{0}^{r}\right] .
$$

We say that $\phi$ is a $(r+1)$-symbol potential. A $(r+1)$-symbol potential can be identified with a function from $A^{r+1}$ to $\mathbb{R}$ that we can still denote by $\phi$ by a slight abuse of notation. In that case, for each $\beta>0$, the potential $\beta \phi$ admits a unique equilibrium measure, which is also a ( $r$-step) Markov measure. (Notice that the case $r=0$ corresponds to the case of product measures for which the limit $\beta \rightarrow+\infty$ is trivial.)

Without loss of generality, we can reduce our problem to the case of (1-step) Markov measures, i.e., to the case of 2-symbol potentials. Let us make this precise.

\subsection{Recodification for Locally Constant Potentials, Maximizing SFT and Heavy Com- ponents.}

Let $\Phi, \Psi: A^{r+1} \rightarrow \mathbb{R}$ be $(r+1)$-symbol potentials and let $\mathcal{A}$ be the alphabet of words of length $r+1$ in the alphabet $A$. Then $A^{\mathbb{Z}}$ can be recoded as a topological Markov chain (hereafter TMC) $X \subset \mathcal{A}^{\mathbb{Z}}$ (that is, a SFT which can defined by words of length two).

We identify $X$ with the set of all bi-infinite paths on a directed graph $\mathcal{G}_{X}:=(\mathcal{A}, \mathcal{E})$, with vertex set $\mathcal{A}$ and arrow set $\mathcal{E}:=\left\{\left(a, a^{\prime}\right) \in \mathcal{A} \times \mathcal{A}:\left[a a^{\prime}\right] \cap X \neq \emptyset\right\}$. In this representation, the potentials $\Phi, \Psi$ become 'arrow functions' (2-symbol potentials) $\phi, \psi: \mathcal{E} \rightarrow \mathbb{R}$.

An elementary circuit is a cyclic path in $\mathcal{G}_{X}$ with no repeated vertices. Any circuit $C$ in $\mathcal{G}_{X}$ can be represented as a sum of elementary circuits $C=C_{1}+C_{2}+\cdots+C_{n}$, where all the arrows in $C$ 
appear in one and only one of the elementary circuits $C_{i}, 1 \leq i \leq n$, whence $|C|=\sum_{i=1}^{n}\left|C_{i}\right|$. In general, this representation is not unique.

To each periodic orbit $\mathbf{a} \in \operatorname{Per}_{p}(X)$ it corresponds a cyclic path $C(\mathbf{a}):=\left(\mathbf{a}_{0}, \mathbf{a}_{1}, \ldots, \mathbf{a}_{p-1}\right)$ in $\mathcal{G}_{X}$. This path can be represented as the sum of elementary circuits $C(\mathbf{a})=C_{1}+\cdots+C_{n(\mathbf{a})}$. Using this representation we can write $S_{p} \phi(\mathbf{a}) \equiv \phi(C(\mathbf{a})):=\sum_{i=1}^{n(\mathbf{a})} \phi\left(C_{i}\right)$, where $\phi(C):=\sum_{i=0}^{|C|-1} \phi\left(b_{i}, b_{i+1}\right)$ for each circuit $C=\left(b_{0}, \ldots, b_{|C|-1}\right)$ in $\mathcal{G}_{X}$. Since $\sum_{i=1}^{n}\left|C_{i}\right|=|C(\mathbf{a})|=p$, it follows that

$$
\begin{aligned}
\bar{\phi} & =\sup _{p \in \mathbb{N}} \max _{\mathbf{a} \in \operatorname{Per}_{p}(X)} \frac{S_{p} \phi(\mathbf{a})}{p}=\sup _{p \in \mathbb{N}} \max _{\mathbf{a} \in \operatorname{Per} p(X)} \frac{\phi(C(\mathbf{a})}{|C(\mathbf{a})|}=\sup _{p \in \mathbb{N}} \max _{\mathbf{a} \in \operatorname{Per}_{p}(A)} \sum_{i=1}^{n} \frac{\left|C_{i}\right|}{|C(\mathbf{a})|} \frac{\phi\left(C_{i}\right)}{\left|C_{i}\right|} \\
& =\max \left\{\frac{\phi(C)}{|C|}: C \text { is an elementary circuit in } \mathcal{G}_{X}\right\} .
\end{aligned}
$$

Notice that the set of elementary circuits in $\mathcal{G}_{X}$ has cardinality bounded by $(\# \mathcal{A})$ !.

The maximizing subshift $\bar{X}$ is such that $S_{p} \phi(\mathbf{a})=p \bar{\phi}$ for all $p \in \mathbb{N}$ and for all $\mathbf{a} \in \operatorname{Per}_{p}(\bar{X})$, i.e, $\bar{X}$ is the smallest subshift containing all the periodic points corresponding to maximizing circuits in $\mathcal{G}_{X}$. Let $\overline{\mathcal{C}}:=\left\{C\right.$ circuit in $\left.\mathcal{G}_{X}: \phi(C) /|C|=\bar{\phi}\right\}$, be the set of all maximizing circuits in $\mathcal{G}_{X}$, and let $\overline{\mathcal{C}}_{0} \subset \overline{\mathcal{C}}$ denote the set of all elementary maximizing circuits. Notice that any maximizing circuit in $\mathcal{G}_{X}$ is necessarily the sum of circuits in $\overline{\mathcal{C}}_{0}$. On the other hand, if all the arrows in a circuit $C=\left(b_{0}, \ldots, b_{|C|-1}\right)$ appear in a maximizing circuit, then necessarily $\phi(C) /|C|=\bar{\phi}$. Taking this into account it can be readily deduced that

$$
\bar{X}=\bar{X}(\phi)=\left\{\mathbf{a} \in X: \forall i \in \mathbb{Z}, \exists C_{i} \in \overline{\mathcal{C}}_{0} \text { such that }\left(\mathbf{a}_{i}, \mathbf{a}_{i+1}\right) \text { occurs in } C_{i}\right\} .
$$

It is clearly a (non-wandering) subshift of finite type defined by a subgraph $\mathcal{G}_{\bar{X}}:=(\overline{\mathcal{A}}, \overline{\mathcal{E}}) \prec$ $\mathcal{G}_{X}$, where $\overline{\mathcal{E}}$ is the arrow set spanning the maximizing circuits in $\mathcal{G}_{X}$ and where $\overline{\mathcal{A}}$ is the set of corresponding vertices.

The maximizing SFT $\bar{X}$ is a disjoint union of transitive subshifts and we set $\bar{X}=\bigcup_{J=0}^{N} \bar{X}_{J}$. To each transitive component $\bar{X}_{J}$ it corresponds a transitive subgraph $\mathcal{G}_{J}:=\left(\overline{\mathcal{A}}_{J}, \overline{\mathcal{E}}_{J}\right) \prec \mathcal{G}_{X}$. Let us order these transitive components so that $P\left(\psi \mid \bar{X}_{J}\right)=P(\psi \mid \bar{X})$ for each $1 \leq J \leq N_{\phi}$ and $P\left(\psi \mid \bar{X}_{J}\right)<P(\psi \mid \bar{X})$ for each $N_{\phi}<J \leq N$. (Recall that $P(\psi \mid \bar{X})=\max \left\{P\left(\psi \mid \bar{X}_{J}\right): 1 \leq J \leq N\right\}$.)

Definition 1 (Heavy components). We will refer to the SFT's $\bar{X}_{J}$ in the subcollection $\left\{\bar{X}_{J} \subset \bar{X}\right.$ : $\left.1 \leq J \leq N_{\phi}\right\}$ as the heavy components of $\bar{X}$. We denote by

$$
\overline{\mathcal{E}}_{\phi}:=\bigsqcup_{J=1}^{N_{\phi}} \overline{\mathcal{E}}_{J} \subset \overline{\mathcal{E}}
$$

the collection of all arrows in digraphs associated to heavy components.

As we shall see, the zero-temperature limit is concentated only on some of the heavy components.

From now on, and without loss of generality, we assume that we are given a directed graph $\mathcal{G}_{X}:=$ $(\mathcal{A}, \mathcal{E})$ and $\phi, \psi: \mathcal{E} \rightarrow \mathbb{R}$. 


\subsection{Transition Matrices and Equilibrium States.}

Let $\phi, \psi: \mathcal{E} \rightarrow \mathbb{R}$. To each $\beta \in \mathbb{R}$ it corresponds a unique equilibrium state $\mu_{\beta \phi+\psi}$, which is a (1step) Markov measure completely determined by the irreducible matrix $\mathcal{M}_{\beta \phi+\psi}: \mathcal{A} \times \mathcal{A} \rightarrow[0, \infty)$ defined by

$$
\mathcal{M}_{\beta \phi+\psi}\left(a, a^{\prime}\right)= \begin{cases}e^{\beta \phi\left(a, a^{\prime}\right)+\psi\left(a, a^{\prime}\right)} & \text { if }\left(a, a^{\prime}\right) \in \mathcal{E} \\ 0 & \text { otherwise }\end{cases}
$$

According to Perron-Frobenius Theorem (Theorem 2 in Appendix A) there are unique left and right maximal eigenvectors $\mathbf{v}_{\beta \phi+\psi}$ and $\mathbf{w}_{\beta \phi+\psi}$ associated to the maximal eigenvalue $\rho_{\beta \phi+\psi}:=$ $\max \left|\operatorname{spec}\left(\mathcal{M}_{\beta \phi+\psi}\right)\right|$, normalized such that $\mathbf{w}_{\beta \phi+\psi}^{\dagger} \mathbf{v}_{\beta \phi+\psi}=1$. We have the formula

$$
\mu_{\beta \phi+\psi}\left[\mathbf{b}_{0}^{n}\right]=\frac{1}{\rho_{\beta \phi+\psi}^{n+1}} \mathbf{w}_{\beta \phi+\psi}\left(\mathbf{b}_{0}\right)\left(\prod_{i=0}^{n-1} \mathcal{M}_{\beta \phi+\psi}\left(\mathbf{b}_{i}, \mathbf{b}_{i+1}\right)\right) \mathbf{v}_{\beta \phi+\psi}\left(\mathbf{b}_{n}\right),
$$

for every $\mathbf{b}_{0}^{n} \in X\left(n \in \mathbb{N}_{0}\right)$.

For each $1 \leq J \leq N, \overline{\mathcal{A}}_{J} \subset \mathcal{A}$ denote the vertex set of the digraph $\mathcal{G}_{J}:=\left(\overline{\mathcal{A}}_{J}, \overline{\mathcal{E}}_{J}\right)$ associated to the transitive component $\bar{X}_{J} \subset \bar{X}$. The transition matrices $\mathcal{M}_{\psi, J}: \overline{\mathcal{A}}_{J} \times \overline{\mathcal{A}}_{J} \rightarrow \mathbb{R}^{+}$defined by

$$
\mathcal{M}_{\psi, J}\left(a, a^{\prime}\right)= \begin{cases}e^{\psi\left(a, a^{\prime}\right)} & \text { if }\left(a, a^{\prime}\right) \in \overline{\mathcal{E}}_{J} \\ 0 & \text { otherwise }\end{cases}
$$

are irreducible, therefore they have associated to their maximal eigenvalue $\rho_{\psi, J}:=\max \left|\operatorname{spec}\left(\mathcal{M}_{\psi, J}\right)\right|$ unique left and right eigenvectors $\mathbf{v}_{\psi, J}, \mathbf{w}_{\psi, J}: \overline{\mathcal{A}}_{J} \rightarrow \mathbb{R}^{+}$such that $\mathbf{w}_{\psi, J}^{\dagger} \mathbf{v}_{\psi, J}=1$. We can associate to each transitive component $\bar{X}_{J} \subset \bar{X}$ the Markov measure $\nu_{\psi, J}$ defined by

$$
\nu_{\psi, J}\left[\mathbf{b}_{0}^{n}\right]:=\frac{1}{\rho_{\psi, J}^{n+1}} \mathbf{w}_{\psi, J}\left(\mathbf{b}_{0}\right)\left(\prod_{i=0}^{n-1} \mathcal{M}_{\psi, J}\left(\mathbf{b}_{i}, \mathbf{b}_{i+1}\right)\right) \mathbf{v}_{\psi, J}\left(\mathbf{b}_{n}\right),
$$

for each $\mathbf{b} \in X$ such that $\left[\mathbf{b}_{0}^{n}\right] \cap \bar{X}_{J} \neq \emptyset$. This is precisely the equilibrium state on $\bar{X}_{J}$ associated to the potential $\psi \mid \bar{X}_{J}$. We recall that $P\left(\psi \mid \bar{X}_{J}\right)=\log \rho_{\psi, J}$.

Our original question can be recast in the following way. We slightly generalize it and consider potentials of the form $\beta \phi+\psi$ which will appear naturally when we make the renormalization described in the next section.

Question 2 (Recasting Question 1). Let $X \subset \mathcal{A}^{\mathbb{Z}}$ be a topological Markov chain defined by the finite alphabet $\mathcal{A}$ and the arrow set $\mathcal{E}$ and let $\phi, \psi: \mathcal{E} \rightarrow \mathbb{R}$ be (2-symbol) potentials. Denote by $\mu_{\beta \phi+\psi}$ the unique equilibrium state of $\beta \phi+\psi$ for each $\beta>0$, which is a Markov measure.

Does the limit of $\left(\mu_{\beta \phi+\psi}\right)_{\beta>0}$ exists in weak-star topology? Can we compute its barycentric decomposition on the finite-dimensional simplex generated by the ergodic $\phi$-maximizing measures?

\subsection{Normalization of $\phi$ and $\psi$.}

It will be convenient to assume that $\bar{\phi}=P(\psi \mid \bar{X})=0$. If it is not the case, then one can redefine $\phi$ as $\phi-\bar{\phi}$. These potentials are cohomologous and yield the same Gibbs state $\mu_{\beta \phi}$. Moreover, this does not change the $\phi$-maximizing SFT $\bar{X}$. One can also redefine $\psi$ as $\psi-P(\psi \mid \bar{X})$ without changing the equilibrium states $\nu_{\psi, J}$. Finally, for any $\beta$, the normalized potentials do not change the equilibrium state $\mu_{\beta \phi+\psi}$. Since heavy components (Definition 1) maximize $P\left(\psi \mid \bar{X}_{J}\right), J=1 \ldots N$, 
and since $P(\psi \mid \bar{X})=\max \left\{P\left(\psi \mid \bar{X}_{J}\right): 1 \leq J \leq N\right\}$, then, if $P(\psi \mid \bar{X})=0$, we have $P\left(\psi \mid \bar{X}_{J}\right)<0$, $N_{\phi}<J \leq N$. Since $P\left(\psi \mid \bar{X}_{J}\right)=\log \rho_{\psi, J}$, this means that $\rho_{\psi, J} \leq 1$ for each $1 \leq J \leq N$, and $\rho_{\psi, J}=1$ for the heavy components $1 \leq J \leq N_{\phi}$.

\subsection{Notations.}

Henceforth, we will use the following convenient short-hand notations:

$$
\begin{gathered}
a= \pm b \quad \text { for } \quad-b \leq a \leq b \\
a=e^{ \pm b} \quad \text { for } \quad e^{-b} \leq a \leq e^{b}
\end{gathered}
$$

where $a, b$ are positive real numbers.

\section{Main Theorem}

We formulate our main result (Theorem 1) which answers Question 2 (hence Question 1). For convenience, the algorithm describing how to compute the zero-temperature limit is in Subsection 4.2 .

\subsection{Convergence when temperature goes to zero.}

Theorem 1. Let $X \subset \mathcal{A}^{\mathbb{Z}}$ be a topological Markov chain defined by the finite alphabet $\mathcal{A}$ and the arrow set $\mathcal{E}$ and let $\phi, \psi: \mathcal{E} \rightarrow \mathbb{R}$ be potentials. Denote by $\mu_{\beta \phi+\psi}$ the unique equilibrium state of $\beta \phi+\psi$ for each $\beta>0$. Let $\bar{X}$ be the $\phi$-maximizing SFT and $\bar{X}_{1}, \ldots, \bar{X}_{N_{\phi}} \subset \bar{X}$ its heavy components. For each heavy component $\bar{X}_{J}, 1 \leq J \leq N_{\phi}$, let $\nu_{\psi, J}$ be the (unique) equilibrium state on $\bar{X}_{J}$ associated to the potential $\psi$ (restricted to $\bar{X}_{J}$ ) and such that $\nu_{\psi, J}\left(\bar{X}_{J}\right)=1$.

Then the sequence $\left(\mu_{\beta \phi+\psi}\right)_{\beta>0}$ converges in the weak-star topology and

$$
\lim _{\beta \rightarrow+\infty} \mu_{\beta \phi+\psi}=\sum_{J=1}^{N_{\phi}} \alpha_{J} \nu_{\psi, J}:=\nu
$$

where $0 \leq \alpha_{J} \leq 1$ and $\sum_{J=1}^{N_{\phi}} \alpha_{J}=1$.

The $\alpha_{J}$ 's can be computed by means of a recursive algorithm, detailed below, which converges after a finite number of steps. Notice that some of the $\alpha_{J}$ 's may be zero (i.e., some heavy components can die out).

Furthermore, there exist $\beta_{0}=\beta_{0}(\mathcal{A}, \phi, \psi)$ and $C=C(\mathcal{A}, \phi)>0$ (independent of $\psi$ ) such that for all $\beta \geq \beta_{0}$

$$
\mu_{\beta \phi+\psi}\left[\mathbf{b}_{0}^{n}\right]=\alpha_{J} \nu_{\psi, J}\left[\mathbf{b}_{0}^{n}\right] \pm e^{-C \beta},
$$

whenever $\left[\mathbf{b}_{0}^{n}\right] \cap \bar{X}_{J} \neq \emptyset$ for some $1 \leq J \leq N_{\phi}$. 
Remark 1. The weak-star topology on the space of shift-invariant probability measures can be metrized by the distance

$$
\operatorname{dist}^{*}(\lambda, \eta)=\sum_{n=1}^{+\infty} \frac{1}{2^{n}} \sum_{b_{0}^{n-1} \in \mathcal{A}^{n}}\left|\lambda\left[b_{0}^{n-1}\right]-\eta\left[b_{0}^{n-1}\right]\right| .
$$

Hence (6) means that $\operatorname{dist}^{*}\left(\mu_{\beta \phi+\psi}, \nu\right) \leq e^{-C \beta}$ for $\beta$ large enough.

\subsection{The Algorithm to compute the zero-tempeature limit.}

To describe the algorithm for computing the coefficients $\alpha_{J}$, we need more notations and definitions. We assume that $\bar{\phi}=P(\psi \mid \bar{X})=0$ (see Subsection 3.4).

Definition 2 (Renormalized SFT). To each heavy component $\bar{X}_{J}, 1 \leq J \leq N_{\phi}$, it corresponds a sub-alphabet $\overline{\mathcal{A}}_{J} \subset \mathcal{A}$ such that $\bar{X}_{J} \subset \overline{\mathcal{A}}_{J}^{\mathbb{Z}}$. We define the renormalized alphabet $\mathcal{A}^{\prime}:=\left\{1,2, \ldots, N_{\phi}\right\}$, and the arrow set $\mathcal{E}^{\prime} \subset \mathcal{A}^{\prime} \times \mathcal{A}^{\prime}$ defined as follows: $(J, K) \in \mathcal{E}^{\prime}$ if and only if there exists $\mathbf{a} \in X$ and an $n \in \mathbb{N}$ such that $\mathbf{a}_{0} \in \overline{\mathcal{A}}_{J}, \mathbf{a}_{n} \in \overline{\mathcal{A}}_{K}$ and $\bigcup_{L=1}^{N_{\phi}}\left(\left\{\mathbf{a}_{m}: 1<m<n\right\} \cap \overline{\mathcal{A}}_{L}\right)=\emptyset$. The renormalized SFT is defined by

$$
X^{\prime}:=\left\{\mathbf{a} \in \mathcal{A}^{\prime \mathbb{Z}}:\left(\mathbf{a}_{n}, \mathbf{a}_{n+1}\right) \in \mathcal{E}^{\prime}, \forall n \in \mathbb{Z}\right\}
$$

Let $\mathcal{M}_{\psi}: \mathcal{A} \times \mathcal{A} \rightarrow \mathbb{R}^{+}$be defined in the same way as $\mathcal{M}_{\beta \phi+\psi}$, with $\beta \phi+\psi$ instead of $\psi$, and let $\overline{\mathcal{M}}_{\psi}$ be the restriction of $\mathcal{M}_{\psi}$ to all the transitive components of $\bar{X}$, either heavy or not. It is easy to verify that $\sum_{k=0}^{\infty} \overline{\mathcal{M}}_{\psi}^{k}(b, b)<\infty$, for each $b \in \mathcal{A} \backslash \bigsqcup_{J=1}^{N_{\phi}} \overline{\mathcal{A}}_{J}$.

We need some notations for paths in digraphs.

Notations (Paths in digraphs). Given two vertices $a, c$ in a digraph $\mathcal{G}$, we denote by $a \rightsquigarrow c$ any finite path starting from $a$ and ending at $c$. When the path is elementary (i.e., it does not contain any circuit) we write $a \rightarrow c$. The symbols ' $\rightsquigarrow$ ' and ' $\rightarrow$ ' will be naturally used as variables in path-depending functions. We will also use the notation $b \circlearrowleft$ to denote a finite circuit based on $b$.

Given $a, c \in \mathcal{A}$, we denote by $\operatorname{Path}[a, c]$ the collection of all elementary paths in $\mathcal{G}_{X}$ (the digraph defining $X)$ going from $a$ to $c$. To a given path of the form $a \rightarrow c=\left(a, b_{1}, \ldots, b_{m}, c\right) \in \operatorname{Path}[a, c]$, with $m \in \mathbb{N}$ and $b_{i} \in \mathcal{A} \backslash \bigsqcup_{J=1}^{N_{\phi}} \overline{\mathcal{A}}_{J}(1 \leq i \leq m)$ we associate the following transition pressure

$$
P_{\psi}(a \rightarrow c):=\log \left(\sum_{i=1}^{m} \sum_{k=0}^{\infty} \overline{\mathcal{M}}_{\psi}^{k}\left(b_{i}, b_{i}\right)\right)<+\infty .
$$

Let us now fix, for each heavy component $1 \leq J \leq N_{\phi}$, a central vertex $c_{J} \in \overline{\mathcal{A}}_{J}$. Since $\phi(C)=0$ for each circuit $C$ in $\mathcal{G}_{J}$ (the digraph defining $\left.\bar{X}_{J}\right)$, it follows that $\phi\left(c_{J} \rightsquigarrow a\right)$ has the same value for all paths $c_{J} \rightsquigarrow a$ in $\mathcal{G}_{J}$ connecting $c_{J}$ to $a$. Therefore we can define, for each $a \in \overline{\mathcal{A}}_{J}$, the central term

$$
\overleftarrow{\phi}(a):=\phi\left(c_{J} \rightsquigarrow a\right) \text { with } c_{J} \rightsquigarrow a \text { a path in } \mathcal{G}_{J} \text { from } c_{J} \text { to } a
$$


For $a \in \overline{\mathcal{A}}_{J}$ and $c \in \overline{\mathcal{A}}_{K}$, let $\widetilde{\operatorname{Path}}[a, c]$ be the set of all elementary paths in $\mathcal{G}_{X}$, starting at $a$ and ending at $c$, with no arrows in $\overline{\mathcal{E}}_{\phi}$ other than $a$ and $c$. We define the transition term

$$
\vec{\phi}(a, c):=\max \{\phi(a \rightarrow c): a \rightarrow c \in \widetilde{\operatorname{Path}}[a, c]\},
$$

where $\phi(a \rightarrow c):=\phi\left(a^{\prime}, b_{1}\right)+\sum_{i=1}^{m-1} \phi\left(b_{i}, b_{i+1}\right)+\phi\left(b_{m}, c\right)$ for $a \rightarrow c=\left(a, b_{1}, \ldots, b_{m}, c\right)$.

Definition 3 (Renormalized potentials). With the notations just introduced, we define the renormalized potentials $\phi^{\prime}, \psi^{\prime}: \mathcal{E}^{\prime} \rightarrow \mathbb{R}$ by

$$
\begin{aligned}
\phi^{\prime}(J, K) & :=\max _{a \in \overline{\mathcal{A}}_{J}, c \in \overline{\mathcal{A}}_{K}}\{\overleftarrow{\phi}(a)+\vec{\phi}(a, c)-\overleftarrow{\phi}(c)\} \\
\psi^{\prime}(J, K) & :=\log \left(\sum_{a, c \in \overline{\mathcal{A}}_{J, K}} \mathbf{v}_{\psi, J}(a) \mathbf{w}_{\psi, K}(c) \sum_{a \rightarrow c \in \overline{\operatorname{Path}}[a, c]} \exp \left(\psi(a \rightarrow c)+P_{\psi}(a \rightarrow c)\right)\right)
\end{aligned}
$$

where $\overline{\mathcal{A}}_{J, K} \subset \overline{\mathcal{A}}_{J} \times \overline{\mathcal{A}}_{K}$ denotes the set where $\tilde{\phi}(a, c):=\overleftarrow{\phi}(a)+\vec{\phi}(a, c)-\overleftarrow{\phi}(c)$ is maximal, $\overline{\mathrm{Path}}[a, c]$ the set of all simple paths from a to $c$ maximizing $\phi$, and $P_{\psi}(a \rightarrow c)$ is defined in (7).

Algorithm for the $\alpha_{J}$ 's (Continuation of Theorem 1).

(1) Let $X^{\prime} \subset \mathcal{A}^{\prime} \mathbb{Z}$ be the renormalized subshift, and $\phi^{\prime}, \psi^{\prime}: \mathcal{E}^{\prime} \rightarrow \mathbb{R}$ the renormalized two-symbol potentials defined above.

(2) Compute $\bar{X}^{\prime} \subset X^{\prime}$, the $\phi^{\prime}-$ maximizing SFT, and normalize $\phi^{\prime}$, i.e., replace $\phi^{\prime}$ by $\phi^{\prime}-\bar{\phi}^{\prime}$.

(3) Normalize $\psi^{\prime}$, i.e., replace $\psi^{\prime}$ by $\psi^{\prime}-P\left(\psi^{\prime} \mid \bar{X}^{\prime}\right)$, and identify the heavy components $\bar{X}_{1}^{\prime}, \ldots, \bar{X}_{N_{\phi^{\prime}}}^{\prime} \subset$ $\bar{X}^{\prime}$.

(4) Compute, for each $1 \leq J \leq N_{\phi^{\prime}}$, the equilibrium state $\nu_{\psi^{\prime}, J}$ associated to the potential $\psi^{\prime}$ on $\bar{X}_{J}^{\prime}$ (which is a Markov measure).

(5) If $J \in \mathcal{A}^{\prime}$ is such that $[J] \cap\left(\bigcup_{K=1}^{N_{\phi^{\prime}}} \bar{X}_{K}^{\prime}\right)=\emptyset$, then $\alpha_{J}=0$, otherwise $\alpha_{J}=\nu_{\psi^{\prime}, K}[J] \alpha_{K}^{\prime}$, where $K \leq N_{\phi^{\prime}}$ is such that $[J] \cap \bar{X}_{K}^{\prime} \neq \emptyset$, and $\alpha_{K}^{\prime} \geq 0$ is computed following (1) to (5) using $X^{\prime}$ instead of $X^{\prime}, \phi^{\prime}$ instead of $\phi$ and $\psi^{\prime}$ instead of $\psi$.

\subsection{Comparison with previous results.}

Let us compare our result with those in $[3,9]$. In [3], it is proved that the limit exists but it is not identified. In [9], the limit is proved to exist by a somewhat indirect argument and the coefficients of the barycentric decomposition of the limiting measure are not explicitly identified. Here we directly prove the existence of the limit and identify it precisely. Besides the fact that we make explicit calculations, the main idea is renormalization. The reader can see our method at work in Section 6. Finally, we are able to control the weak-star distance between $\mu_{\beta \phi}$ and its limit.

\section{The Renormalization Lemma: Proof of Theorem 1}

The following lemma is the crux of our approach. 
Renormalization Lemma. Let $\mathcal{A}$ be a finite alphabet and $X \subset \mathcal{A}^{\mathbb{Z}}$ a transitive topological Markov chain defined by an arrow set $\mathcal{E}$. Let $\phi, \psi: \mathcal{E} \rightarrow \mathbb{R}$ be 2 -symbol potentials and $\bar{X}:=\bigsqcup_{J=1}^{N} \bar{X}_{J}$ the $\phi$-maximizing SFT which decomposes into transitive components $\bar{X}_{J}$. Let $\phi^{\prime}, \psi^{\prime}: \mathcal{E}^{\prime} \rightarrow \mathbb{R}$ be the renormalized potentials (Definition 3), where $\mathcal{E}^{\prime}$ is the arrow set of the renormalized SFT $X^{\prime}$ (Definition 2), and $\mu_{\beta \phi^{\prime}+\psi^{\prime}}$ the equilibrium state of $\beta \phi^{\prime}+\psi^{\prime}$.

Then there exist $\delta=\delta(\mathcal{A}, \phi)$ (independent of $\psi$ ) such that for all $\beta$ large enough

$$
\mu_{\beta \phi+\psi}\left[\mathbf{b}_{0}^{n}\right]=\nu_{\psi, J}\left[\mathbf{b}_{0}^{n}\right] \mu_{\beta \phi^{\prime}+\phi^{\prime}}[J] \pm e^{-\beta \delta},
$$

for all cylinder $\left[\mathbf{b}_{0}^{n}\right]$ intersecting an heavy component (Definition 1), i.e., whenever $\left[\mathbf{b}_{0}^{n}\right] \cap \bar{X}_{J} \neq \emptyset$ for some $1 \leq J \leq N_{\phi}$.

From now on, when we say 'for $\beta$ large enough', this means that 'there exists $\beta_{0}=\beta_{0}(\mathcal{A}, \phi, \psi)$ such that for all $\beta \geq \beta_{0}$ '.

The proof of Theorem 1 consists in the recursive application of the Renormalization Lemma, which we assume true for the moment and which we shall prove in Section 7.

Step 1 (First Renormalization). We compute the $\phi$-maximizing subshift $\bar{X} \subset X$, which we decompose into its transitive components $\bar{X}:=\bigsqcup_{J=1}^{N} \bar{X}_{J}$. We order the heavy components so that they are indexed by $J=1, \ldots, N_{\phi}$. According to the Renormalization Lemma, there exists $\delta$ such that for $\beta$ large enough

$$
\mu_{\beta \phi+\psi}\left[\mathbf{b}_{0}^{n}\right]=\nu_{\psi, J}\left[\mathbf{b}_{0}^{n}\right] \mu_{\beta \phi^{\prime}+\phi^{\prime}}[J] \pm e^{-\beta \delta},
$$

whenever $\left[\mathbf{b}_{0}^{n}\right] \cap \bar{X}_{J} \neq \emptyset$ for some $1 \leq J \leq N_{\phi}$. In particular, for each $b \notin \bigsqcup_{J=1}^{N_{\phi}} \overline{\mathcal{A}}_{J}$ and $\beta$ large enough we have

$$
\begin{aligned}
\mu_{\beta \phi+\psi}[b] & \leq 1-\sum_{J=1}^{N_{\phi}} \sum_{a \in \overline{\mathcal{A}}_{J}} \mu_{\beta \phi+\psi}[a] \\
& \leq 1-\sum_{J=1}^{N_{\phi}} \mu_{\beta \phi^{\prime}+\phi^{\prime}}[J]\left(\sum_{a \in \overline{\mathcal{A}}_{J}} \nu_{\psi, J}[a]\right)+\#\left(\bigsqcup_{J=1}^{N_{\phi}} \overline{\mathcal{A}}_{J}\right) e^{-\beta \delta} \\
& \leq \# \mathcal{A} e^{-\beta \delta} .
\end{aligned}
$$

Hence, it follows, for $\beta$ large enough, that

$$
\mu_{\beta \phi+\psi}\left[\mathbf{b}_{0}^{n}\right] \leq e^{-\beta \frac{\delta}{2}}
$$

whenever $\left[\mathbf{b}_{0}^{n}\right] \cap \bar{X}_{J} \neq \emptyset$ for some $1 \leq J \leq N_{\phi}$. Indeed, such a cylinder contains at least a letter $b \notin \bigsqcup_{J=1}^{N_{\phi}} \overline{\mathcal{A}}_{J}$. By shift-invariance, we can assume that $\mathbf{b}_{0}=b$ and we have $\mu_{\beta \phi+\psi}\left[\mathbf{b}_{0}^{n}\right] \leq \mu_{\beta \phi+\psi}[b]$.

Step 2 (Recursion). Now, in order to compute $\mu_{\beta \phi^{\prime}+\phi^{\prime}}([J])$, we apply Step 1 to the renormalized system $\left(X^{\prime}, \beta \phi^{\prime}+\psi^{\prime}\right)$ and use (11). This yields

$$
\begin{array}{ll}
\mu_{\beta \phi^{\prime}+\psi^{\prime}}[J]=\nu_{\phi^{\prime}, K}[J] \mu_{\beta \phi^{\prime \prime}+\phi^{\prime \prime}}[K] \pm e^{-\beta \delta^{\prime}} & \text { if } J \in \overline{\mathcal{A}}_{K}^{\prime}, K \leq N_{\phi^{\prime}}, \\
\mu_{\beta \phi^{\prime}+\psi^{\prime}}[J] \leq e^{-\beta \frac{\delta^{\prime}}{2}} & \text { if } J \notin \bigsqcup_{K=1}^{N_{\phi^{\prime}}} \overline{\mathcal{A}}_{J}^{\prime},
\end{array}
$$

for $\beta$ large enough.

In particular, $\nu_{\psi^{\prime}, K}[J]$ is the first term of the factorization of $\alpha_{J}$. 
When we iterate this procedure $i$ times, we obtain a sequence of renormalized systems, the cumulative error term, and the first $i$ terms of the factorization of $\alpha_{J}$, for each $1 \leq J \leq N_{\phi}$.

Final Step (Convergence of the Recursion). Suppose we have done $i$ times the renormalization, so that we have a renormalized system $\left(X^{(i)}, \beta \phi^{(i)}+\psi^{(i)}\right)$ with a corresponding $\delta^{(i)}$. Then there are two cases: either $\# \mathcal{A}^{(i)}<\# \mathcal{A}^{(i-1)}$, or $\# \mathcal{A}^{(i)}=\# \mathcal{A}^{(i-1)}$.

In the second case, we have a maximizing SFT made only of fixed points, i.e., each symbol of the $(i-1)$-th normalization defines a heavy component. Then we necessarily have $(J, J) \in \mathcal{E}^{(i-1)}$ for each $J \in \mathcal{A}^{(i-1)}$, whence $\mathcal{E}^{(i)}=\mathcal{E}^{(i-1)} \backslash\left\{(J, J) \in \mathcal{E}^{(i-1)}\right\}$. Therefore, if we are in the second case, then $\# \mathcal{A}^{(i+1)}<\# \mathcal{A}^{(i-1)}$. The renormalization process ends because there exists some $m=m(\mathcal{A}, \phi, \psi)$ such that $\mathcal{A}^{(m)}$ is a singleton, say $\{1\}$, and we necessarily have $\mu_{\beta \phi^{(m)}+\psi^{(m)}}[1]=1$. Therefore, for $\beta$ large enough, we end up with

$$
\mu_{\beta \phi+\psi}\left[\mathbf{b}_{0}^{n}\right]=\alpha_{J} \nu_{\psi, J}\left[\mathbf{b}_{0}^{n}\right] \pm\left(\sum_{j=1}^{m-1} e^{-\beta \delta^{(j)}}\right)
$$

whenever $\left[\mathbf{b}_{0}^{n}\right] \cap \bar{X}_{J} \neq \emptyset$ for some $1 \leq J \leq N_{\phi}$.

The last claim of the theorem follows from this, by taking $C:=\delta^{(m-1)} / 2$ and $\beta$ large enough. Theorem 1 is proved.

\section{EXAMPLES}

\subsection{A Basic Example.}

Let $X=A^{\mathbb{Z}}$ with $A=\{a, b, c\}$ and the following 2-symbol potential:

$$
\phi:=\left(\begin{array}{ccc}
0 & -1 & -2 \\
-1 & 0 & -2 \\
-2 & -2 & 0
\end{array}\right) .
$$

One can of course compute $\lim _{\beta \rightarrow+\infty} \mu_{\beta \phi}$ directly, as was done in [8]. For the sake of illustration of our method, let us compute it by following the algorithm described in Section 4. In this case $\bar{X}=\operatorname{Per}_{1}(X):=\{\mathbf{a}, \mathbf{b}, \mathbf{c}\}$, with $\mathbf{a}, \mathbf{b}, \mathbf{c}$ such that $\mathbf{a}_{n}=a, \mathbf{b}_{n}=b, \mathbf{c}_{n}=c$, for all $n \in \mathbb{Z}$. Hence

$$
\lim _{\beta \rightarrow+\infty} \mu_{\beta \phi}=\alpha_{1} \delta_{\mathbf{a}}+\alpha_{2} \delta_{\mathbf{b}}+\alpha_{3} \delta_{\mathbf{c}}
$$

where $\delta_{\mathbf{x}}$ denotes the Dirac measure at $\mathbf{x}$. Notice that $\bar{\phi}=0$. Since $\psi \equiv 0$ and $\bar{X}$ is a finite union of periodic points, we have $P(\psi \mid \bar{X})=h_{\text {top }}(\bar{X})=0$. Hence potentials are already normalized. The renormalized alphabet is $A^{\prime}=\{1,2,3\}$, and the renormalized system is the topological Markov chain $X^{\prime} \subset A^{\prime} \mathbb{Z}$ described by the digraph $\left(A^{\prime}, \mathcal{E}^{\prime}\right)$ shown in the following picture: 


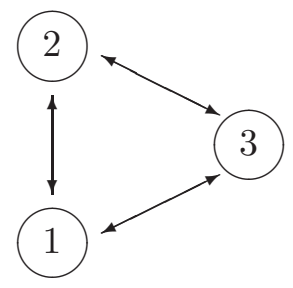

In this case, the renormalized potentials $\phi^{\prime}, \psi^{\prime}: \mathcal{E}^{\prime} \rightarrow \mathbb{R}$ are given by

$$
\phi^{\prime}:=\left(\begin{array}{ccc}
-\infty & -1 & -2 \\
-1 & -\infty & -2 \\
-2 & -2 & -\infty
\end{array}\right)
$$

and

$$
\psi^{\prime}:=\left(\begin{array}{ccc}
-\infty & 0 & 0 \\
0 & -\infty & 0 \\
0 & 0 & -\infty
\end{array}\right) .
$$

(By ' $-\infty$ ' we mean that there is no arrow.) The maximizing topological Markov chain $\bar{X}^{\prime}$ reduces to the periodic orbit of $\mathbf{x}:=(\ldots 1212 \ldots)$, which is the only heavy component, and carries only one shift-invariant measure, namely $\frac{1}{2}\left(\delta_{\mathbf{x}}+\delta_{\sigma \mathbf{x}}\right)$. Hence $\alpha_{1}=\alpha_{2}=\frac{1}{2}$ and $\alpha_{3}=0$ and

$$
\lim _{\beta \rightarrow+\infty} \mu_{\beta \phi}=\frac{1}{2}\left(\delta_{\mathbf{a}}+\delta_{\sigma \mathbf{b}}\right)
$$

\subsection{An Example with an Irrational Barycenter.}

Let $X=A^{\mathbb{Z}}$ with $A=\{a, b, c, d\}$ and the following 2-symbol potential:

$$
\phi:=\left(\begin{array}{cccc}
0 & -1 & -1 & -2 \\
-1 & 0 & -1 & -2 \\
-1 & -1 & -1 & 0 \\
-2 & -2 & -1 & 0
\end{array}\right)
$$

In this case $\bar{X}=\operatorname{Per}_{1}(X):=\{\mathbf{a}, \mathbf{b}, \mathbf{c}, \mathbf{d}\}$, with $\mathbf{a}, \mathbf{b}, \mathbf{c}$ and $\mathbf{d}$ such that $\mathbf{a}_{n}=a, \mathbf{b}_{n}=b, \mathbf{c}_{n}=c$ and $\mathbf{d}_{n}=d$ for all $n \in \mathbb{Z}$. Hence

$$
\lim _{\beta \rightarrow+\infty} \mu_{\beta \phi}=\alpha_{1} \delta_{\mathbf{a}}+\alpha_{2} \delta_{\mathbf{b}}+\alpha_{3} \delta_{\mathbf{c}}+\alpha_{4} \delta_{\mathbf{d}}
$$

Note that potentials are already normalized. The renormalized alphabet is $A^{\prime}=\{1,2,3,4\}$, and the renormalized system is the topological Markov chain $X^{\prime} \subset A^{\prime \mathbb{Z}}$ described by the digraph $\left(A^{\prime}, \mathcal{E}^{\prime}\right)$ shown in the following picture: 


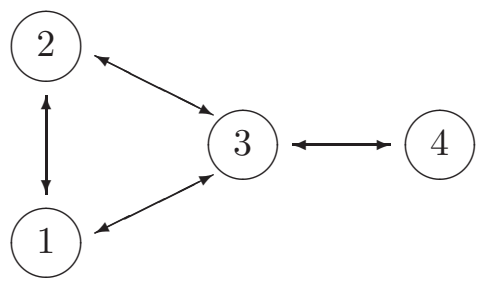

In this case, the renormalized potentials $\phi^{\prime}, \psi^{\prime}: \mathcal{E}^{\prime} \rightarrow \mathbb{R}$ are constant and such that $\phi^{\prime}(J, K)=$ -1 and $\psi^{\prime}(J, K)=0$, for each arrow $(J, K) \in \mathcal{E}^{\prime}$. The maximizing topological Markov chain $\bar{X}^{\prime}$ coincides with the renormalized topological Markov chain, therefore there is only one heavy component $\bar{X}^{\prime}=X^{\prime}$, and the second renormalization is trivial, i.e., the resulting topological Markov chain is a fixed point. From this we obtain $\alpha_{J}=\nu_{X^{\prime}}[J]$, with $\nu_{X^{\prime}}$ the measure of maximal entropy on $X^{\prime}$. It can be explicitly computed, and we finally obtain

$$
\lim _{\beta \rightarrow+\infty} \mu_{\beta \phi}=\frac{\delta_{\mathbf{a}}+\delta_{\mathbf{b}}}{2(4-\rho)}+\frac{(\rho-1)^{2} \delta_{\mathbf{c}}}{2(4-\rho)}+\frac{(\rho-1)^{2} \delta_{\mathbf{d}}}{2 \rho^{2}(4-\rho)},
$$

with $\rho=\frac{1}{3}\left(1+2 \sqrt{10} \cos \left(\frac{1}{3} \arctan (3 \sqrt{111})\right)\right)$, the largest root of the polynomial $p(x)=x^{4}-4 x^{2}-$ $2 x+1$, which is an irrational number. In the table below we present the comparison between the limiting measure and $\mu_{\beta \phi}$ for different values of the inverse temperature.

\begin{tabular}{|r|c|c|c|c|c|c|c|c|}
\hline$\beta=$ & $\log (2)$ & $2 \log (2)$ & $3 \log (2)$ & $4 \log (2)$ & $5 \log (2)$ & $6 \log (2)$ & $\cdots$ & $\infty$ \\
\hline$\mu_{\beta \phi}[a]=$ & 0.253298 & 0.259815 & 0.265413 & 0.269011 & 0.271041 & 0.272118 & $\cdots$ & 0.273237 \\
$\mu_{\beta \phi}[b]=$ & 0.253298 & 0.259815 & 0.265413 & 0.269011 & 0.271041 & 0.272118 & $\cdots$ & 0.273237 \\
$\mu_{\beta \phi}[c]=$ & 0.316672 & 0.349361 & 0.363356 & 0.369239 & 0.371810 & 0.372988 & $\cdots$ & 0.374089 \\
$\mu_{\beta \phi}[d]=$ & 0.176732 & 0.131010 & 0.105818 & 0.092738 & 0.086109 & 0.082777 & $\cdots$ & 0.079437 \\
\hline
\end{tabular}

\subsection{An Example with a Two-step Renormalization.}

Let us now take $X=A^{\mathbb{Z}}$ with $A=\{a, b, c, d, e\}$, and consider the following 2-symbol potential:

$$
\phi:=\left(\begin{array}{ccccc}
0 & -4 & -1 & -3 & -4 \\
-1 & 0 & -4 & -3 & -3 \\
-4 & -1 & 0 & -3 & -3 \\
-4 & -4 & -4 & 0 & -1 \\
-3 & -4 & -4 & -1 & 0
\end{array}\right)
$$

We have $\bar{X}=\operatorname{Per}_{1}(X):=\{\mathbf{a}, \mathbf{b}, \mathbf{c}, \mathbf{d}, \mathbf{e}\}$, with $\mathbf{a}, \mathbf{b}, \mathbf{c}, \mathbf{d}$ and $\mathbf{d}$ such that $\mathbf{a}_{n}=a, \mathbf{b}_{n}=b, \mathbf{c}_{n}=$ $c, \mathbf{d}_{n}=d$ and $\mathbf{e}_{n}=e$ for all $n \in \mathbb{Z}$, i.e,

$$
\lim _{\beta \rightarrow+\infty} \mu_{\beta \phi}=\alpha_{1} \delta_{\mathbf{a}}+\alpha_{2} \delta_{\mathbf{b}}+\alpha_{3} \delta_{\mathbf{c}}+\alpha_{4} \delta_{\mathbf{d}}+\alpha_{5} \delta_{\mathbf{e}} .
$$

As in the previous examples, potentials are already normalized. The renormalized alphabet is $A^{\prime}=\{1,2,3,4,5\}$, and the renormalized system is the topological Markov chain $X^{\prime} \subset A^{\prime} \mathbb{Z}$ described by the digraph $\left(A^{\prime}, \mathcal{E}^{\prime}\right)$ shown in the following picture. 


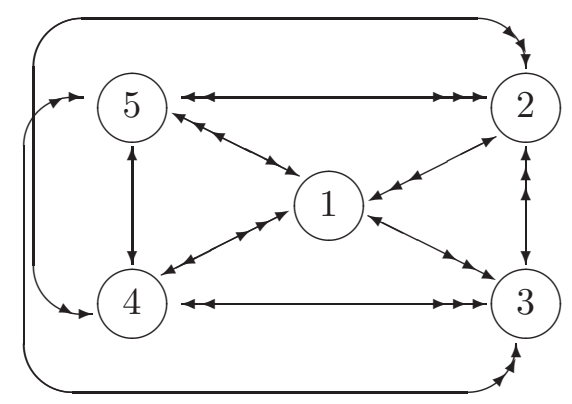

In the previous figure, we use the following convention: an arrow from $J$ to $K$ with one point means that $\phi^{\prime}(J, K)=-1$, an arrow with a double point corresponds to $\phi^{\prime}(i, j)=-2$, while an arrow with a triple point means that $\phi^{\prime}(J, K)=-3$. Since $P\left(\psi \mid \bar{X}_{J}\right)=0$ for each heavy component $1 \leq J \leq 4$, $\psi^{\prime}(J, K)=0$, for each arrow $(J, K) \in \mathcal{E}^{\prime}$. The maximizing SFT of the first renormalization $\bar{X}^{\prime}$ is composed by two heavy components as indicated in the picture below.
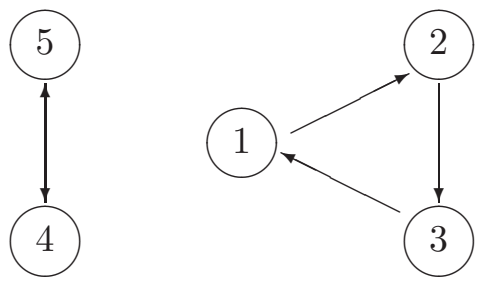

Hence, according to the algorithm, the second renormalization yields

$$
\lim _{\beta \rightarrow+\infty} \mu_{\beta \phi^{\prime}+\psi^{\prime}}=\alpha_{1}^{\prime} \frac{\delta_{\mathbf{x}}+\delta_{\sigma \mathbf{x}}}{2}+\alpha_{2}^{\prime} \frac{\delta_{\mathbf{y}}+\delta_{\sigma \mathbf{y}}+\delta_{\sigma^{2} \mathbf{y}}}{3},
$$

where $\mathbf{x}$ is the only periodic point in $\operatorname{Per}_{2}\left(X^{\prime}\right) \cap[45]$, and $\mathbf{y}$ is the only periodic point in $\operatorname{Per}_{3}\left(X^{\prime}\right) \cap$ [123]. In order to compute the coefficients $\alpha_{1}^{\prime}$ and $\alpha_{2}^{\prime}$, we need a second renormalization. The second renormalization gives the topological Markov chain $X^{\prime \prime} \subset\{1,2\}^{\mathbb{Z}}$, defined by the digraph

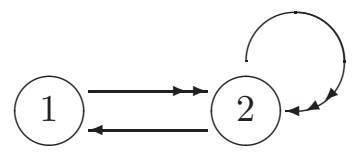

According to the algorithm, we have

$$
\phi^{\prime \prime}=\left(\begin{array}{cc}
-\infty & -1 \\
-1 & -2
\end{array}\right) \quad \psi^{\prime \prime}=\left(\begin{array}{cc}
-\infty & 0 \\
\log (5) & \log (3)
\end{array}\right)
$$


The second renormalization gives only a single heavy component, $\bar{X}^{\prime \prime}=\{\mathbf{z}, \sigma \mathbf{z}\}:=\operatorname{Per}_{2}\left(X^{\prime \prime}\right) \cap[12]$, therefore

$$
\lim _{\beta \rightarrow \infty} \mu_{\beta \phi^{\prime \prime}+\psi^{\prime \prime}}=\frac{\delta_{\mathbf{z}}+\delta_{\sigma \mathbf{z}}}{2},
$$

and we have $\alpha_{1}^{\prime}=\alpha_{2}^{\prime}=1 / 2$ for the coefficients in the limit of the first renormalization. Therefore

$$
\lim _{\beta \rightarrow+\infty} \mu_{\beta \phi}=\frac{\delta_{\mathbf{a}}+\delta_{\mathbf{b}}+\delta_{\mathbf{c}}}{6}+\frac{\delta_{\mathbf{d}}+\delta_{\mathbf{e}}}{4},
$$

for the limit of the original measure. In the table below we present the comparison between the limiting measure and $\mu_{\beta \phi}$ for different values of the inverse temperature.

\begin{tabular}{|r|c|c|c|c|c|c|c|c|}
\hline$\beta=$ & $\log (2)$ & $2 \log (2)$ & $3 \log (2)$ & $4 \log (2)$ & $5 \log (2)$ & $6 \log (2)$ & $\cdots$ & $\infty$ \\
\hline$\mu_{\beta \phi}[a]=$ & 0.19273 & 0.18423 & 0.17668 & 0.17200 & 0.16942 & 0.16807 & $\cdots$ & 0.166667 \\
\hline$\mu_{\beta \phi}[b]=$ & 0.18399 & 0.17722 & 0.17395 & 0.17115 & 0.16918 & 0.16800 & $\cdots$ & 0.166677 \\
\hline$\mu_{\beta \phi}[c]=$ & 0.19326 & 0.18343 & 0.17607 & 0.17176 & 0.16935 & 0.16805 & $\cdots$ & 0.166667 \\
\hline$\mu_{\beta \phi}[d]=$ & 0.21312 & 0.22565 & 0.23582 & 0.24227 & 0.24595 & 0.24792 & $\cdots$ & 0.250000 \\
\hline$\mu_{\beta \phi}[e]=$ & 0.21690 & 0.22946 & 0.23748 & 0.24282 & 0.24610 & 0.24796 & $\cdots$ & 0.250000 \\
\hline
\end{tabular}

\section{Proof of the Renormalization Lemma}

We assume that $\phi$ and $\psi$ are normalized as described in Subsection 3.4.

Let $E_{\phi}=\left\{\phi(C) /|C|: C \in \overline{\mathcal{C}}_{0}\right\}$, where $\overline{\mathcal{C}}_{0}$ denotes the set of all maximizing elementary circuits in $\mathcal{G}_{X}$. Clearly $E_{\phi}$ is finite and $\max E_{\phi}=\bar{\phi}=0$. We let

$$
\phi_{g}:=\max \left(E_{\phi} \backslash\{\bar{\phi}\}\right)<0
$$

denote the second largest value in $E_{\phi}$.

First Step (Factorization on Heavy Components). For each $1 \leq J \leq N_{\phi}$, let $I_{J}:=\left\{\mathbf{b} \in X: \mathbf{b}_{0} \in\right.$ $\left.\overline{\mathcal{A}}_{J}\right\}$ be the set of all points whose orbit visits the heavy component $\bar{X}_{J}$ during an interval of time containing the origin. According to Lemma 1 (proved in Section 8), we have for $\beta$ large enough

$$
\mu_{\beta \phi+\psi}\left[\mathbf{b}_{0}^{n}\right]=\nu_{\psi, J}\left[\mathbf{b}_{0}^{n}\right] \mu_{\beta \phi+\psi}\left(I_{J}\right) \pm 2 e^{\beta \frac{\phi_{g}}{4}},
$$

whenever $\left[\mathbf{b}_{0}^{n}\right] \cap \bar{X}_{J} \neq \emptyset$ for some $1 \leq J \leq N_{\phi}$.

Second Step (Excursion Markov Chain). For each a $\in X$ such that $\mathbf{a}_{0} \in \bigsqcup_{J=1}^{N_{\phi}} \overline{\mathcal{A}}_{J} \subset \overline{\mathcal{A}}$, let

$$
\begin{aligned}
i(\mathbf{a}):=\max \left\{i<0:\left(\mathbf{a}_{i-1}, \mathbf{a}_{i}\right) \notin \overline{\mathcal{E}}_{\phi}\right\}, & o(\mathbf{a}):=\min \left\{i>0: \quad\left(\mathbf{a}_{i}, \mathbf{a}_{i+1}\right) \notin \overline{\mathcal{E}}_{\phi}\right\}, \\
i^{\prime}(\mathbf{a}):=\min \{i>j(\mathbf{a}): & \left.\left(\mathbf{a}_{i-1}, \mathbf{a}_{i}\right) \in \overline{\mathcal{E}}_{\phi}\right\}, \quad o^{\prime}(\mathbf{a}):=\min \left\{i>i^{\prime}(\mathbf{a}):\left(\mathbf{a}_{i}, \mathbf{a}_{i+1}\right) \notin \overline{\mathcal{E}}_{\phi}\right\},
\end{aligned}
$$

where $\overline{\mathcal{E}}_{\phi}$ is defined in (1). Indices $i(\mathbf{a}), o(\mathbf{a}), i^{\prime}(\mathbf{a})$ and $o^{\prime}(\mathbf{a})$ are the first and second input/output times to/from heavy components of the orbit of $\mathbf{a} \in X$.

For $1 \leq J \leq N_{\phi}$ and $a, a^{\prime} \in \overline{\mathcal{A}}_{J}$, let $\left[a, a^{\prime}\right]_{J}:=\left\{\mathbf{a} \in X: \mathbf{a}_{i(\mathbf{a})}=a \in \overline{\mathcal{A}}_{J}, \mathbf{a}_{o(\mathbf{a})}=a^{\prime}\right\}$. This is the set of all points whose orbit enters the component $\bar{X}_{J}$ at vertex $a$ and leaves it at vertex $a^{\prime}$. Similarly, for $1 \leq J, K \leq N_{\phi}, a, a^{\prime} \in \bar{X}_{J}$ and $c, c^{\prime} \in \bar{X}_{K}$, let

$\left[\left[a, a^{\prime}\right]_{J},\left[c, c^{\prime}\right]_{K}\right]:=\left\{\mathbf{a} \in X: \mathbf{a}_{i(\mathbf{a})}=a \in \overline{\mathcal{A}}_{J}, \mathbf{a}_{o(\mathbf{a})}=a^{\prime} \in \overline{\mathcal{A}}_{J}, \mathbf{c}_{i^{\prime}(\mathbf{a})}=c \in \overline{\mathcal{A}}_{K}\right.$, and $\left.\mathbf{a}_{o^{\prime}(\mathbf{a})}=c^{\prime} \in \overline{\mathcal{A}}_{K}\right\}$, 
which is the set of all points entering under the shift action the component $\bar{X}_{J}$ at vertex $a$ and going out at $a^{\prime}$, and such that the next heavy component they visit is $\bar{X}_{K}$, entering at $c$ and going out at $c^{\prime}$. We clearly have

$$
\mu_{\beta \phi+\psi}\left(I_{J}\right)=\sum_{\mathbf{d}, \mathbf{e} \in \overline{\mathcal{A}}_{J}} \mu_{\beta \phi+\psi}\left(\left[a, a^{\prime}\right]_{J}\right)
$$

To estimate $\mu_{\beta \phi+\psi}\left(I_{J}\right)$, we use the Markov chain on the extended alphabet

$$
\mathcal{A}_{\text {ext }}:=\left\{\left[a, a^{\prime}\right]_{J}: 1 \leq J \leq N_{\phi}, \text { and } a, a^{\prime} \in \overline{\mathcal{A}}_{J}\right\},
$$

with transition matrix $M_{\beta \phi+\psi}: \mathcal{A}_{\text {ext }} \times \mathcal{A}_{\text {ext }} \rightarrow[0,1]$ such that

$$
M_{\beta \phi+\psi}\left(\left[a, a^{\prime}\right]_{J},\left[c, c^{\prime}\right]_{K}\right):=\frac{\mu_{\beta \phi+\psi}\left(\left[\left[a, a^{\prime}\right]_{J},\left[c, c^{\prime}\right]_{K}\right]\right)}{\mu_{\beta \phi+\psi}\left(\left[a, a^{\prime}\right]_{J}\right)} .
$$

From the shift-invariance of $\mu_{\beta \phi+\psi}$ it follows that $M_{\beta \phi+\psi}$ is a stochastic matrix. Furthermore, since $\mu_{\beta \phi+\psi}$ is ergodic, then $M_{\beta \phi+\psi}\left(\left[a, a^{\prime}\right]_{J},\left[c, c^{\prime}\right]_{K}\right)$ is irreducible and has a unique invariant distribution $\eta_{\beta \phi+\psi}$, which by construction satisfies

$$
\eta_{\beta \phi+\psi}\left(\left[a, a^{\prime}\right]_{J}\right):=\frac{\mu_{\beta \phi+\psi}\left(\left[a, a^{\prime}\right]_{J}\right)}{\sum_{K=1}^{N_{\phi}} \sum_{c, c^{\prime} \in \overline{\mathcal{A}}_{K}} \mu_{\beta \phi+\psi}\left(\left[c, c^{\prime}\right]_{K}\right)} .
$$

Third Step (Concentration on Heavy Components).

Lemma 4 states that the measure $\mu_{\beta \phi+\psi}$ concentrates on heavy the components: for $\beta$ large enough

$$
\mu_{\beta \phi+\psi}\left(\bigcup_{K=1}^{N_{\phi}} I_{K}\right) \geq 1-e^{\beta \frac{\phi_{g}}{4}}
$$

We defer its (lengthy) proof to Appendix D. Then it follows that the factor $\mu_{\beta \phi+\psi}\left(I_{J}\right)$ in (13) can be approximated by the invariant distribution $\eta_{\beta \phi+\psi}$ of the stochastic matrix $M_{\beta \phi+\psi}$. Using the fact that $\mu_{\beta \phi+\psi}\left(I_{J}\right)=\sum_{a, a^{\prime} \in \overline{\mathcal{A}}_{J}} \mu_{\beta \phi+\psi}\left(\left[a, a^{\prime}\right]_{J}\right)$ and (15) we get

$$
\mu_{\beta \phi+\psi}\left(I_{J}\right)=\sum_{K=1}^{N_{\phi}} \mu_{\beta \phi+\psi}\left(I_{K}\right) \sum_{a, a^{\prime} \in \overline{\mathcal{A}}_{J}} \eta_{\beta \phi+\psi}\left(\left[a, a^{\prime}\right]_{J}\right)
$$

from which it follows by using (16) that for $\beta$ large enough

$$
\mu_{\beta \phi+\psi}\left[\mathbf{b}_{0}^{n}\right]=\nu_{\psi, J}\left[\mathbf{b}_{0}^{n}\right] \sum_{a, a^{\prime} \in \overline{\mathcal{A}}_{J}} \eta_{\beta \phi+\psi}\left(\left[a, a^{\prime}\right]_{J}\right) \pm 3 e^{\beta \frac{\phi_{g}}{4}},
$$

whenever $\left[\mathbf{b}_{0}^{n}\right] \cap \bar{X}_{J} \neq \emptyset$ for some $1 \leq J \leq N_{\phi}$. Hence, the convergence of $\mu_{\beta \phi+\psi}$ when $\beta \rightarrow+\infty$, is controlled by the behavior of the invariant distribution $\eta_{\beta \phi+\psi}$, which we investigate now. 
Fourth Step (Excursion Potentials).

We will replace the stochastic matrix $M_{\beta \phi+\psi}$ by a transition matrix $M_{\beta \tilde{\phi}+\tilde{\psi}}$, defined by twosymbol potentials $\tilde{\phi}, \tilde{\psi}: \mathcal{A}_{\text {ext }} \rightarrow \mathbb{R}$. These excursion potentials are such that the one-marginal $\mu_{\beta \tilde{\phi}+\tilde{\psi}}^{(1)}$ of the Gibbs measure $\mu_{\beta \tilde{\phi}+\tilde{\psi}}$ approaches the invariant distribution $\eta_{\beta \phi+\psi}$ of the stochastic matrix $M_{\beta \phi+\psi}$ as $\beta \rightarrow+\infty$.

The excursion potentials are defined as follows. Let $\mathbf{v}_{\beta \phi+\psi}$ be the right maximal eigenvector of $\mathcal{M}_{\beta \phi+\psi}$, and $\tilde{\mathcal{M}}_{\beta \phi+\psi}<\mathcal{M}_{\beta \phi+\psi}$ be the submatrix of $\mathcal{M}_{\beta \phi+\psi}$ obtained by excluding all the heavy components. For each heavy component $1 \leq J \leq N_{\phi}$ and $a, a^{\prime} \in \overline{\mathcal{A}}_{J}$ let $\tilde{\mathbf{v}}_{\beta \phi+\psi}(a):=$ $\sum_{b \in \mathcal{A}} \tilde{\mathcal{M}}_{\beta \phi+\psi}(a, b) \mathbf{v}_{\beta \phi+\psi}(b)$. Then define $\tilde{\phi}, \tilde{\psi}: \mathcal{A}_{\text {ext }} \rightarrow \mathbb{R}$ such that

$$
\begin{aligned}
\tilde{\phi}\left(\left[a, a^{\prime}\right]_{J},\left[c, c^{\prime}\right]_{K}\right) & :=\overleftarrow{\phi}\left(a^{\prime}\right)+\vec{\phi}\left(a^{\prime}, c\right)-\overleftarrow{\phi}(c), \\
\left.\tilde{\psi}\left(\left[a, a^{\prime}\right]_{J},\left[c, c^{\prime}\right]_{K}\right)\right) & :=\log \left(\sum_{a^{\prime} \rightarrow c \in \overline{\operatorname{Path}}\left[a^{\prime}, c\right]} e^{\psi\left(a^{\prime} \rightarrow c\right)+P_{\psi}\left(a^{\prime} \rightarrow c\right)}\right)+\log \left(\mathbf{v}_{\psi, J}\left(a^{\prime}\right) \mathbf{w}_{\psi, K}(c)\right)
\end{aligned}
$$

where $\overline{\operatorname{Path}}\left[a^{\prime}, c\right] \subset \operatorname{Path}\left[a^{\prime}, c\right]$ is the set of all simple paths from $a^{\prime}$ to $c$ maximizing $\phi$. Here we have used the transition pressure, central term, and transition term as defined in (7),(8) and (9) respectively.

To the excursion potential we associate a transition matrix $M_{\beta \tilde{\phi}+\tilde{\psi}}: \mathcal{A}_{\text {ext }} \times \mathcal{A}_{\text {ext }} \rightarrow \mathbb{R}^{+}$such that

$$
M_{\beta \tilde{\phi}+\tilde{\psi}}\left(\left[a, a^{\prime}\right]_{J},\left[c, c^{\prime}\right]_{K}\right):=\exp \left((\beta \tilde{\phi}+\tilde{\psi})\left(\left[a, a^{\prime}\right]_{J},\left[c, c^{\prime}\right]_{K}\right)\right) .
$$

The matrices $M_{\beta \tilde{\phi}+\tilde{\psi}}$ and $M_{\beta \phi+\psi}$ can be related using Lemma 2: for $\beta$ large enough we have

$$
M_{\beta \phi+\psi}\left(\left[a, a^{\prime}\right]_{J},\left[c, c^{\prime}\right]_{K}\right)=M_{\beta \tilde{\phi}+\tilde{\psi}}\left(\left[a, a^{\prime}\right]_{J},\left[c, c^{\prime}\right]_{K}\right) \frac{e^{\beta \overleftarrow{\phi}\left(c^{\prime}\right)} \mathbf{v}_{\psi, K}\left(c^{\prime}\right) \tilde{\mathbf{v}}_{\beta \phi+\psi}\left(c^{\prime}\right)}{e^{\beta \overleftarrow{\phi}\left(a^{\prime}\right)} \mathbf{v}_{\psi, J}\left(a^{\prime}\right) \tilde{\mathbf{v}}_{\beta \phi+\psi}\left(a^{\prime}\right)} \frac{e^{ \pm e^{-\beta \delta}}}{\rho_{\beta \phi+\psi}-1},
$$

for all $1 \leq J, K \leq N_{\phi}, a, a^{\prime} \in \overline{\mathcal{A}}_{J}, c, c^{\prime} \in \overline{\mathcal{A}}_{K}$. Note that $\delta_{\phi}>\phi_{g} / 6$.

The closeness between the one-marginal $\mu_{\beta \tilde{\phi}+\tilde{\psi}}^{(1)}$ of the Gibbs measure $\mu_{\beta \tilde{\phi}+\tilde{\psi}}$ and the invariant distribution $\eta_{\beta \phi+\psi}$ of the stochastic matrix $M_{\beta \phi+\psi}$ follows from (18) after the following considerations.

First notice that the matrix $\bar{N}: \mathcal{A}_{\text {ext }} \times \mathcal{A}_{\text {ext }} \rightarrow \mathbb{R}^{+}$defined by

$$
\bar{N}\left(\left[a, a^{\prime}\right]_{J},\left[c, c^{\prime}\right]_{K}\right):=M_{\beta \tilde{\phi}+\tilde{\psi}}\left(\left[a, a^{\prime}\right]_{J},\left[c, c^{\prime}\right]_{K}\right) \frac{e^{\beta \overleftarrow{\phi}\left(c^{\prime}\right)} \mathbf{v}_{\psi, K}\left(c^{\prime}\right) \tilde{\mathbf{v}}_{\beta \phi+\psi}\left(c^{\prime}\right)}{e^{\beta \overleftarrow{\phi}\left(a^{\prime}\right)} \mathbf{v}_{\psi, J}\left(a^{\prime}\right) \tilde{\mathbf{v}}_{\beta \phi+\psi}\left(a^{\prime}\right)} \frac{1}{\rho_{\beta \phi+\psi}-1}
$$

is precisely the transition matrix associated to the potential $\Phi:=(\beta \tilde{\phi}+\tilde{\psi})+(h-\sigma \circ h)-\log \left(\rho_{\beta \phi+\psi}-\right.$ 1 ), where $h: \mathcal{A}_{\text {ext }}^{\mathbb{Z}} \rightarrow \mathbb{R}$ is given by

$$
h\left(\left[a_{0}, a_{0}^{\prime}\right]_{J_{0}}\left[a_{1}, a_{1}^{\prime}\right]_{J_{1}} \cdots\right)=-\beta \overleftarrow{\phi}\left(a_{0}^{\prime}\right)-\log \left(\mathbf{v}_{\psi, J_{0}}\right)-\log \left(\tilde{\mathbf{v}}_{\beta \phi+\psi}\right)\left(a_{0}^{\prime}\right)
$$

The potentials $\beta \tilde{\phi}+\tilde{\psi}$ and $(\beta \tilde{\phi}+\tilde{\psi})+(h-\sigma \circ h)$ are cohomologous, so they define exactly the same Gibbs state (see [2] for details). Furthermore, the potentials $(\beta \tilde{\phi}+\tilde{\psi})+(h-\sigma \circ h)$ and $\Phi$ differ only by a constant term, therefore they define the same Gibbs state as well. 
The one-marginal of the Gibbs measure $\mu_{\Phi}$, which coincides with the one-marginal of $\mu_{\beta \tilde{\phi}+\tilde{\psi}}$, is completely determined by the maximal eigensystem of the matrix $\bar{N}$. Indeed, the analogous of (3) holds, and we have

$$
\nu_{\Phi}\left(\left[a, a^{\prime}\right]_{J}\right)=\mathbf{v}_{\bar{N}}\left(\left[a, a^{\prime}\right]_{J}\right) \mathbf{w}_{\bar{N}}\left(\left[a, a^{\prime}\right]_{J}\right)
$$

for each $\left[a, a^{\prime}\right]_{J} \in \mathcal{A}_{\text {ext }}$. Here $\mathbf{v}_{\bar{N}}$ and $\mathbf{w}_{\bar{N}}$ are respectively the right and left eigenvectors of $\bar{N}$, associated to the maximal eigenvalue $\rho_{\bar{N}}:=\max (\operatorname{spec}(\bar{N}))$, and normalized such that $\mathbf{w}_{\bar{N}}^{\dagger} \mathbf{v}_{\bar{N}}=1$.

Now, we claim that $\eta_{\beta \phi+\psi}=\nu_{\Phi} e^{ \pm 8\left(\# \mathcal{A}_{\text {ext }}-1\right) e^{-\beta \delta}}$. By the above cohomological arguments we also have $\eta_{\beta \phi+\psi}=\mu_{\beta \tilde{\phi}+\tilde{\psi}}^{(1)} e^{ \pm 8\left(\# \mathcal{A}_{\text {ext }}-1\right) e^{-\beta \delta}}$. The claim follows from the application of Proposition 5 to $M_{\beta \phi+\psi}$ and $\bar{N}$ which are proved to be projectively close by Lemma 2 . Therefore, taking into account (17), it follows that for $\beta$ large enough

$$
\mu_{\beta \phi+\psi}\left[\mathbf{b}_{0}^{n}\right]=\nu_{\psi, J}\left[\mathbf{b}_{0}^{n}\right] \sum_{a, a^{\prime} \in \overline{\mathcal{A}}_{J}} \mu_{\beta \tilde{\phi}+\tilde{\psi}}^{(1)}\left(\left[a, a^{\prime}\right]_{J}\right) \pm 9\left(\# \mathcal{A}_{\mathrm{ext}}-1\right) e^{-\beta \delta},
$$

whenever $\left[\mathbf{b}_{0}^{n}\right] \cap \bar{X}_{J} \neq \emptyset$ for some $1 \leq J \leq N_{\phi}$.

Last Step (The Projection and the Renormalized Potentials).

Let us now simplify the expression for $\mu_{\beta \phi+\psi}\left[\mathbf{b}_{0}^{n}\right]$ we just proved by first making a dimensional reductionn (projection of the alphabet), followed by a simplification of the resulting potential, which will allow us to define the renormalized system.

Let $\mathcal{A}^{\prime}=\left\{1,2, \ldots, N_{\phi}\right\}$ be set of indices of heavy components. Define the projection $\pi: \mathcal{A}_{\text {ext }} \rightarrow \mathcal{A}^{\prime}$ such that $\pi\left(\left[a, a^{\prime}\right]_{J}\right)=J$ for all $1 \leq J \leq N_{\phi}$ and $a, a^{\prime} \in \overline{\mathcal{A}}_{J}$, and extend it coordinatewise to $\left(\mathcal{A}_{\text {ext }}\right)^{\mathbb{Z}}$. Let $\bar{\mu}:=\mu_{\beta \tilde{\phi}+\tilde{\psi}} \circ \pi^{-1}$ denote the pull back of the measure $\mu_{\beta \tilde{\phi}+\tilde{\psi}}$ under the projection $\pi$. Since

$$
\bar{\mu}[J]:=\sum_{a, a^{\prime} \in \overline{\mathcal{A}}_{J}} \mu_{\beta \tilde{\phi}+\tilde{\psi}}\left(\left[a, a^{\prime}\right]_{J}\right) \equiv \sum_{a, a^{\prime} \in \overline{\mathcal{A}}_{J}} \mu_{\beta \tilde{\phi}+\tilde{\psi}}^{(1)}\left(\left[a, a^{\prime}\right]_{J}\right),
$$

for each $J \in \mathcal{A}^{\prime}$ and $a, a^{\prime} \in \overline{\mathcal{A}}_{J}$, then (19) can be writen, for $\beta$ large enough, as

$$
\mu_{\beta \phi+\psi}\left[\mathbf{b}_{0}^{n}\right]=\nu_{\psi, J}\left[\mathbf{b}_{0}^{n}\right] \bar{\mu}[J] \pm 9\left(\# \mathcal{A}_{\text {ext }}-1\right) e^{-\beta \delta},
$$

whenever $\left[\mathbf{b}_{0}^{n}\right] \cap \bar{X}_{J} \neq \emptyset$ for some $1 \leq J \leq N_{\phi}$. In Lemma 3 we prove that $\bar{\mu}$ equals the Gibbs measure (more properly called Parry measure) $\mu_{\bar{\Phi}}$ defined by the 2 -symbol potential $\bar{\Phi}: \mathcal{A}_{\text {ext }} \times \mathcal{A}_{\text {ext }} \rightarrow \mathbb{R}$ such that

$$
\bar{\Phi}(J, K)=\log \sum_{a^{\prime} \in \overline{\mathcal{A}}_{J}, c \in \overline{\mathcal{A}}_{K}} \exp \left((\beta \tilde{\phi}+\tilde{\psi})\left(\left[a, a^{\prime}\right]_{J},\left[c, c^{\prime}\right]_{K}\right)\right)
$$

Hence, in order to compute $\bar{\mu}_{\beta \tilde{\phi}+\tilde{\psi}}(J) \equiv \mu_{\bar{\Phi}}(J)$, we only have to find the left and right positive eigenvectors, $\mathbf{w}_{\bar{\Phi}}$ and $\mathbf{v}_{\bar{\Phi}}$, associated to the maximal eigenvalue $\rho_{\bar{\Phi}}$ of the transition matrix $\mathcal{M}_{\bar{\Phi}}$ : $\mathcal{A}^{\prime} \times \mathcal{A}^{\prime} \rightarrow \mathbb{R}^{+}$given by

$$
\mathcal{M}_{\bar{\Phi}}(J, K):=\sum_{a^{\prime} \in \overline{\mathcal{A}}_{J}, c \in \overline{\mathcal{A}}_{K}} \exp \left(\beta \tilde{\phi}\left(a^{\prime}, c\right)+\tilde{\psi}\left(a^{\prime}, c\right)\right) .
$$

Instead of computing the left and right positive eigenvectors of $\overline{\mathcal{M}}_{\beta \tilde{\phi}+\tilde{\psi}}$, let us first approximate this matrix by a more convenient one. Let us recall the definition of the renormalized potentials 
(see (10) above). For $1 \leq J, K \leq N_{\phi}$, let

$$
\begin{aligned}
\phi^{\prime}(J, K) & :=\max \left\{\tilde{\phi}\left(a^{\prime}, c\right): a^{\prime} \in \overline{\mathcal{A}}_{J}, c \in \overline{\mathcal{A}}_{K}\right\}, \\
\psi^{\prime}(J, K) & :=\log \sum_{\left(a^{\prime}, c\right) \in \overline{\mathcal{A}}_{J, K}} e^{\tilde{\psi}\left(a^{\prime}, c\right)}
\end{aligned}
$$

where $\overline{\mathcal{A}}_{J, K}:=\left\{\left(a^{\prime}, c\right) \in \overline{\mathcal{A}}_{J} \times \overline{\mathcal{A}}_{K}: \tilde{\phi}\left(a^{\prime}, c\right)=\phi^{\prime}(J, K)\right\}$. With this renormalized potentials, a rather direct computation allows us to write, for $\beta$ large enough,

$$
\mathcal{M}_{\bar{\Phi}}=\mathcal{M}_{\beta \phi^{\prime}+\psi^{\prime}} \exp \left( \pm e^{-\beta \tilde{\delta}}\right)
$$

with

$$
\tilde{\delta}:=\frac{1}{2} \max _{J, K \in \mathcal{A}^{\prime}}\left(\phi^{\prime}(J, K)-\max \left\{\tilde{\phi}\left(a^{\prime}, c\right) \in \overline{\mathcal{A}}_{J} \times \overline{\mathcal{A}}_{K} \backslash \overline{\mathcal{A}}_{J, K}\right\}\right) .
$$

Here $\mathcal{M}_{\beta \phi^{\prime}+\psi^{\prime}}$ is defined in the same way as $\mathcal{M}_{\beta \phi+\psi}$, using the renormalized potentials just defined. Now, according to Proposition 5, the invariant distribution $\eta_{\beta \phi^{\prime}+\psi^{\prime}}$ is close to the one marginal of $\bar{\mu}_{\bar{\Phi}}$. We have that $\nu_{\bar{\Phi}}=\eta_{\beta \phi^{\prime}+\psi^{\prime}} \exp \left( \pm 8\left(\# \mathcal{A}^{\prime}-1\right) e^{-\beta \tilde{\delta}}\right)$, and from this it follows that for $\beta$ large enough

$$
\begin{aligned}
\mu_{\beta \phi+\psi}\left[\mathbf{b}_{0}^{n}\right] & =\nu_{\psi, J}\left[\mathbf{b}_{0}^{n}\right] \eta_{\beta \phi^{\prime}+\psi^{\prime}}(J) \pm 18\left(\# \mathcal{A}_{\text {ext }}-1\right) e^{-\beta \tilde{\delta}} \\
& =\nu_{\psi, J}\left[\mathbf{b}_{0}^{n}\right] \mu_{\beta \phi^{\prime}+\psi^{\prime}}([J]) \pm 18\left(\# \mathcal{A}_{\text {ext }}-1\right) e^{-\beta \tilde{\delta}}
\end{aligned}
$$

whenever $\left[\mathbf{b}_{0}^{n}\right] \cap \bar{X}_{J} \neq \emptyset$ for some $1 \leq J \leq N_{\phi}$. The renormalization Lemma follows by taking $\delta^{\prime}:=\min \left(\frac{\tilde{\delta}}{2}, \delta\right)$ and for large enough $\beta$.

\section{Auxiliary Lemmas}

We devote this section to statements and proofs of the auxiliary Lemmas used in the proof of the Renormalization Lemma (Section 7). We start with the more technical one, Lemma 1, which we prove by using periodic approximations of the Gibbs measure $\mu_{\beta \phi+\psi}$. We are able to give precise estimates of the speed of convergence of these periodic approximations, based on a refined version of the Perron-Frobenius Theorem which we present in Appendix A.

\subsection{Periodic Approximations and Factorization on Heavy Components.}

\subsubsection{Transition Matrices and Periodic Approximations.}

The matrix $\mathcal{M}_{\beta \phi+\psi}$ defined in (2) is irreducible and periodic. If we let $\bar{p}$ be its period, this means that there exists a partition $\left\{\mathcal{A}_{i}, 0 \leq i \leq \bar{p}\right\}$ of $\mathcal{A}\left(\mathcal{A}=\bigsqcup_{i=0}^{\bar{p}-1} \mathcal{A}_{i}\right)$, and nonnegative rectangular 
matrices $\mathcal{Q}_{\beta \phi+\psi, i}: \mathcal{A}_{i+1} \times \mathcal{A}_{i} \rightarrow \mathbb{R}^{+}$, (indices are taken mod $\bar{p}$ ), such that

$$
\mathcal{M}_{\beta \phi+\psi}=\left(\begin{array}{ccccc}
0 & \mathcal{Q}_{\beta \phi+\psi, 1} & 0 & \cdots & 0 \\
0 & 0 & \mathcal{Q}_{\beta \phi+\psi, 2} & \cdots & 0 \\
0 & 0 & 0 & \cdots & 0 \\
\vdots & \vdots & \vdots & \ddots & \vdots \\
0 & 0 & 0 & \cdots & \mathcal{Q}_{\beta \phi+\psi, \bar{p}-1} \\
\mathcal{Q}_{\beta \phi+\psi, 0} & 0 & 0 & \cdots & 0
\end{array}\right) .
$$

We also have

$$
\mathcal{M}_{\beta \phi+\psi}^{\bar{p}}=\left(\begin{array}{ccccc}
\mathcal{M}_{\beta \phi+\psi, 0} & 0 & 0 & \cdots & 0 \\
0 & \mathcal{M}_{\beta \phi+\psi, 1} & 0 & \cdots & 0 \\
0 & 0 & \mathcal{M}_{\beta \phi+\psi, 2} & \cdots & 0 \\
\vdots & \vdots & \vdots & \ddots & \vdots \\
0 & 0 & 0 & \cdots & \mathcal{M}_{\beta \phi+\psi, \bar{p}-1}
\end{array}\right)
$$

with $\mathcal{M}_{\beta \phi+\psi, i}: \mathcal{A}_{i} \times \mathcal{A}_{i} \rightarrow \mathbb{R}^{+}$primitive for each $0 \leq i<\bar{p}$. Therefore, according to PerronFrobenius Theorem (Theorem 2 in Appendix A) there are unique left and right maximal eigenvectors $\mathbf{v}_{\beta \phi+\psi, i}>0$ and $\mathbf{w}_{\beta \phi+\psi, i}>0$, associated to the maximal eigenvalue $\max \left|\operatorname{spec}\left(\mathcal{M}_{\beta \phi+\psi, i}\right)\right|=$ $\rho_{\beta \phi+\psi}^{\bar{p}}$, and normalized such that $\mathbf{w}_{\beta \phi+\psi, i}^{\dagger} \mathbf{v}_{\beta \phi+\psi, i}=1$. With this we define $\mathbf{v}_{\beta \phi+\psi}:=1 / \sqrt{\bar{p}} \otimes_{i=0}^{\bar{p}-1}$ $\mathbf{v}_{\beta \phi+\psi, i}$, and similarly for $\mathbf{w}_{\beta \phi+\psi}$. The vectors $\mathbf{v}_{\beta \phi+\psi}$ and $\mathbf{w}_{\beta \phi+\psi}$ so defined are the unique left and right eigenvectors associate to the maximal eigenvalue $\rho_{\beta \phi+\psi}:=\max \left|\operatorname{spec}\left(\mathcal{M}_{\beta \phi+\psi}\right)\right|$, normalized such that $\mathbf{w}_{\beta \phi+\psi}^{\dagger} \mathbf{v}_{\beta \phi+\psi}=1$.

For each $\mathbf{b}_{0}^{n} \in \mathcal{A}^{n+1}$ and $p=k \bar{p}>n$ we define the period $-p$ approximation to $\mu_{\beta \phi+\psi}\left[\mathbf{b}_{0}^{n}\right]$ by

$$
\begin{aligned}
\mathcal{P}_{\beta \phi+\psi}^{(p)}\left[\mathbf{b}_{0}^{n}\right] & :=\frac{\sum_{\mathbf{a} \in \operatorname{Per}_{p}(X) \cap\left[\mathbf{b}_{0}^{n}\right]} e^{S_{p}(\beta \phi+\psi)(\mathbf{a})}}{\sum_{\mathbf{a} \in \operatorname{Per}_{p}(X)} e^{\beta S_{p}(\beta \phi+\psi)(\mathbf{a})}} \\
& \equiv \frac{\prod_{i=0}^{n-1} \mathcal{M}_{\beta \phi+\psi}\left(\mathbf{b}_{i}, \mathbf{b}_{i+1}\right) \mathcal{M}_{\beta \phi+\psi}^{p-n}\left(\mathbf{b}_{n}, \mathbf{b}_{0}\right)}{\operatorname{tr}\left(\mathcal{M}_{\beta \phi+\psi}^{p}\right)}
\end{aligned}
$$

with $S_{p}(\beta \phi+\psi)(\mathbf{a}):=\beta \sum_{i=0}^{p-1} \phi\left(\mathbf{a}_{i}, \mathbf{a}_{i+1}\right)+\sum_{i=0}^{p-1} \psi\left(\mathbf{a}_{i}, \mathbf{a}_{i+1}\right)$ for each $\mathbf{b} \in X$.

The following result provides an estimate of the convergence rate of $\mathcal{P}_{\beta \phi+\psi}^{(p)}$ towards $\mu_{\beta \phi+\psi}$ when $p \rightarrow \infty$, as a function of $\beta$ and the potential $\phi$. The proof is deferred to Appendix A.

Proposition 1 (Periodic Approximation). Let $\mu_{\beta \phi+\psi}$ be the unique equilibrium state of $\beta \phi+\psi$ defined in (3) and let $\ell$ be an upper bound for the primitivity indices of the matrices $\mathcal{M}_{\beta \phi+\psi, i}$, $0 \leq i \leq \bar{p}$. Then, for $\gamma>s_{\phi}:=2 \bar{p} \ell\|\phi\|_{\infty}$ and $\beta$ large enough, we have

$$
\mu_{\beta \phi+\psi}\left[\mathbf{b}_{0}^{n}\right]=\mathcal{P}_{\beta \phi+\psi}^{(k \bar{p})}\left[\mathbf{b}_{0}^{n}\right] \exp \left( \pm e^{-\beta\left(\gamma-s_{\phi}\right)}\right),
$$

for all $\left[\mathbf{b}_{0}^{n}\right]$ such that $\left[\mathbf{b}_{0}^{n}\right] \cap X \neq \emptyset$. such that $k \bar{p}>e^{\beta \gamma}+n$. 
Let us recall that for each $1 \leq J \leq N, \overline{\mathcal{A}}_{J} \subset \mathcal{A}$ denote the vertex set of the digraph $\mathcal{G}_{J}:=\left(\overline{\mathcal{A}}_{J}, \overline{\mathcal{E}}_{J}\right)$ associated to the transitive component $\bar{X}_{J} \subset \bar{X}$. Let us define the matrices $\mathcal{M}_{\beta \phi+\psi, J}: \overline{\mathcal{A}}_{J} \times \overline{\mathcal{A}}_{J} \rightarrow$ $\mathbb{R}^{+}$such that

$$
\mathcal{M}_{\beta \phi+\psi, J}\left(a,^{\prime}\right)= \begin{cases}e^{(\beta \phi+\psi)\left(a, a^{\prime}\right)} & \text { if }\left(a, a^{\prime}\right) \in \overline{\mathcal{E}}_{J} \\ 0 & \text { otherwise }\end{cases}
$$

Now, for each $1 \leq J \leq N$ and $a, a^{\prime} \in \overline{\mathcal{A}}_{J}$ let $a \rightsquigarrow a^{\prime}$ be any path in the digraph $\mathcal{G}_{J}$, going from $a$ to $a^{\prime}$. Since any circuit $C$ in $\mathcal{G}_{J}$ is such that $\phi(C):=|C| \bar{\phi}=0$, then the sum $\phi\left(a \rightsquigarrow a^{\prime}\right)$ does not depend on the chosen path $a \rightsquigarrow a^{\prime}$. Therefore for each $1 \leq J \leq N$ and $a, a^{\prime} \in \overline{\mathcal{A}}_{J}$, we can define the $\left(a, a^{\prime}\right)$-compensation term,

$$
\overleftarrow{\phi}\left(a, a^{\prime}\right):=\phi\left(a \rightsquigarrow a^{\prime}\right) \text {, with } a \rightsquigarrow a^{\prime} \text { a path in } \mathcal{G}_{J} \text { from } a^{\prime} \text { to } a
$$

which relates products of the matrix $\mathcal{M}_{\psi, J}$ to products of the matrix $\mathcal{M}_{\beta \phi+\psi, J}$ defined in (4). Indeed, since we have fixed $\bar{\phi}=0$, it readily follows that

$$
\prod_{i=0}^{n} \mathcal{M}_{\beta \phi+\psi, J}^{k}\left(\mathbf{a}_{i}, \mathbf{a}_{i+1}\right)=e^{\beta \overleftarrow{\phi}\left(\mathbf{a}_{0}, \mathbf{a}_{n}\right)} \prod_{i=0}^{n} \mathcal{M}_{\psi, J}^{k}\left(\mathbf{a}_{i}, \mathbf{a}_{i+1}\right)
$$

for each $1 \leq J \leq N$ and $\mathbf{a} \in X$ such that $\left[\mathbf{a}_{0}^{n}\right] \cap \bar{X}_{J} \neq 0$.

Now, for each $1 \leq J \leq N$ the transition matrix $\mathcal{M}_{\psi, J}$ is irreducible, therefore there exists a partition $\overline{\mathcal{A}}_{J}:=\bigsqcup_{i=0}^{p_{J}-1} \overline{\mathcal{A}}_{J, i}$, such that

$$
\mathcal{M}_{\psi, J}=\left(\begin{array}{ccccc}
0 & \mathcal{Q}_{J, 1} & 0 & \cdots & 0 \\
0 & 0 & \mathcal{Q}_{J, 2} & \cdots & 0 \\
0 & 0 & 0 & \cdots & 0 \\
\vdots & \vdots & \vdots & \ddots & 0 \\
0 & 0 & 0 & \cdots & \mathcal{Q}_{J, p_{J}-1} \\
\mathcal{Q}_{J, 0} & 0 & 0 & \cdots & 0
\end{array}\right)
$$

The rectangular matrices $\mathcal{Q}_{J, i}: \overline{\mathcal{A}}_{J, i+1} \times \overline{\mathcal{A}}_{J, i} \rightarrow \mathbb{R}^{+}$, (indices are taken $\bmod p_{J}$ ), are non-negative, and such that

$$
\mathcal{M}_{\psi, J}^{p_{J}}=\left(\begin{array}{ccccc}
\mathcal{M}_{J, 0} & 0 & 0 & \cdots & 0 \\
0 & \mathcal{M}_{J, 1} & 0 & \cdots & 0 \\
0 & 0 & \mathcal{M}_{J, 2} & \cdots & 0 \\
\vdots & \vdots & \vdots & \ddots & 0 \\
0 & 0 & 0 & \cdots & \mathcal{M}_{J, p_{J}-1}
\end{array}\right)
$$

with $\mathcal{M}_{J, i}: \overline{\mathcal{A}}_{J, i} \times \overline{\mathcal{A}}_{J, i} \rightarrow \mathbb{R}^{+}$primitive for each $0 \leq i<p_{J}$.

Once again, Perron-Frobenius Theorem ensures that, for each $0 \leq j<p_{J}$, there are unique left and right maximal eigenvectors $\mathbf{v}_{J, i}>0$ and $\mathbf{w}_{J, i}>0$, associated to the maximal eigenvalue $\rho_{\psi, J}^{p_{J}}:=\max \left|\operatorname{spec}\left(\mathcal{M}_{J, i}\right)\right|$, satisfying $\mathbf{w}_{J, i}^{\dagger} \mathbf{v}_{J, i}=1$. With this we define $\mathbf{v}_{\psi, J}, \mathbf{w}_{\psi, J}: \overline{\mathcal{A}}_{J} \rightarrow \mathbb{R}^{+}$ such that $\mathbf{v}_{\psi, J}(\mathbf{f})=\left(\sqrt{p_{J}}\right) \mathbf{v}_{J, i}(a)$ for $a \in \overline{\mathcal{A}}_{J, i}$ and similarly for $\mathbf{w}_{\psi, J}$, which are the left and right eigenvectors associated to $\rho_{\psi, J}:=\max \left|\operatorname{spec}\left(\mathcal{M}_{\psi, J}\right)\right|$, normalized such that $\mathbf{w}_{\psi, J}^{\dagger} \mathbf{v}_{\psi, J}=1$. 
Corollary 1 in Appendix A ensures the existence of constants $C_{\psi}>0$ and $0 \leq \tau_{\psi}<1$ such that

$$
\mathcal{M}_{\psi, J}^{k p_{J}+r}\left(a, a^{\prime}\right)=\frac{\mathbf{v}_{\psi, J}(a) \mathbf{w}_{\psi, J}\left(a^{\prime}\right)}{\rho_{\psi, J}^{k p_{J}+r}} e^{ \pm C_{\psi} \tau_{\psi}^{k}}
$$

for each transitive component $1 \leq J \leq N$, and each $a, a^{\prime} \in \overline{\mathcal{A}}_{J}$ such that $a \in \overline{\mathcal{A}}_{J, i}$ and $a^{\prime} \in \overline{\mathcal{A}}_{J,(i+r)}$ (indices taken $\bmod p_{J}$ ).

As mentioned above, to each transitive component $\bar{X}_{J} \subset \bar{X}$ we can associate the Markovian measure $\nu_{\psi, J}$ defined by (5), which is precisely the equilibrium state on $\bar{X}_{J}$ associate to the potential $\psi \mid \bar{X}_{J}$. Because of the normalization $P(\psi \mid \bar{X})=0$, we have $\rho_{\psi, J} \leq 1$ for each $1 \leq J \leq N$, and $\rho_{\psi, J}=1$ for the heavy components $1 \leq J \leq N_{\phi}$.

Notice that the transition matrices $\mathcal{M}_{\psi, J}$ depend only on the potential $\psi$, so that they not change with the inverse temperature $\beta$.

\subsubsection{Approximating the Measure of Incursions.}

The Renormalization Lemma involves the system of incursions into heavy components. It turns out that the measure $\mu_{\beta \phi+\psi}\left[\mathbf{b}_{0}^{n}\right]$ of cylinders $\left[\mathbf{b}_{0}^{n}\right]$ intersecting a heavy component $\bar{X}_{J}$ is almost proportional to its $\nu_{\psi, J}$ measure (Lemma 1 below). This is what we call the approximated measure for incursions.

Fix $q<p=k \bar{p}$, and let

$$
X^{(p, q)}:=\left\{\mathbf{a} \in \operatorname{Per}_{p}(X):\left[\mathbf{a}_{p-q}^{q-1}\right] \cap \bar{X} \neq \emptyset\right\} .
$$

A periodic point $\mathbf{a} \in X^{(p, q)}$ is such that its suffix-prefix factor $\mathbf{a}_{p-q} \mathbf{a}_{p-q+1} \cdots \mathbf{a}_{0} \mathbf{a}_{1} \cdots \mathbf{a}_{q-1}$ defines a path in $\mathcal{G}_{X}$ composed by maximizing elementary circuits, therefore

$$
S_{2 q} \phi\left(\sigma^{-q}(\mathbf{a})\right) \geq-\# \mathcal{A}\|\phi\|_{\infty},
$$

for each $\mathbf{a} \in X^{(p, q)}$.

Let us recall that each heavy component $\bar{X}_{J} \subset \bar{X}$ has associated the Markovian measure $\nu_{\psi, J}$ given by

$$
\nu_{\psi, J}\left[\mathbf{b}_{0}^{n}\right]:=\mathbf{w}_{J}\left(\mathbf{b}_{0}\right) \prod_{i=0}^{n-1} \mathcal{M}_{\psi, J}\left(\mathbf{b}_{i}, \mathbf{b}_{i+1}\right) \mathbf{v}_{\psi, J}\left(\mathbf{b}_{n}\right),
$$

for each $\mathbf{b} \in X$ such that $\left[\mathbf{b}_{0}^{n}\right] \cap \bar{X}_{J} \neq \emptyset$.

Now, for each $1 \leq J \leq N_{\phi}$ and $p=k \bar{p}$ with $k \in \mathbb{N}$, let

$$
I_{J}^{(p)}:=\left\{\mathbf{b} \in \operatorname{Per}_{p}(X): \mathbf{b}_{0} \in \overline{\mathcal{A}}_{J}\right\},
$$

where $\overline{\mathcal{A}}_{J}$ is the alphabet associate to the heavy component $\bar{X}_{J}$ as defined above. The set $I_{J}^{(p)}$ can also be obtained as $I_{J} \cap \operatorname{Per}_{p}(X)$, where

$$
I_{J}:=\left\{\mathbf{b} \in X: \mathbf{b}_{0} \in \overline{\mathcal{A}}_{J}\right\},
$$

which is the set of all points whose orbit visits the heavy component $\bar{X}_{J}$ during an interval of time containing the origin.

We have the following important lemma. 
Lemma 1 (Factorization on Heavy Components). For $\beta$ large enough one has

$$
\mu_{\beta \phi+\psi}\left[\mathbf{b}_{0}^{n}\right]=\nu_{\psi, J}\left[\mathbf{b}_{0}^{n}\right] \mu_{\beta \phi+\psi}\left(I_{J}\right) \pm 2 e^{\beta \frac{\phi g}{4}},
$$

whenever $\left[\mathbf{b}_{0}^{n}\right] \cap \bar{X}_{J} \neq \emptyset$ for some $1 \leq J \leq N_{\phi}$, where $\phi_{g}<0$ is defined in (12).

Proof. Let $q=\left\lfloor e^{\eta \beta}\right\rfloor$ with $-\phi_{g} / 4<\eta<-\phi_{g} / 3$, and $p=k \bar{p} \geq n+e^{\beta \gamma}$ with $\gamma=s_{\phi}-\phi_{g} / 4$. In Appendix B.1 (Proposition 2) we prove that for $\beta$ large enough

$$
\sum_{\mathbf{a} \in \operatorname{Per}_{p}(X) \cap\left[\mathbf{b}_{0}^{n}\right]} e^{S_{p}(\beta \phi+\psi)(\mathbf{a})}=\exp \left( \pm 2 e^{\beta \frac{\phi_{g}}{4}}\right) \sum_{\mathbf{a} \in X^{(p, q)} \cap\left[\mathbf{b}_{0}^{n}\right]} e^{S_{p}(\beta \phi+\psi)(\mathbf{a})},
$$

for all $\mathbf{b} \in X$ such that $\left[\mathbf{b}_{0}^{n}\right] \cap \cup_{J=1}^{N} \bar{X}_{J} \neq \emptyset$. Using this and Proposition 1 we obtain

$$
\begin{aligned}
\mu_{\beta \phi+\psi}\left[\mathbf{b}_{0}^{n}\right] & =\frac{\sum_{\mathbf{a} \in X^{(p, q)} \cap\left[\mathbf{b}_{0}^{n}\right]} e^{S_{p}(\beta \phi+\psi)(\mathbf{a})}}{\sum_{\mathbf{a} \in \operatorname{Per}_{p}(X)} e^{S_{p}(\beta \phi+\psi)(\mathbf{a})}} \exp \left( \pm\left(2 e^{\beta \frac{\phi_{g}}{4}}+e^{-\beta\left(\gamma-s_{\phi}\right)}\right)\right) \\
& =\frac{\sum_{\mathbf{a} \in X^{(p, q)} \cap\left[\mathbf{b}_{0}^{n}\right]} e^{S_{p}(\beta \phi+\psi)(\mathbf{a})}}{\sum_{\mathbf{a} \in \operatorname{Per}_{p}(X)} e^{S_{p}(\beta \phi+\psi)(\mathbf{a})}} \exp \left( \pm 3 e^{\beta \frac{\phi_{g}}{4}}\right) .
\end{aligned}
$$

We organize the periodic orbits in $X^{(p, q)} \cap\left[\mathbf{b}_{0}^{n}\right]$ according to input-output vertices as follows. For each $\mathbf{a} \in X^{(p, q)}$ such that $\mathbf{a}_{0} \in \overline{\mathcal{A}}_{J}$, let

$$
i(\mathbf{a}):=\max \left\{0 \leq i<p:\left(\mathbf{a}_{i-1}, \mathbf{a}_{i}\right) \neq \overline{\mathcal{E}}_{J}\right\} \text { and } o(\mathbf{a}):=\min \left\{0 \leq i<p:\left(\mathbf{a}_{i}, \mathbf{a}_{i+1}\right) \neq \overline{\mathcal{E}}_{J}\right\} .
$$

Notice that $p-i(\mathbf{a})>q$ and $o(\mathbf{a}) \geq q-1$. Now, for $a, a^{\prime} \in \overline{\mathcal{A}}_{J}$ and $0 \leq j<i<p$, let

$$
\left[a, i ; a^{\prime}, j\right]_{J}^{(p)}:=\left\{\mathbf{a} \in X^{(p, q)}: \mathbf{a}_{0} \in \overline{\mathcal{A}}_{J}, i(\mathbf{a})=i, o(\mathbf{a})=j, \mathbf{a}_{i}=a \text { and } \mathbf{a}_{j}=a^{\prime}\right\}
$$

be the set of all $p$-periodic orbits in $\left[\mathbf{b}_{0}^{n}\right]$ intersecting the heavy component $\bar{X}_{J}$, leaving this component at vertex $a^{\prime}$ and time $j$, and entering the last time at vertex $a$ and time $i$.

It follows from (22) that for each $a, a^{\prime} \in \overline{\mathcal{A}}_{J}$ and $0 \leq j<i<p$ we have

$$
\begin{aligned}
& \sum_{\mathbf{a} \in\left[\mathbf{b}_{0}^{n}\right] \cap\left[a, i ; a^{\prime}, j\right]} e_{J}^{(p)} e^{S_{p}(\beta \phi+\psi)(\mathbf{a})} \\
= & \mathcal{M}_{\beta \phi+\psi, J}^{p-i}\left(a, \mathbf{b}_{0}\right)\left(\prod_{i=0}^{n-1} \mathcal{M}_{\beta \phi+\psi, J}\left(\mathbf{b}_{i}, \mathbf{b}_{i+1}\right)\right) \mathcal{M}_{\beta \phi+\psi, J}^{j-n}\left(\mathbf{b}_{n}, a^{\prime}\right) \mathcal{M}_{\beta \phi+\psi}^{i-j}\left(a^{\prime}, a\right) \\
= & \mathcal{M}_{\psi, J}^{p-i}\left(a, \mathbf{b}_{0}\right)\left(\prod_{i=0}^{n-1} \mathcal{M}_{\psi, J}\left(\mathbf{b}_{i}, \mathbf{b}_{i+1}\right)\right) \mathcal{M}_{\psi, J}^{j-n}\left(\mathbf{b}_{n}, a^{\prime}\right) e^{\overleftarrow{\phi}\left(a, a^{\prime}\right)} \mathcal{M}_{\beta \phi+\psi}^{i-j}\left(a^{\prime}, a\right),
\end{aligned}
$$

where $\overleftarrow{\phi}\left(a, a^{\prime}\right)$ is the compensation term, defined in (21), which relates $\mathcal{M}_{\psi, J}$ to $\mathcal{M}_{\beta \phi+\psi, J}$. Now since $\min (p-i, j-n, p-i+j) \geq q-n$, then using three times (23), and the definition (5) of $\nu_{J, \psi}$, 
we obtain

$$
\begin{aligned}
& \sum_{\mathbf{a} \in\left[\mathbf{b}_{0}^{n}\right] \cap\left[a, i ; a^{\prime}, j\right]_{J}^{(p)}} e^{S_{p}(\beta \phi+\psi)(\mathbf{a})} \\
& =\mathbf{v}_{\psi, J}(a) \mathbf{w}_{\psi, J}\left(\mathbf{b}_{0}\right)\left(\prod_{i=0}^{n-1} \mathcal{M}_{\psi, J}\left(\mathbf{b}_{i}, \mathbf{b}_{i+1}\right)\right) \mathbf{v}_{\psi, J}\left(\mathbf{b}_{n}\right) \mathbf{w}_{\psi, J}\left(a^{\prime}\right) e^{\overleftarrow{\phi}\left(a, a^{\prime}\right)} \\
& \times \mathcal{M}_{\beta \phi+\psi}^{i-j}\left(a^{\prime}, a\right) e^{ \pm 2 C_{\psi} \tau_{\psi}^{\left\lfloor(q-n) / p_{J}\right\rfloor}} \\
& =\nu_{\psi, J}\left[\mathbf{b}_{0}^{n}\right] \mathbf{v}_{J}(a) \mathbf{w}_{\psi, J}\left(a^{\prime}\right) \mathcal{M}_{\beta \phi+\psi}^{i-j}\left(a^{\prime}, a\right) e^{ \pm 2 C_{\psi} \tau_{\psi}^{\left\lfloor(q-n) / p_{J}\right\rfloor}} e^{\overleftarrow{\phi}\left(a, a^{\prime}\right)} \\
& =\nu_{\psi, J}\left[\mathbf{b}_{0}^{n}\right] \mathcal{M}_{\psi, J}\left(a, a^{\prime}\right)^{p-i+j} \mathcal{M}_{\beta \phi+\psi}^{i-j}\left(a^{\prime}, a\right) e^{\overleftarrow{\phi}\left(a, a^{\prime}\right)} e^{ \pm 3 C_{\psi} \tau_{\psi}^{\left\lfloor(q-n) / p_{J}\right\rfloor}} \\
& =\nu_{\psi, J}\left[\mathbf{b}_{0}^{n}\right] \mathcal{M}_{\beta \phi+\psi, J}\left(a, a^{\prime}\right)^{p-i+j} \mathcal{M}_{\beta \phi+\psi}^{i-j}\left(a^{\prime}, a\right) e^{ \pm 3 C_{\psi} \tau_{\psi}^{\left\lfloor(q-n) / p_{J}\right\rfloor}} \\
& =e^{ \pm 3 C_{\psi} \tau_{\psi}^{\left\lfloor(q-n) / p_{J}\right\rfloor}} \nu_{\psi, J}\left[\mathbf{b}_{0}^{n}\right] \sum_{\mathbf{a} \in\left[a, i ; a^{\prime}, j\right]_{J}^{(p)}} e^{S_{p}(\beta \phi+\psi)(\mathbf{a})} .
\end{aligned}
$$

With this, (25), and taking into account that

$$
\left\{\mathbf{a} \in X^{(p, q)}: \mathbf{a}_{0} \in \overline{\mathcal{A}}_{J}\right\}=\bigcup_{a, a^{\prime} \in \overline{\mathcal{A}}_{J}} \bigcup_{q \leq j \leq i \leq p-q}\left[a, i ; a^{\prime}, j\right]_{J}^{(p)},
$$

it follows that

$$
\begin{aligned}
\mu_{\beta \phi+\psi}\left[\mathbf{b}_{0}^{n}\right]=\nu_{\psi, J}\left[\mathbf{b}_{0}^{n}\right] \mathcal{P}_{\beta \phi+\psi}^{(p)} & \left(\left\{\mathbf{a} \in X^{(p, q)}: \mathbf{a}_{0} \in \overline{\mathcal{A}}_{J}\right\}\right) \\
& \times \exp \left( \pm 3\left(e^{\beta \frac{\phi_{g}}{4}}+C_{\psi} \tau_{\psi}^{\left\lfloor(q-n) / p_{J}\right\rfloor}\right)\right) .
\end{aligned}
$$

Now, (24) implies that

$$
\mathcal{P}_{\beta \phi+\psi}^{(p)}\left(\left\{\mathbf{a} \in X^{(p, q)}: \mathbf{a}_{0} \in \overline{\mathcal{A}}_{J}\right\}\right)=\mathcal{P}_{\beta \phi+\psi}^{(p)}\left(I_{J}^{(p)}\right) \exp \left( \pm 2 e^{-\beta \frac{\phi_{g}}{2}}\right),
$$

therefore

$$
\mu_{\beta \phi+\psi}\left[\mathbf{b}_{0}^{n}\right]=\nu_{\psi, J}\left[\mathbf{b}_{0}^{n}\right] \mathcal{P}_{\beta \phi+\psi}^{(p)}\left(I_{J}^{(p)}\right) \exp \left( \pm\left(5 e^{\beta \frac{\phi_{g}}{4}}+3 C_{\psi} \tau_{\psi}^{\left\lfloor(q-n) / p_{J}\right\rfloor}\right)\right),
$$

for each $q=\left\lfloor e^{\eta \beta}\right\rfloor$, with $-\phi_{g} / 4<\eta<-\phi_{g} / 3$.

Since $\tau_{\phi}<1$, then $C_{\psi} \tau_{\psi}^{\left\lfloor(q-n) / p_{J}\right\rfloor} \leq e^{\beta \phi_{g} / 4}$ if we take $q \geq \beta^{2}$ and $\beta$ large enough. Therefore

$$
\mu_{\beta \phi+\psi}\left[\mathbf{b}_{0}^{n}\right]=\nu_{\psi, J}\left[\mathbf{b}_{0}^{n}\right] \mathcal{P}_{\beta \phi+\psi}^{(p)}\left(I_{J}^{(p)}\right) \exp \left( \pm 6 e^{\beta \frac{\phi_{g}}{4}}\right),
$$

and since $\mathcal{P}_{\beta \phi+\psi}^{(p)}\left(I_{J}^{(p)}\right)=\mathcal{P}_{\beta \phi+\psi}^{(p)}\left(I_{J}\right)$, then we obtain

$$
\mu_{\beta \phi+\psi}\left[\mathbf{b}_{0}^{n}\right]=\nu_{\psi, J}\left[\mathbf{b}_{0}^{n}\right] \mathcal{P}_{\beta \phi+\psi}^{(p)}\left(I_{J}\right) \exp \left( \pm 6 e^{\beta \frac{\phi_{g}}{4}}\right)
$$


for each $\mathbf{b} \in X$ such that $\left[\mathbf{b}_{0}\right] \cap \bar{X}_{J} \neq \emptyset$.

Notice now that $X \backslash I_{J}:=\left\{\mathbf{b} \in X: \mathbf{b}_{0} \notin \overline{\mathcal{A}}_{J}\right\}$ is a union of cylinder sets, therefore Proposition 1 implies that for $\beta$ large enough

$$
1-\mathcal{P}_{\beta \phi+\psi}^{(p)}\left(I_{J}\right)=\left(1-\mu_{\beta \phi+\psi}\left(I_{J}\right)\right) \exp \left( \pm e^{-\beta\left(\gamma-s_{\phi}\right)}\right) .
$$

Hence,

$$
\mathcal{P}_{\beta \phi+\psi}^{(p)}\left(I_{J}\right)=\mu_{\beta \phi+\psi}\left(I_{J}\right)+\left(1-\mu_{\beta \phi+\psi}\left(I_{J}\right)\right)\left(1-\exp \left( \pm e^{-\beta\left(\gamma-s_{\phi}\right)}\right)\right) .
$$

Since

$$
\exp \left( \pm e^{-\beta\left(\gamma-s_{\phi}\right)}\right)=1 \pm \frac{3}{2} e^{-\beta\left(\gamma-s_{\phi}\right)} \text { and } \exp \left( \pm 6 e^{\beta \frac{\phi_{g}}{4}}\right)=1 \pm 9 e^{\beta \frac{\phi_{g}}{4}}
$$

for $\beta$ large enough, then, substituting in (26) and (27) we obtain

$$
\mu_{\beta \phi+\psi}\left[\mathbf{b}_{0}^{n}\right]=\nu_{\psi, J}\left[\mathbf{b}_{0}^{n}\right] \mu_{\beta \phi+\psi}\left(I_{J}\right) \pm \frac{3}{2} e^{\beta \frac{\phi_{g}}{4}}\left(1+9 e^{\beta \frac{\phi_{g}}{4}}\right)
$$

from which the result follows by taking $\beta$ big enough.

\subsection{Excursion Potentials.}

We will now replace $M_{\beta \phi+\psi}$ by another transition matrix closed to it, whose entries can be explicitly computed in terms of the renormalized protentials defined above. Let us recall their definitions.

Let $\mathbf{w}_{\beta \phi+\psi}$ and $\mathbf{v}_{\beta \phi+\psi}$ be the left and right maximal eigenvectors of our original transition matrix $\mathcal{M}_{\beta \phi+\psi}$ on the alphabet $\mathcal{A}$. Let $\tilde{\mathcal{A}}:=\mathcal{A} \backslash \bigsqcup_{J=1}^{N_{\phi}} \overline{\mathcal{A}}_{J}$, and $\tilde{\mathcal{M}}_{\beta \phi+\psi}<\mathcal{M}_{\beta \phi+\psi}$ the submatrix of $\mathcal{M}_{\beta \phi+\psi}$ obtained by excluding all the heavy components.

Now, for each heavy component $1 \leq J \leq N_{\phi}$ and $a, a^{\prime} \in \overline{\mathcal{A}}_{J}$ let

$$
\tilde{\mathbf{w}}_{\beta \phi+\psi}(a):=\sum_{b \in \mathcal{A}} \mathbf{w}_{\beta \phi+\psi}(b) \tilde{\mathcal{M}}_{\beta \phi+\psi}(b, a) \text {, and } \tilde{\mathbf{v}}_{\beta \phi+\psi}(a):=\sum_{b \in \mathcal{A}} \tilde{\mathcal{M}}_{\beta \phi+\psi}\left(a^{\prime}, b\right) \mathbf{v}_{\beta \phi+\psi}(b) .
$$

Let us recall the definition of the transition term (see (9) above). For $a^{\prime} \in \overline{\mathcal{A}}_{J}$ and $c \in \overline{\mathcal{A}}_{K}$, let $\operatorname{Path}\left[a^{\prime}, c\right]$ be the set of all simple paths, starting at $a^{\prime}$ and ending at $c$, with no arrows in $\overline{\mathcal{E}}_{\phi}$ other than $a^{\prime}$ and $c$. The $\left(a^{\prime}, c\right)$-transition term is the maximum

$$
\vec{\phi}\left(a^{\prime}, c\right):=\max \left\{\phi\left(a^{\prime} \rightarrow c\right): a^{\prime} \rightarrow c \in \operatorname{Path}\left[a^{\prime}, c\right]\right\},
$$

where $\phi\left(a^{\prime} \rightarrow c\right):=\phi\left(a^{\prime}, b_{1}\right)+\sum_{i=1}^{m-1} \phi\left(b_{i}, b_{i+1}\right)+\phi\left(b_{m}, c\right)$ for $a \rightarrow c=\left(a^{\prime}, b_{1}, \ldots, b_{m}, c\right)$.

Let $\mathcal{M}_{\psi}: \mathcal{A} \times \mathcal{A} \rightarrow \mathbb{R}^{+}$be defined in the same way as $\mathcal{M}_{\beta \phi+\psi}$, with $\psi$ replacing $\beta \phi+\psi$, and let $\overline{\mathcal{M}}_{\psi}$ be the restriction of $\mathcal{M}_{\psi}$ to all the transitive components of $\bar{X}$, either heavy or not. Because of the normalization $P(\psi \mid \bar{X})=0$, we have $\sum_{n=1}^{\infty} \overline{\mathcal{M}}_{\psi}^{n}(b, b)<\infty$, for each $b \notin \tilde{\mathcal{A}}$. This is due to the fact that non-heavy transitive components of $\bar{X}$ have maximal eigenvalue strictly smaller than 1 .

Let us now fix, for each heavy component $1 \leq J \leq N_{\phi}$, a central vertex $c_{J} \in \overline{\mathcal{A}}_{J}$. Using this vertex, define, for each $a^{\prime} \in \overline{\mathcal{A}}_{J}$, the central term, whose definition we remind here:

$$
\overleftarrow{\phi}\left(a^{\prime}\right):=\phi\left(c_{J} \rightsquigarrow a^{\prime}\right) \text { with } c_{J} \rightsquigarrow a^{\prime} \text { a path in } \mathcal{G}_{J} \text { from } c_{J} \text { to } a
$$


It is easy to see that the central term satisfies the relation

$$
\overleftarrow{\phi}\left(a^{\prime}\right)=\overleftarrow{\phi}(a)+\overleftarrow{\phi}\left(a, a^{\prime}\right)
$$

We are now ready to define the excursion potentials $\tilde{\phi}, \tilde{\psi}: \mathcal{A}_{\text {ext }} \times \mathcal{A}_{\text {ext }} \rightarrow \mathbb{R}$ such that

$$
\begin{aligned}
\tilde{\phi}\left(\left[a, a^{\prime}\right]_{J},\left[c, c^{\prime}\right]_{K}\right) & :=\overleftarrow{\phi}\left(a^{\prime}\right)+\vec{\phi}\left(a^{\prime}, c\right)-\overleftarrow{\phi}(c) \\
\left.\tilde{\psi}\left(\left[a, a^{\prime}\right]_{J},\left[c, c^{\prime}\right]_{K}\right)\right) & :=\log \left(\sum_{a^{\prime} \rightarrow c \in \overline{\operatorname{Path}}\left[a^{\prime}, c\right]} e^{\psi\left(a^{\prime} \rightarrow c\right)+P_{\psi}\left(a^{\prime} \rightarrow c\right)}\right)+\log \left(\mathbf{v}_{\psi, J}\left(a^{\prime}\right) \mathbf{w}_{\psi, K}(c)\right)
\end{aligned}
$$

where $\left.P_{\psi}\left(a^{\prime} \rightarrow c\right)\right)$ is the transition pressure defined in $(7)$ and where $\overline{\operatorname{Path}}\left[a^{\prime}, c\right] \subset \operatorname{Path}\left[a^{\prime}, c\right]$ is the set of all simple paths from $a^{\prime}$ to $c$ maximizing $\phi$. As usual, we will denote by $M_{\beta \tilde{\phi}+\tilde{\psi}}$ the transition matrix defined by the potential $\beta \tilde{\phi}+\tilde{\psi}$.

Recall the notation $\phi(C):=\sum_{i=0}^{|C|-1} \phi\left(b_{i}, b_{i+1}\right)$ for the circuit $C=\left(b_{0}, \ldots, b_{|C|-1}\right)$.

Lemma 2 (Approximated Cohomology). For $\beta$ large enough, and for all a, a $\in \overline{\mathcal{A}}_{J}, c, c^{\prime} \in \overline{\mathcal{A}}_{K}$, $1 \leq J, K \leq N_{\phi}$, we have

$$
M_{\beta \phi+\psi}\left(\left[a, a^{\prime}\right]_{J},\left[c, c^{\prime}\right]_{K}\right)=M_{\beta \tilde{\phi}+\tilde{\psi}}\left(\left[a, a^{\prime}\right]_{J},\left[c, c^{\prime}\right]_{K}\right) \frac{e^{\beta \overleftarrow{\phi}\left(c^{\prime}\right)} \mathbf{v}_{\psi, K}\left(c^{\prime}\right) \tilde{\mathbf{v}}_{\beta \phi+\psi}\left(c^{\prime}\right)}{e^{\beta \overleftarrow{\phi}\left(a^{\prime}\right)} \mathbf{v}_{\psi, J}\left(a^{\prime}\right) \tilde{\mathbf{v}}_{\beta \phi+\psi}\left(a^{\prime}\right)} \frac{e^{ \pm e^{-\beta \delta}}}{\rho_{\beta \phi+\psi}-1}
$$

where

$$
\delta:=\frac{1}{6} \min \left\{\left|\phi(C)-\phi\left(C^{\prime}\right)\right|: C, C^{\prime} \in \mathcal{C}, \phi(C) \neq \phi\left(C^{\prime}\right), \text { and }|C|,\left|C^{\prime}\right| \leq 2 \# \mathcal{A}\right\} .
$$

Proof. First of all notice that

$$
\begin{aligned}
& \mu_{\beta \phi+\psi}\left(\left[\left[a, a^{\prime}\right]_{J},\left[c, c^{\prime}\right]_{K}\right]\right)= \tilde{\mathbf{w}}_{\beta \phi+\psi}(a)\left(\sum_{k=0}^{\infty} \frac{\mathcal{M}_{\beta \phi+\psi, J}^{k}\left(a, a^{\prime}\right)}{\rho_{\beta \phi+\psi}^{k}}\right)\left(\sum_{k=0}^{\infty} \frac{\tilde{\mathcal{M}}_{\beta \phi+\psi}^{k}\left(a^{\prime}, c\right)}{\rho_{\beta \phi+\psi}^{k}}\right) \\
& \quad \times\left(\sum_{k=0}^{\infty} \frac{\mathcal{M}_{\beta \phi+\psi, K}^{k}\left(c, c^{\prime}\right)}{\rho_{\beta \phi+\psi}^{k}}\right) \tilde{\mathbf{v}}_{\beta \phi+\psi}\left(c^{\prime}\right), \\
& \mu_{\beta \phi+\psi}\left(\left[a, a^{\prime}\right]_{J}\right)=\tilde{\mathbf{w}}_{\beta \phi+\psi}(a)\left(\sum_{k=0}^{\infty} \frac{\mathcal{M}_{\beta \phi+\psi, J}^{k}\left(a, a^{\prime}\right)}{\rho_{\beta \phi+\psi}^{k}}\right) \tilde{\mathbf{v}}_{\beta \phi+\psi}\left(a^{\prime}\right) .
\end{aligned}
$$

Hence, taking into account (22) and (28) we have

$$
\begin{aligned}
& M_{\beta \phi+\psi}\left(\left[\left[a, a^{\prime}\right]_{J},\left[c, c^{\prime}\right]_{K}\right]\right)= \\
& \quad\left(\sum_{k=0}^{\infty} \frac{\tilde{\mathcal{M}}_{\beta \phi+\psi}^{k}\left(a^{\prime}, c\right)}{\rho_{\beta \phi+\psi}^{k}}\right)\left(\sum_{k=1}^{\infty} \frac{\mathcal{M}_{\psi, K}^{k}\left(c, c^{\prime}\right)}{\rho_{\beta \phi+\psi}^{k}}\right) \frac{\tilde{\mathbf{v}}_{\beta \phi+\psi}\left(c^{\prime}\right) e^{\beta\left(\overleftarrow{\phi}\left(c^{\prime}\right)-\overleftarrow{\phi}(c)\right)}}{\tilde{\mathbf{v}}_{\beta \phi+\psi}\left(a^{\prime}\right)} .
\end{aligned}
$$

We now deal with

$$
Q\left(a^{\prime}, c\right):=\sum_{k=0}^{\infty} \frac{\tilde{\mathcal{M}}_{\beta \phi+\psi}^{k}\left(a^{\prime}, c\right)}{\rho_{\beta \phi+\psi}^{k}}, \quad R\left(c, c^{\prime}\right):=\sum_{k=1}^{\infty} \frac{\mathcal{M}_{\psi, K}^{k}\left(c, c^{\prime}\right)}{\rho_{\beta \phi+\psi}^{k}} .
$$


Notice that $Q\left(a^{\prime}, c\right)=\sum_{a^{\prime} \rightsquigarrow c \in \widehat{\operatorname{Path}}\left[a^{\prime}, c\right]} e^{\beta \phi\left(a^{\prime} \rightsquigarrow c\right)+\psi\left(a^{\prime} \rightsquigarrow c\right)-\left|a^{\prime} \rightsquigarrow c\right| \log \left(\rho_{\beta \phi+\psi}\right)}$, where $\widehat{\operatorname{Path}}\left[a^{\prime}, c\right]$ denotes the collection of all paths in $\mathcal{G}_{X}$ going from $a^{\prime}$ to $c$, with no arrows in $\overline{\mathcal{E}}_{\phi}$ (defined in (1)).

Any path $a^{\prime} \rightsquigarrow c \in \widehat{\operatorname{Path}}\left[a^{\prime}, c\right]$ can be decomposed into an elementary path $a^{\prime} \rightarrow c=\left(a, b_{1}, \ldots, b_{m-1}, c\right) \in$ $\operatorname{Path}\left[a^{\prime}, c\right]$ and a sum of circuits $b_{i_{1}} \circlearrowleft+b_{i_{2}} \circlearrowleft+\cdots+b_{i_{\ell}} \circlearrowleft$, with no arrows in $\overline{\mathcal{E}}_{\phi}$. Let us denote by $\tilde{\mathcal{C}}_{b}$ the collection of all the circuits in $\mathcal{G}_{X}$ with base point $b$, and with no arrows in $\overline{\mathcal{E}}_{\phi}$. Taking this into account, we can rewrite $Q\left(a^{\prime}, c\right)$ as

$$
Q\left(a^{\prime}, c\right)=\sum_{a^{\prime} \rightarrow c \in \operatorname{Path}\left[a^{\prime}, c\right]} e^{\beta \phi\left(a^{\prime} \rightarrow c\right)+\psi\left(a^{\prime} \rightarrow c\right)-\left|a^{\prime} \rightarrow c\right| \log \left(\rho_{\beta \phi+\psi}\right)} \sum_{b \in a^{\prime} \rightarrow c} \sum_{k=0}^{\infty} \frac{\tilde{\mathcal{M}}_{\beta \phi+\psi}^{k}(b, b)}{\rho_{\beta \phi+\psi}^{k}},
$$

where Path $\left[a^{\prime}, c\right]$ denotes the collection of all simple paths in $\mathcal{G}_{X}$ going from $a^{\prime}$ to $c$, and $b \in a^{\prime} \rightarrow c$ means that the path $a^{\prime} \rightarrow c$ passes through the vertex $b$. By Proposition 4 (Appendix B.3) we have

$$
\sum_{k=0}^{\infty} \frac{\tilde{\mathcal{M}}_{\beta \phi+\psi}^{k}(b, b)}{\rho_{\beta \phi+\psi}^{k}}=\sum_{k=0}^{\infty} \overline{\mathcal{M}}_{\psi}^{k}(b, b) \pm D e^{\beta \phi_{g}},
$$

for $\beta$ large enough, for all $b \notin \bigsqcup_{J=1}^{N_{\phi}} \overline{\mathcal{A}}_{J}:=\bigsqcup_{J=1}^{N_{\phi}} \overline{\mathcal{A}}_{J}$, and for some constant $D>0$. Therefore,

$$
\begin{aligned}
\sum_{b \in a^{\prime} \rightarrow c} \sum_{k=0}^{\infty} \frac{\tilde{\mathcal{M}}_{\beta \phi+\psi}^{k}(b, b)}{\rho_{\beta \phi+\psi}^{k}} & =e^{P_{\psi}\left(a^{\prime} \rightarrow b\right)}\left(1 \pm \frac{\left|a^{\prime} \rightarrow c\right| D}{e^{P_{\psi}\left(a^{\prime} \rightarrow c\right)}} e^{\beta \phi_{g}}\right) \\
& =e^{P_{\psi}\left(a^{\prime} \rightarrow c\right)} \exp \left( \pm e^{\beta \frac{3 \phi_{g}}{4}}\right)
\end{aligned}
$$

for all $\beta$ large enough.

Now, if $a^{\prime} \rightarrow c \in \operatorname{Path}\left[a^{\prime}, c\right]$ is not maximal, then we necessarily have

$$
\phi\left(a^{\prime} \rightarrow c\right) \leq \vec{\phi}\left(a^{\prime}, c\right)-\min \left\{\left|\phi(C)-\phi\left(C^{\prime}\right)\right|: \phi(C) \neq \phi\left(C^{\prime}\right), C, C^{\prime} \in \mathcal{C}_{0}\left[a^{\prime}, c\right]\right\},
$$

where $\mathcal{C}_{0}\left[a^{\prime}, c\right]$ denote the set of circuits formed by a simple path from $a^{\prime}$ to $c$ and followed by a simple path from $c$ to $a^{\prime}$. Since $6 \delta<\min \left\{\left|\phi(C)-\phi\left(C^{\prime}\right)\right|: \phi(C) \neq \phi\left(C^{\prime}\right), C, C^{\prime} \in \mathcal{C}_{0}\left[a^{\prime}, c\right]\right\}$, then

$$
\begin{aligned}
\sum_{a^{\prime} \rightarrow c \in \operatorname{Path}\left[a^{\prime}, c\right]} e^{\beta \phi\left(a^{\prime} \rightarrow c\right)+\psi\left(a^{\prime} \rightarrow c\right)+P_{\psi}\left(a^{\prime} \rightarrow c\right)}= & e^{\beta \vec{\phi}\left(a^{\prime}, c\right)} \sum_{a^{\prime} \rightarrow c \in \overline{\operatorname{Path}}\left[a^{\prime}, c\right]} e^{\psi\left(a^{\prime} \rightarrow c\right)+P_{\psi}\left(a^{\prime} \rightarrow c\right)} \times \\
& \left(1 \pm e^{\beta 6 \delta} \frac{\sum_{a^{\prime} \rightarrow c \in \operatorname{Path}\left[a^{\prime}, c\right]} e^{\psi\left(a^{\prime} \rightarrow c\right)+P_{\psi}\left(a^{\prime} \rightarrow c\right)}}{\sum_{a^{\prime} \rightarrow c \in \overline{\operatorname{Path}}\left[a^{\prime}, c\right]} e^{\psi\left(a^{\prime} \rightarrow c\right)+P_{\psi}\left(a^{\prime} \rightarrow c\right)}}\right) \\
= & \exp \left( \pm e^{-\beta 5 \delta}\right) e^{\beta \vec{\phi}\left(a^{\prime}, c\right)} \sum_{a^{\prime} \rightarrow c \in \overline{\operatorname{Path}}\left[a^{\prime}, c\right]} e^{\psi\left(a^{\prime} \rightarrow c\right)+P_{\psi}\left(a^{\prime} \rightarrow c\right)}
\end{aligned}
$$

for $\beta$ large enough. Proposition 3 (Appendix B.2) implies that $\rho_{\beta \phi+\psi}=1 \pm e^{\beta \phi_{g} / 2}$, for $\beta$ large enough. On the other hand, since $a^{\prime} \rightarrow c \in \operatorname{Path}\left[a^{\prime}, c\right]$ is a simple path, then $\left|a^{\prime} \rightarrow c\right| \leq \# \mathcal{A}$, therefore, for $\beta$ large enough, $\left|a^{\prime} \rightarrow c\right| \log \left(\rho_{\beta \phi+\psi}\right)= \pm e^{\beta \frac{2 \phi_{g}}{5}}$. Taking this into account, and using 
(29), we obtain

$$
\begin{aligned}
Q\left(a^{\prime}, c\right) & =\exp \left( \pm\left(e^{-\beta 5 \delta}+e^{\beta 2 \phi_{g} / 5}\right)\right) e^{\beta \vec{\phi}\left(a^{\prime}, c\right)} \sum_{a^{\prime} \rightarrow c \in \overline{\operatorname{Path}}\left[a^{\prime}, c\right]} e^{\psi\left(a^{\prime} \rightarrow c\right)+P_{\psi}\left(a^{\prime} \rightarrow c\right)} \\
& =\exp \left( \pm e^{-\beta 2 \delta}\right) e^{\beta \vec{\phi}\left(a^{\prime}, c\right)} \sum_{a^{\prime} \rightarrow c \in \overline{\operatorname{Path}}\left[a^{\prime}, c\right]} e^{\psi\left(a^{\prime} \rightarrow c\right)+P_{\psi}\left(a^{\prime} \rightarrow c\right)}
\end{aligned}
$$

for $\beta$ large enough. we used the fact that $-2 \phi_{g} / 5 \geq 12 \delta / 5>2 \delta$.

The factor $R\left(c, c^{\prime}\right):=\sum_{k=1}^{\infty} \mathcal{M}_{\psi, K}^{k}\left(c, c^{\prime}\right) / \rho_{\beta \phi+\psi}^{k}$ can be treated as follows. Fix $k_{0}=k_{0}(\beta)$ so that $C_{\psi} \tau_{\psi}^{k_{0}} \leq e^{\beta \phi_{g}}$, with $C_{\psi}$ and $\tau_{\psi}$ as in (23). Since $\tau_{\psi}<1$, then we can choose $k_{0}$ proportional to $\beta$. Thus, from the cited lemma we obtain

$$
\sum_{k=k_{0}}^{\infty} \frac{\mathcal{M}_{\psi, K}^{k}\left(c, c^{\prime}\right)}{\rho_{\beta \phi+\psi}^{k}}=\exp \left( \pm e^{\beta \phi_{g}}\right) \mathbf{w}_{\psi, K}(c) \mathbf{v}_{\psi, K}\left(c^{\prime}\right) \sum_{k=k_{0}}^{\infty} \rho_{\beta \phi+\psi}^{-\left(p_{K} k+r\right)},
$$

where $r=r\left(c, c^{\prime}\right)$ is the smallest integer such that $\mathcal{M}_{\psi, K}^{r}\left(c, c^{\prime}\right)>0$. Now, for $k<k_{0}$ we have

$$
\sum_{k=0}^{k_{0}-1} \frac{\mathcal{M}_{\psi, K}^{k}\left(c, c^{\prime}\right)}{\rho_{\beta \phi+\psi}^{k}}<\sum_{k=0}^{k_{0}-1} \mathcal{M}_{\psi, K}^{p_{K} k+r}\left(c, c^{\prime}\right) \leq \frac{k_{0} \max _{k \in \mathbb{N}} \mathcal{M}_{\psi, K}^{k}\left(c, c^{\prime}\right)}{p_{K}},
$$

where $\max _{k \in \mathbb{N}} \mathcal{M}_{\psi, K}^{k}\left(c, c^{\prime}\right)<\infty$ follows from the fact that $\max \left|\operatorname{spec}\left(\mathcal{M}_{\psi, K}\right)\right|=1$.

By Proposition 3 one has $\rho_{\beta \phi+\psi} \leq 1+e^{\beta \frac{\phi_{g}}{2}}$. From this, after a few computations, it follows that $\sum_{k=0}^{\infty} \rho_{\beta \phi+\psi}^{-\left(p_{K} k+r\right)} \geq 2^{-r} e^{-\beta \frac{\phi g}{2}} / p_{K}$. Then, since $k_{0}$ is proportional to $\beta$, by taking $\beta$ large enough we obtain

and from this

$$
\sum_{k=0}^{k_{0}-1} \frac{\mathcal{M}_{K}^{k}\left(c, c^{\prime}\right)}{\rho_{\beta \phi+\psi}^{k}}<e^{\beta \frac{\phi_{g}}{3}} \mathbf{w}_{\psi, K}(c) \mathbf{v}_{\psi, K}\left(c^{\prime}\right) \sum_{k=0}^{\infty} \rho_{\beta \phi+\psi}^{-\left(p_{K} k+r\right)},
$$

$$
\begin{aligned}
& \left(e^{e^{\beta \phi_{g}}}-e^{\beta \frac{\phi_{g}}{3}}\right) \mathbf{w}_{\psi, K}(c) \mathbf{v}_{\psi, K}\left(c^{\prime}\right) \frac{\rho_{\beta \phi+\psi}^{p_{K}-r}}{\rho_{\beta \phi+\psi}-1} \leq \sum_{k=0}^{\infty} \frac{\mathcal{M}_{K}^{k}\left(c, c^{\prime}\right)}{\rho_{\beta \phi+\psi}^{k}}, \\
& \left(e^{e^{\beta \phi_{g}}}+e^{\beta \frac{\phi_{g}}{3}}\right) \mathbf{w}_{\psi, K}(c) \mathbf{v}_{\psi, K}\left(c^{\prime}\right) \frac{\rho_{\beta \phi+\psi}^{p_{K}-r}}{\rho_{\beta \phi+\psi}-1} \geq \sum_{k=0}^{\infty} \frac{\mathcal{M}_{K}^{k}\left(c, c^{\prime}\right)}{\rho_{\beta \phi+\psi}^{k}} .
\end{aligned}
$$

From these two inequalities, and taking into account that $\rho_{\beta \phi+\psi}=\exp \left( \pm e^{\beta \phi_{g} / 2}\right)$, it follows that

$$
\begin{aligned}
R\left(c, c^{\prime}\right) & =\exp \left( \pm 3 e^{-\beta \frac{4 \delta}{3}}\right) \mathbf{w}_{\psi, K}(c) \mathbf{v}_{\psi, K}\left(c^{\prime}\right) \frac{\rho_{\beta \phi+\psi}^{p_{K}-r}}{\rho_{\beta \phi+\psi}-1} \\
& =\frac{\exp \left( \pm\left(3 e^{-\beta \frac{4 \delta}{3}}+\left(p_{K}-r\right) e^{\beta \phi_{g} / 2}\right)\right)}{\rho_{\beta \phi+\psi}-1} \mathbf{w}_{\psi, K}(c) \mathbf{v}_{\psi, K}\left(c^{\prime}\right) \\
& =\frac{\exp \left( \pm 4 e^{-\beta \frac{4 \delta}{3}}\right)}{\rho_{\beta \phi+\psi}-1} \mathbf{w}_{\psi, K}(c) \mathbf{v}_{\psi, K}\left(c^{\prime}\right)
\end{aligned}
$$


for $\beta$ large enough. The result follows from the bounds (30) and (31).

\subsection{Projecting the Excursion System.}

The aim here is to "compress" the excursion system defined on $\mathcal{A}_{\text {ext }}(14)$.

Recall that $\mathcal{A}^{\prime}=\left\{1,2, \ldots, N_{\phi}\right\}$ is the index set of the heavy components and that the projection $\pi: \mathcal{A}_{\text {ext }} \rightarrow \mathcal{A}^{\prime}$ is such that $\pi\left(\left[a, a^{\prime}\right]_{J}\right)=J$ for all $1 \leq J \leq N_{\phi}$ and $a, a^{\prime} \in \overline{\mathcal{A}}_{J}$, and extend it coordinatewise to $\left(\mathcal{A}_{\text {ext }}\right)^{\mathbb{Z}}$. Let

$$
\bar{\mu}_{\beta \tilde{\phi}+\tilde{\psi}}:=\mu_{\beta \tilde{\phi}+\tilde{\psi}} \circ \pi^{-1}
$$

denote the pull back of the measure $\mu_{\beta \tilde{\phi}+\tilde{\psi}}$ under the projection $\pi$. Since

$$
\bar{\mu}_{\beta \tilde{\phi}+\tilde{\psi}}[J]=\sum_{a, a^{\prime} \in \overline{\mathcal{A}}_{J}} \mu_{\beta \tilde{\phi}+\tilde{\psi}}\left(\left[\left[a, a^{\prime}\right]_{J}\right]\right) \equiv \sum_{\mathbf{d}, \mathbf{e} \in \overline{\mathcal{A}}_{J}} \mu_{\beta \tilde{\phi}+\tilde{\psi}}^{(1)}\left(\left[a, a^{\prime}\right]_{J}\right),
$$

for each $J \in \mathcal{A}^{\prime}$ and $a, a^{\prime} \in \overline{\mathcal{A}}_{J}$, then (19) (fourth step in the proof of the Renormalization Lemma) can be written as

$$
\mu_{\beta \phi+\psi}\left[\mathbf{b}_{0}^{n}\right]=\nu_{\psi, J}\left[\mathbf{b}_{0}^{n}\right] \bar{\mu}_{\beta \tilde{\phi}+\tilde{\psi}}[J] \pm 9\left(\# \mathcal{A}_{\mathrm{ext}}-1\right)
$$

for $\beta$ large enough and whenever $\left[\mathbf{b}_{0}^{n}\right] \cap \bar{X}_{J} \neq \emptyset$ for some $1 \leq J \leq N_{\phi}$.

We have the following.

Lemma 3 (The Projection is Markovian). The pull back $\bar{\mu}_{\beta \tilde{\phi}+\tilde{\psi}}$ of the measure $\mu_{\beta \tilde{\phi}+\tilde{\psi}}$ under the projection $\pi:\left(\mathcal{A}_{\text {ext }}\right)^{\mathbb{Z}} \rightarrow\left(\mathcal{A}^{\prime}\right)^{\mathbb{Z}}$, coincides with the Gibbs state defined by the 2-symbol potential $\beta \tilde{\phi}+\tilde{\psi}: \mathcal{A}_{\text {ext }} \times \mathcal{A}_{\text {ext }} \rightarrow \mathbb{R}$.

Proof. The Gibbs state (Markov measure) associated to $\beta \tilde{\phi}+\tilde{\psi}$, where $\tilde{\phi}$ and $\tilde{\psi}$ are the approximate excursion potentials, is defined by

$$
\begin{aligned}
\mu_{\beta \tilde{\phi}+\tilde{\psi}}\left[\left[a_{0}, a_{0}^{\prime}\right]_{J_{0}}\right. & \left.\cdots\left[a_{n}, a_{n}^{\prime}\right]_{J_{n}}\right]:= \\
& \mathbf{w}_{\beta \tilde{\phi}+\tilde{\psi}}\left(\left[a_{0}, a_{0}^{\prime}\right]_{J_{0}}\right) \frac{\prod_{i=0}^{n-1} M_{\beta \tilde{\phi}+\tilde{\psi}}\left(\left[a_{i}, a_{i}^{\prime}\right]_{J_{i}},\left[a_{i+1}, a_{i+1}^{\prime}\right]_{J_{i+1}}\right)}{\rho_{\beta \tilde{\phi}+\tilde{\psi}}^{n}} \mathbf{v}_{\beta \tilde{\phi}+\tilde{\psi}}\left(\left[a_{n}, a_{n}^{\prime}\right]_{J_{n}}\right),
\end{aligned}
$$

with $\rho_{\beta \tilde{\phi}+\tilde{\psi}}$ the maximal eigenvalue of associated transition matrix $M_{\beta \tilde{\phi}+\tilde{\psi}}$.

Since the matrix element $M_{\beta \tilde{\phi}+\tilde{\psi}}\left(\left[a, a^{\prime}\right]_{J},\left[c, c^{\prime}\right]_{K}\right)$ depends only on the internal symbols $a^{\prime}$ and $c$, we can write $M_{\beta \tilde{\phi}+\tilde{\psi}}\left(a_{J}^{\prime}, c_{K}\right)$ instead of $M_{\beta \tilde{\phi}+\tilde{\psi}}\left(\left[a, a^{\prime}\right]_{J},\left[c, c^{\prime}\right]_{K}\right)$. Now, for each $1 \leq J \leq N_{\phi}$ and $a^{\prime} \in \overline{\mathcal{A}}_{J}$ fixed, let $\hat{\mathbf{w}}_{\beta \tilde{\phi}+\psi}\left(a_{J}\right):=\sum_{a \in \overline{\mathcal{A}}_{J}} \mathbf{w}_{\beta \tilde{\phi}+\tilde{\psi}}\left(\left[a, a^{\prime}\right]_{J}\right)$. Then, since $\mathbf{w}_{\beta \tilde{\phi}+\tilde{\psi}}$ is the left invariant 
vector associated to the maximal eigenvalue of $M_{\beta \tilde{\phi}+\tilde{\psi}}$, we have

$$
\begin{aligned}
\mathbf{w}_{\beta \tilde{\phi}+\tilde{\psi}}\left(\left[a, a^{\prime}\right]_{K}\right) & =\frac{1}{\rho_{\beta \tilde{\phi}+\tilde{\psi}}} \sum_{J ; a, a^{\prime} \in \overline{\mathcal{A}}_{J}} \mathbf{w}_{\beta \tilde{\phi}+\tilde{\psi}}\left(\left[a, a^{\prime}\right]_{J}\right) M_{\beta \tilde{\phi}+\tilde{\psi}}\left(\left[a, a^{\prime}\right]_{J},\left[c, c^{\prime}\right]_{K}\right) \\
& =\frac{1}{\rho_{\beta \tilde{\phi}+\tilde{\psi}}} \sum_{J ; a, a^{\prime} \in \overline{\mathcal{A}}_{J}} \mathbf{w}_{\beta \tilde{\phi}+\tilde{\psi}}\left(\left[a, a^{\prime}\right]_{J}\right) M_{\beta \tilde{\phi}+\tilde{\psi}}\left(a_{J}^{\prime}, c_{K}\right) \\
& =\frac{1}{\rho_{\beta \tilde{\phi}+\tilde{\psi}}} \sum_{J, \mathbf{e} \in \overline{\mathcal{A}}_{J}} \hat{\mathbf{w}}_{\beta \tilde{\phi}+\tilde{\psi}}\left(a_{J}^{\prime}\right) M_{\beta \tilde{\phi}+\tilde{\psi}}\left(a_{J}^{\prime}, c_{K}\right),
\end{aligned}
$$

for any $1 \leq K \leq N_{\phi}$ and $c, c^{\prime} \in \overline{\mathcal{A}}_{K}$. In this way we show that $\mathbf{w}_{\beta \tilde{\phi}+\tilde{\psi}}\left(\left[c, c^{\prime}\right]_{K}\right)$ does not depend on $c^{\prime}$. A similar computation shows that $\mathbf{v}_{\beta \tilde{\phi}+\tilde{\psi}}\left(\left[c, c^{\prime}\right]_{K}\right)$ does not depend on $c$, and we can write $\mathbf{w}_{\beta \tilde{\phi}+\tilde{\psi}}\left(c_{K}\right)$ instead of $\mathbf{w}_{\beta \tilde{\phi}+\tilde{\psi}}\left(\left[c, c^{\prime}\right]_{K}\right)$ and $\mathbf{v}_{\beta \tilde{\phi}+\tilde{\psi}}\left(c_{K}^{\prime}\right)$ instead of $\mathbf{v}_{\beta \tilde{\phi}+\tilde{\psi}}\left(\left[c, c^{\prime}\right]_{K}\right)$.

For each $J \in \mathcal{A}^{\prime}$ let $\overline{\mathbf{w}}_{\beta \tilde{\phi}+\tilde{\psi}}(J):=\sum_{a \in \overline{\mathcal{A}}_{J}} \mathbf{w}_{\beta \tilde{\phi}+\tilde{\psi}}\left(a_{J}\right)$ and $\overline{\mathbf{v}}_{\beta \tilde{\phi}+\tilde{\psi}}(J):=\sum_{a^{\prime} \in \overline{\mathcal{A}}_{J}} \mathbf{v}_{\beta \tilde{\phi}+\tilde{\psi}}\left(a_{J}^{\prime}\right)$. Then we have

$$
\begin{aligned}
\overline{\mathbf{w}}_{\beta \tilde{\phi}+\tilde{\psi}}(K) & =\frac{1}{\rho_{\beta \tilde{\phi}+\tilde{\psi}}} \sum_{J ; a, a^{\prime} \in \overline{\mathcal{A}}_{J} ; c \in \overline{\mathcal{A}}_{K}} \mathbf{w}_{\beta \tilde{\phi}+\tilde{\psi}}\left(\left[a, a^{\prime}\right]_{J}\right) M_{\beta \tilde{\phi}+\tilde{\psi}}\left(\left[a, a^{\prime}\right]_{J},\left[c, c^{\prime}\right]_{K}\right) \\
& =\frac{1}{\rho_{\beta \tilde{\phi}+\tilde{\psi}}} \sum_{J ; a, a^{\prime} \in \overline{\mathcal{A}}_{J} ; c \in \overline{\mathcal{A}}_{K}} \mathbf{w}_{\beta \tilde{\phi}+\tilde{\psi}}\left(a_{J}\right) M_{\beta \tilde{\phi}+\tilde{\psi}}\left(a_{J}^{\prime}, c_{K}\right) \\
& =\frac{1}{\rho_{\beta \tilde{\phi}+\tilde{\psi}}} \sum_{J ; a, a^{\prime} \in \overline{\mathcal{A}}_{J} ; c \in \overline{\mathcal{A}}_{K}} \overline{\mathbf{w}}_{\beta \tilde{\phi}+\tilde{\psi}}(J) \overline{\mathcal{M}}_{\beta \tilde{\phi}+\tilde{\psi}}(J, K),
\end{aligned}
$$

from which it follows $\rho_{\beta \tilde{\phi}+\tilde{\psi}} \in \operatorname{spec}\left(\overline{\mathcal{M}}_{\beta \tilde{\phi}+\tilde{\psi}}\right)$, with a left positive eigenvector $\overline{\mathbf{w}}_{\beta \tilde{\phi}+\tilde{\psi}}$. A similar computation shows that $\overline{\mathcal{M}}_{\beta \tilde{\phi}+\tilde{\psi}} \overline{\mathbf{v}}_{\beta \tilde{\phi}+\psi}=\rho_{\beta \tilde{\phi}+\tilde{\psi}} \overline{\mathbf{v}}_{\beta \tilde{\phi}+\psi}$. Corollary 1 in Appendix A ensures that $\rho_{\beta \tilde{\phi}+\tilde{\psi}}=\max \left(\operatorname{spec}\left(\overline{\mathcal{M}}_{\beta \tilde{\phi}+\tilde{\psi}}\right)\right)$.

Now, using the definition of the pull back measure $\bar{\mu}_{\beta \tilde{\phi}+\tilde{\psi}}:=\mu_{\beta \tilde{\phi}+\tilde{\psi}} \circ \pi^{-1}$, and taking into account (32) above, we have

$$
\begin{aligned}
\bar{\mu}_{\beta \tilde{\phi}+\tilde{\psi}}\left[J_{0} \cdots J_{n}\right] & =\sum_{a_{i}, a_{i}^{\prime} \in \overline{\mathcal{A}}_{J_{i}}} \mathbf{w}_{\beta \tilde{\phi}+\tilde{\psi}}\left(\left(a_{0}\right)_{J_{0}}\right) \frac{\prod_{i=0}^{n-1} M_{\beta \tilde{\phi}+\tilde{\psi}}\left(\left(a_{i}^{\prime}\right)_{J_{i}},\left(a_{i+1}\right)_{J_{i+1}}\right)}{\rho_{\beta \tilde{\phi}+\tilde{\psi}}^{n}} \mathbf{v}_{\beta \tilde{\phi}+\tilde{\psi}}\left(\left(a_{n}^{\prime}\right)_{J_{n}}\right) \\
& =\left(\sum_{a_{0} \in \overline{\mathcal{A}}_{J_{0}}} \mathbf{w}_{\beta \tilde{\phi}+\tilde{\psi}}\left(\left(a_{0}\right)_{J_{0}}\right)\right) \frac{\prod_{i=0}^{n-1}\left(\sum_{a_{i}^{\prime} \in \overline{\mathcal{A}}_{J_{i}}, a_{i+1} \in \overline{\mathcal{A}}_{J_{i+1}}} M_{\beta \tilde{\phi}+\tilde{\psi}}\left(\left(a_{i}^{\prime}\right)_{J_{i}},\left(a_{i+1}\right)_{J_{i+1}}\right)\right)}{\bar{\rho}_{\beta \tilde{\phi}+\tilde{\psi}}^{n}} \times\left(\sum_{a_{n}^{\prime} \in \overline{\mathcal{A}}_{J_{n}}} \mathbf{v}_{\beta \tilde{\phi}+\tilde{\psi}}\left(\left(a_{n}^{\prime}\right)_{J_{n}}\right)\right) \\
& =\overline{\mathbf{w}}_{\beta \tilde{\phi}+\tilde{\psi}}\left(J_{0}\right) \frac{\prod_{i=0}^{n-1} \overline{\mathcal{M}}_{\beta \tilde{\phi}+\tilde{\psi}}\left(J_{i}, J_{i+1}\right)}{\bar{\rho}_{\beta \tilde{\phi}+\tilde{\psi}}^{n}} \overline{\mathbf{v}}_{\beta \tilde{\phi}+\tilde{\psi}}\left(J_{n}\right),
\end{aligned}
$$

for each $n \in \mathbb{N}$ and $J_{0} \cdots J_{n} \in\left(\mathcal{A}^{\prime}\right)^{n+1}$, and the result follows. 


\section{Appendix A. Proof of Proposition 1 (Periodic Approximation)}

The proof of Proposition 1 is based upon the following Theorem, which is a slight adaptation of Corollary 6.2 in [4].

Theorem 2 (Perron-Frobenius: Primitive Case). Let $B$ be a finite alphabet, and $\mathcal{M}: B \times B \rightarrow \mathbb{R}^{+}$ a primitive matrix. Then there exists a unique $\rho \in \operatorname{spec}(\mathcal{M})$ such that $\rho:=\max |\operatorname{spec}(\mathcal{M})|$. Associated to $\rho$ there are right and left eigenvectors $\mathbf{v}, \mathbf{w}$, such that $\mathbf{w}^{\dagger} \mathbf{v}=1$. Furthermore, for every probability vector $\mathbf{x} \in(0,1)^{B}$, and for each $m \in \mathbb{N}$ we have

$$
\mathcal{M}^{m} \mathbf{x}=\rho^{m}\left(\mathbf{w}^{\dagger} \mathbf{x}\right) \mathbf{v} \exp \left( \pm \frac{\tau^{\lfloor m / \ell\rfloor} \ell d(\mathbf{x}, F \mathbf{x})}{1-\tau}\right)
$$

where

i) $\ell$ is the primitivity index of $\mathcal{M}$, i.e., the smallest integer such that $\mathcal{M}^{\ell}>0$,

ii) $d$ is the projective distance in the simplex $\Delta_{B}:=\left\{\mathbf{x} \in(0,1)^{B}:|\mathbf{x}|_{1}=1\right\}\left(|\cdot|_{1}\right.$ stands for the $\ell_{1}$ norm) of probability vectors,

$$
d(\mathbf{x}, \mathbf{y})=\log \left(\max _{b \in B}\{\mathbf{x}(b) / \mathbf{y}(b)\}\right)-\log \left(\min _{b \in B}\{\mathbf{x}(b) / \mathbf{y}(b)\}\right),
$$

iii) $F \mathbf{x}:=\mathcal{M} \mathbf{x} /|\mathcal{M} \mathbf{x}|_{1}$ is the action of the matrix $\mathcal{M}$ on the simplex $\Delta_{B}$, and

v) $\tau=(1-\Gamma) /(1+\Gamma)$ with

$$
\Gamma:=\sqrt{\min _{a, b, c, d \in B} \frac{\mathcal{M}^{\ell}(a, b) \mathcal{M}^{\ell}(c, d)}{\mathcal{M}^{\ell}(a, d) \mathcal{M}^{\ell}(c, b)}},
$$

is the Birkhoff coefficient of $\mathcal{M}^{\ell}$.

A rather direct consequence of the previous theorem is the following result.

Corollary 1 (Perron-Frobenius: Periodic Case). Let $B$ be a finite alphabet, and $\mathcal{M}: B \times B \rightarrow \mathbb{R}^{+}$ an irreducible matrix of period $p$. Let $B:=\bigsqcup_{i=0}^{p} B_{i}$ be the partition such that

$$
\mathcal{M}_{\psi}^{p}=\left(\begin{array}{ccccc}
\mathcal{M}_{0} & 0 & 0 & \cdots & 0 \\
0 & \mathcal{M}_{1} & 0 & \cdots & 0 \\
0 & 0 & \mathcal{M}_{2} & \cdots & 0 \\
\vdots & \vdots & \vdots & \ddots & \vdots \\
0 & 0 & 0 & \cdots & \mathcal{M}_{p-1}
\end{array}\right)
$$

with $\mathcal{M}_{i}: B_{i} \times B_{i} \rightarrow \mathbb{R}^{+}$primitive for each $0 \leq i<p$. Let $\mathbf{v}_{i}$ and $\mathbf{w}_{i}$ denote the unique left and right eigenvectors associated to the maximal eigenvalue $\rho_{i}:=\max \left|\operatorname{spec}\left(\mathcal{M}_{i}\right)\right|$, normalized such that $\mathbf{w}_{i}^{\dagger} \mathbf{v}_{i}=1$. Then, $\rho=\sqrt[p]{\rho_{i}}$ is the maximal eigenvalue of $\mathcal{M}$, with left and right eigenvectors $\mathbf{v}:=(1 / \sqrt{p}) \otimes_{i=0}^{p-1} \mathbf{v}_{i}$ and $\mathbf{w}:=(1 / \sqrt{p}) \otimes_{i=0}^{p-1} \mathbf{w}_{i}$ respectively. These are the unique left and right positive eigenvectors satisfying $\mathbf{w}^{\dagger} \mathbf{v}=1$. Furthermore, if we fix $0 \leq i<p$, and a probability vector 
$\mathbf{x}:=\otimes_{j=0}^{p-1} \mathbf{x}_{j}$, with $\mathbf{x}_{j} \in[0,1]^{B_{j}}$ such that $\mathbf{x}_{j}=0$ for $j \neq i$ and $\mathbf{x}_{i}>0$, then $\mathcal{M}^{k p+r} \mathbf{x}=\otimes_{j=0}^{p-1} \mathbf{y}_{j}$, with $\mathbf{y}_{j} \in[0,1]^{B_{j}}$ such that $\mathbf{y}_{j}=0$ for $j \neq i-r$ and

$$
\mathbf{y}_{i-r}=\frac{\rho^{m}}{p}\left(\mathbf{w}_{i}^{\dagger} \mathbf{x}_{i}\right) \mathbf{v}_{i-r} \exp \left( \pm \frac{\tau^{\lfloor k / \ell\rfloor} \ell d_{i}\left(\mathbf{x}_{i}, F_{i} \mathbf{x}_{i}\right)}{1-\tau}\right)
$$

where

i) $\ell$ is an upper bound for $\ell_{i}$, the primitivity index of the matrix $\mathcal{M}_{i}$,

ii) $d_{i}$ is the projective distance in $\Delta_{i}:=\left\{\mathbf{x}_{i} \in(0,1)^{B_{i}}:\left|\mathbf{x}_{i}\right|_{1}=1\right\}$,

iii) $F_{i} \mathbf{x}_{i}:=\mathcal{M}_{i} \mathbf{x}_{B_{i}} /\left|\mathcal{M}_{i} \mathbf{x}_{i}\right|_{1}$ is the action of the matrix $\mathcal{M}^{p}$ on $\Delta_{i}$, and

iv) $\tau$ is an upper bound for the Birkhoff coefficient $\tau_{i}:=\left(1-\Gamma_{i}\right) /\left(1+\Gamma_{i}\right)$, with

$$
\Gamma_{i}:=\sqrt{\min _{a, b, c, d \in B_{i}} \frac{\mathcal{M}_{i}^{\ell}(a, b) \mathcal{M}_{i}^{\ell}(c, d)}{\mathcal{M}_{i}^{\ell}(a, d) \mathcal{M}^{\ell}(c, b)}} .
$$

\section{Proof of Proposition 1}

Let $\mathcal{M}:=\mathcal{M}_{\beta \phi+\psi}, \rho:=\rho_{\beta \phi+\psi}$, and for each $0 \leq i<\bar{p}$, let $\mathcal{M}_{i}:=\mathcal{M}_{\beta \phi+\psi, i}, \mathbf{v}_{i}:=\mathbf{v}_{\beta \phi+\psi, i}$, and $\mathbf{w}_{i}:=\mathbf{w}_{\beta \phi+\psi, i}$, as defined in Subsection 8.1.1. Let $\ell$ be the maximum of the primitivity indices of the matrices $\mathcal{M}_{i}, 0 \leq i<\bar{p}$, and denote by $i(a)$ the index of the set $\mathcal{A}_{i}$ containing $a$. Applying Corollary 1 and using (20), we obtain

$$
\begin{aligned}
& \mathcal{P}_{\beta \phi+\psi}^{(k \bar{p})}\left[\mathbf{b}_{0}^{n}\right]=\prod_{i=0}^{n-1} \mathcal{M}\left(\mathbf{b}_{i}, \mathbf{b}_{i+1}\right) \frac{\delta_{\mathbf{b}_{n}}^{\dagger} \mathcal{M}^{k \bar{p}-n} \delta_{\mathbf{b}_{0}}}{\sum_{b \in B} \delta_{b}^{\dagger} \mathcal{M}^{k \bar{p}} \delta_{b}} \\
& =\frac{\prod_{i=0}^{n-1} \mathcal{M}\left(\mathbf{b}_{i}, \mathbf{b}_{i+1}\right)}{\rho^{n}} \frac{\left(\delta_{\mathbf{b}_{n}}^{\dagger} \mathcal{M}_{i\left(\mathbf{b}_{n}\right)}^{\ell}\right)^{\dagger} \mathbf{v}_{i\left(\mathbf{b}_{n}\right)} \mathbf{w}_{i\left(\mathbf{b}_{0}\right)}^{\dagger}\left(\mathcal{M}^{\ell} \delta_{\mathbf{b}_{0}}\right)}{\sum_{b \in B}\left(\delta_{b}^{\dagger} \mathcal{M}^{\ell}\right) \mathbf{v}_{i(b)} \mathbf{w}_{i(b)}^{\dagger}\left(\mathcal{M}^{\ell} \delta_{b}\right)} \\
& \times \exp \left( \pm \frac{C \tau^{\left\lfloor\frac{k-n / \bar{p}}{\ell}\right\rfloor-2}}{1-\tau}\right) \\
& =\frac{\mathbf{w}_{i\left(\mathbf{b}_{0}\right)}\left(\mathbf{b}_{0}\right)\left(\prod_{i=0}^{n-1} \mathcal{M}\left(\mathbf{b}_{i}, \mathbf{b}_{i+1}\right)\right) \mathbf{v}_{i\left(\mathbf{b}_{n}\right)}\left(\mathbf{b}_{n}\right)}{\rho^{n} \sum_{b \in B} \mathbf{v}_{i(b)}(\mathbf{b}) \mathbf{w}_{i(b)}(b)} \exp \left( \pm \frac{C \tau^{\left\lfloor\frac{k-n / \bar{p}}{\ell}\right\rfloor-2}}{1-\tau}\right) \\
& =\frac{\mathbf{w}_{i\left(\mathbf{b}_{0}\right)}\left(\mathbf{b}_{0}\right)\left(\prod_{i=0}^{n-1} \mathcal{M}\left(\mathbf{b}_{i}, \mathbf{b}_{i+1}\right)\right) \mathbf{v}_{i\left(\mathbf{b}_{n}\right)}\left(\mathbf{b}_{n}\right)}{p \rho^{n}} \exp \left( \pm \frac{C \tau^{\left\lfloor\frac{k-n / \bar{p}}{\ell}\right\rfloor-2}}{1-\tau}\right) \\
& =\frac{\mathbf{w}\left(\mathbf{b}_{0}\right)\left(\prod_{i=0}^{n-1} \mathcal{M}\left(\mathbf{b}_{i}, \mathbf{b}_{i+1}\right)\right) \mathbf{v}\left(\mathbf{b}_{n}\right)}{\rho^{n}} \exp \left( \pm \frac{C \tau^{\left\lfloor\frac{k-n / \bar{p}}{\ell}\right\rfloor-2}}{1-\tau}\right) \\
& =\mu_{\beta \phi+\psi}\left[\mathbf{b}_{0}^{n}\right] \exp \left( \pm \frac{C \tau^{\left\lfloor\frac{k-n / \bar{p}}{\ell}\right\rfloor-2}}{1-\tau}\right),
\end{aligned}
$$

where $C:=2 \ell \max _{0 \leq i<\bar{p}} \max _{b \in B_{i}} d_{i}\left(\mathcal{M}_{i}^{\ell+1} \delta_{b}, \mathcal{M}_{i}^{\ell} \delta_{b}\right)$, where $\delta_{b}: B_{i} \rightarrow \mathbb{R}$ is the unit vector in the direction of $b$, and where $\tau=\max _{0 \leq i<\bar{p}} \tau_{i}$, where $\tau_{i}$ is the Birkhoff coefficient of $\mathcal{M}_{i}^{\ell}$. 
Therefore we have

$$
\mu_{\beta \phi+\psi}\left[\mathbf{b}_{0}^{n}\right]=\mathcal{P}_{\beta \phi+\psi}^{(k \bar{p})}\left[\mathbf{b}_{0}^{n}\right] \exp \left( \pm \frac{C \tau^{\left\lfloor\frac{k-n / \bar{p}}{\ell}\right\rfloor-2}}{1-\tau}\right)
$$

It remains to bound $\tau$ and $C$ to conclude the proof of the proposition. Using the fact that $\tau \leq$ $1-\min _{0 \leq i<\bar{p}} \Gamma_{i}$, with $\Gamma_{i}$ as in the statement of Corollary 1, we obtain

$$
\begin{aligned}
& \tau \leq 1-\min _{0 \leq i<\bar{p}} \min _{a, b, d \in \mathcal{A}_{i}} \frac{\mathcal{M}_{i}^{\ell}(a, b)}{\mathcal{M}_{i}^{\ell}(a, d)}=1-\min _{a, b, d \in \mathcal{A}} \frac{\mathcal{M}_{i}^{\ell}(a, b)}{\mathcal{M}_{i}^{\ell}(a, d)}
\end{aligned}
$$

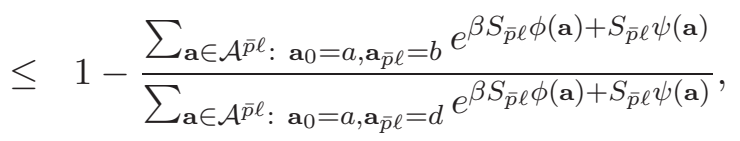

$$
\begin{aligned}
& \leq 1-\frac{e^{\left.-\bar{p} \ell\left(\beta\|\phi\|_{\infty}+\|\psi\|_{\infty}\right)+\log (\# \mathcal{A})\right)}}{e^{\bar{p} \ell\left(\beta\|\phi\|_{\infty}+\|\psi\|_{\infty}+\log (\# \mathcal{A})\right)}} \\
& \leq 1-\exp \left(-2 \bar{p} \ell\left(\beta\|\phi\|_{\infty}+\|\psi\|_{\infty}+\log (\# \mathcal{A})\right)\right) .
\end{aligned}
$$

Now, for each $0 \leq i<\bar{p}$ and $b \in \mathcal{A}_{i}$ we have

$$
\begin{aligned}
& d_{i}\left(\mathcal{M}_{i}^{\ell+1} \delta_{b}, \mathcal{M}_{i}^{\ell} \delta_{b}\right)=\max _{a, a^{\prime} \in \mathcal{A}_{i}} \log \left(\frac{\sum_{\substack{\mathbf{a} \in \mathcal{A}_{\bar{p}}(\ell+1) \\
\mathbf{a}_{0}=a, \mathbf{a}_{\bar{p}(\ell+1)}=b}} e^{S_{\bar{p}(\ell+1)}(\beta \phi+\psi)(\mathbf{a})} \sum_{\substack{\mathbf{a} \in \mathcal{A}_{\bar{p} \ell} \ell \\
\mathbf{a}_{0}=a^{\prime}, \mathbf{a}_{\bar{p} \ell}=b}} e^{S_{\bar{p} \ell}(\beta \phi+\psi)(\mathbf{a})}}{\sum_{\substack{\mathbf{a} \in \mathcal{p}(\ell+1) \\
\mathbf{a}_{0}=a^{\prime}, \mathbf{a}_{\bar{p}(\ell+1)}=b}} e^{S_{\bar{p}(\ell+1)}(\beta \phi+\psi)(\mathbf{a})} \sum_{\substack{\mathbf{a} \in \mathcal{A} \bar{p} \ell \\
\mathbf{a}_{0}=a, \mathbf{a}_{\bar{p} \ell}=b}} e^{S_{\bar{p} \ell}(\beta \phi+\psi)(\mathbf{a})}}\right) \\
& \leq \log \left(\frac{e^{\bar{p}(2 \ell+1)\left(\beta\|\phi\|_{\infty}+\|\psi\|_{\infty}+\log (\# \mathcal{A})\right)}}{e^{\left.-\bar{p}(2 \ell+1)\left(\beta\|\phi\|_{\infty}+\|\psi\|_{\infty}\right) \log (\# \mathcal{A})\right)}}\right) \\
& \leq 2 \bar{p}(2 \ell+1)\left(\beta\|\phi\|_{\infty}+\|\psi\|_{\infty}+\log (\# \mathcal{A})\right),
\end{aligned}
$$

where $\|\cdot\|$ denotes the supremum norm. With these two bounds, and taking into account (33), we obtain

$$
\mu_{\beta \phi+\psi}\left[\mathbf{a}_{0}^{n}\right]=\mathcal{P}_{\beta \phi+\psi}^{(k \bar{p})}\left[\mathbf{a}_{0}^{n}\right] \exp \left( \pm\left(\beta n_{\phi}+n_{\psi}\right) e^{\beta s_{\phi}+s_{\psi}}\left(1-e^{-\left(\beta s_{\phi}+s_{\psi}\right)}\right)^{\frac{k}{\ell}-\frac{n}{\bar{p} \ell}-3}\right)
$$

for some positive constants $s_{\phi}, s_{\psi}, n_{\phi}$ and $n_{\psi}$. Since $k \bar{p}>e^{\gamma \beta}+n$ and since by assumption $\gamma>s_{\phi}$, we have

$$
\frac{k}{\ell}-\frac{n}{\bar{p} \ell}-3 \geq 2 \beta\left(\gamma-s_{\phi}\right) e^{\beta s_{\phi}+s_{\psi}} \text { for } \beta \text { large enough. }
$$

Taking into account that $1-e^{-\left(\beta s_{\phi}+s_{\psi}\right)} \leq \exp \left(-e^{-\left(\beta s_{\phi}+s_{\psi}\right)}\right)$, we finally obtain, for $\beta$ large enough,

$$
\mu_{\beta \phi+\psi}\left[\mathbf{b}_{0}^{n}\right]=\mathcal{P}_{\beta \phi+\psi}^{(k \bar{p})}\left[\mathbf{b}_{0}^{n}\right] \exp \left( \pm\left(\beta n_{\phi}+n_{\psi}\right) e^{-2 \beta\left(\gamma-s_{\phi}\right)}\right)=\mathcal{P}_{\beta \phi+\psi}^{(k \bar{p})}\left[\mathbf{b}_{0}^{n}\right] \exp \left( \pm e^{-\beta\left(\gamma-s_{\phi}\right)}\right)
$$

for all $\mathbf{b}_{0}^{n}$. The proof of Proposition 1 is now finished. 
Remark 2. We have $s_{\phi}=2 \bar{p} \ell\|\phi\|_{\infty}, s_{\psi}=2 \bar{p} \ell\left(\|\psi\|_{\infty}+\log (\# \mathcal{A})\right)$, and $n_{*}:=2(2 \ell+1) s_{*}$ where $*=\phi, \psi$.

\section{Appendix B. Auxiliary Inequalities of Lemma 1}

\section{B.1. Incursion Length.}

Proposition 2 (The Incursion Time is Exponential). Let us suppose $\left[\mathbf{b}_{0}^{n}\right] \cap \bar{X}_{J} \neq \emptyset$ for some $1 \leq J \leq N_{\phi}$. If $q=\left\lfloor e^{\eta \beta}\right\rfloor<p$, with $-\phi_{g} / 4<\eta<-\phi_{g} / 3$, then we have

$$
\sum_{\mathbf{c} \in \operatorname{Per}_{p}(X) \cap\left[\mathbf{b}_{0}^{n}\right]} e^{S_{p}(\beta \phi+\psi)(\mathbf{c})}=\exp \left( \pm 2 e^{\beta \frac{\phi_{g}}{4}}\right) \sum_{\mathbf{c} \in X^{(p, q)} \cap\left[\mathbf{b}_{0}^{n}\right]} e^{S_{p}(\beta \phi+\psi)(\mathbf{c})},
$$

for $\beta$ large enough.

Proof. We obviously have

$$
\sum_{\mathbf{c} \in \operatorname{Per}_{p}(X) \cap\left[\mathbf{b}_{0}^{n}\right]} e^{S_{p}(\beta \phi+\psi)(\mathbf{c})} \geq \exp \left(-2 e^{\beta \frac{\phi_{g}}{4}}\right) \sum_{\mathbf{c} \in X^{(p, q)} \cap\left[\mathbf{b}_{0}^{n}\right]} e^{S_{p}(\beta \phi+\psi)(\mathbf{c})} .
$$

For each $a$ and $a^{\prime} \in \mathcal{A}$, let us denote by $a \circlearrowleft a^{\prime}$ a fixed circuit in $\mathcal{G}_{X}$ of period $p_{0}=p_{0}\left(a, a^{\prime}\right)$, containing both $a$ and $a^{\prime}$. By choosing $p_{0}$ the minimal integer for which such circuit exists, we ensure that $p_{0}\left(a, a^{\prime}\right) \leq 2 \# \mathcal{A}$. Let us denote by $a \rightarrow a^{\prime}$ and $a^{\prime} \rightarrow a$ the path segments composing $a \circlearrowleft a^{\prime}$, and by $a \rightarrow a^{\prime}$ and $a^{\prime} \rightarrow a$ the corresponding $X$-admissible word. Let us also denote by $a \circlearrowleft a^{\prime}$ the periodic point in $\operatorname{Per}_{p_{0}}(X) \cap[a]$ defined by the circuit $a \circlearrowleft a^{\prime}$.

To each periodic orbit in $\mathbf{a} \in \operatorname{Per}_{p}(X) \cap\left[\mathbf{b}_{0}^{n}\right]$ such that $\mathbf{a}_{p-q^{2}-1}=a^{\prime}$ and $\mathbf{a}_{q^{2}}=a$, we associate the periodic points

$$
\begin{aligned}
& \mathbf{a}_{\text {int }}:=\left(\mathbf{a}_{0}^{q^{2}-1} a \rightarrow a^{\prime} \mathbf{a}_{p-q^{2}}^{p-1}\right.)^{\infty} \in \operatorname{Per}_{p_{1}}(X) \cap \sigma^{-q^{2}}\left[a \rightarrow a^{\prime}\right], \\
& \mathbf{a}_{\mathrm{ext}}:=\left(a^{\prime} \rightarrow a \mathbf{a}_{q^{2}+1}^{p-q^{2}-2}\right)^{\infty} \in \operatorname{Per}_{p_{2}}(X) \cap\left[a^{\prime} \rightarrow a\right],
\end{aligned}
$$

with $p_{1}=p_{1}\left(a, a^{\prime}\right):=2 q^{2}+\left|a \rightarrow a^{\prime}\right|$ and $p_{2}:=p_{2}\left(a, a^{\prime}\right)=p-2 q^{2}-2+\left|a^{\prime} \rightarrow a\right|$. Using this notation we can write,

$$
\sum_{\mathbf{a} \in \operatorname{Per}_{p}(X) \cap\left[\mathbf{b}_{0}^{n}\right]} e^{S_{p}(\beta \phi+\psi)(\mathbf{a})}=\sum_{a, a^{\prime} \in \mathcal{A}} e^{-S_{p_{0}}(\beta \phi+\psi)\left(a \circlearrowleft a^{\prime}\right)} e^{S_{p_{1}}(\beta \phi+\psi)\left(\mathbf{a}_{\text {int }}\right)} e^{S_{p_{2}}(\beta \phi+\psi)\left(\mathbf{a}_{\mathrm{ext}}\right)},
$$

from which it follows

$$
\sum_{\mathbf{a} \in \operatorname{Per}_{p}(X) \cap\left[\mathbf{b}_{0}^{n}\right]} e^{S_{p}(\beta \phi+\psi)(\mathbf{c})}=\sum_{a, a^{\prime} \in \mathcal{A}} e^{-S_{p_{0}}(\beta \phi+\psi)\left(a \circlearrowleft a^{\prime}\right)} \sum_{\mathbf{c} \in \operatorname{Per}_{p_{2}}(X) \cap\left[a^{\prime} \rightarrow a\right]} e^{S_{p_{2}}(\beta \phi+\psi)(\mathbf{a})}
$$

Let us now study the interior sums $\sum_{\mathbf{a} \in \operatorname{Per}_{p_{1}}(X) \cap\left[\mathbf{b}_{0}^{n}\right] \cap \sigma^{-q^{2}\left[a \rightarrow a^{\prime}\right]}} e^{S_{p_{1}}(\beta \phi+\psi)(\mathbf{a})}$. 
Each periodic point $\mathbf{a} \in \operatorname{Per}_{p_{1}}(X) \cap\left[\mathbf{b}_{0}^{n}\right] \cap \sigma^{-q^{2}}\left[a \rightarrow a^{\prime}\right]$ defines a circuit $C(\mathbf{a})$ in $\mathcal{G}_{X}$. We decompose this circuit into its incursion-excursion path segments,

$$
C(\mathbf{a}):=a_{1} \rightsquigarrow a_{1}^{\prime} \cdot b_{1} \rightsquigarrow b_{1}^{\prime} \cdots a_{\kappa} \rightsquigarrow a_{\kappa}^{\prime} \cdot b_{\kappa} \rightsquigarrow b_{\kappa}^{\prime},
$$

which are defined as follows:

a) the segment $a_{1} \rightsquigarrow a_{1}^{\prime}$ lies on $\mathcal{G}_{J} \subset \mathcal{G}_{X}$, the digraph associated to the heavy component $\bar{X}_{J}$ such that $\left[\mathbf{b}_{0}^{n}\right] \cap \bar{X}_{J} \neq \emptyset$;

b) none of the paths $b_{i} \rightsquigarrow b_{i}^{\prime}$, for $1 \leq i \leq \kappa=\kappa(\mathbf{a})$, includes arrows from the digraph $\mathcal{G}_{\bar{X}}$ defining $\bar{X}$

c) each path $a_{i} \rightsquigarrow a_{i}^{\prime}$, for $1 \leq i \leq \kappa$, lies on $\mathcal{G}_{\bar{X}}$.

If the end vertices $a_{i}^{\prime}$ and $b_{i}$ do not coincide, the concatenation $a_{i}^{\prime} \cdot b_{i}$ is made by an arrow in $\mathcal{G}_{X}$. The same holds for the end vertices $b_{i}^{\prime}$ and $a_{i+1}$.

We extend $C(\mathbf{a})$ by adding, for each $1 \leq i \leq \kappa$, a circuit $a_{i} \circlearrowleft a_{i}^{\prime}$ lying on the same transitive component of $\bar{X}$ as $a_{i}$, containing both $a_{i}$ and $a_{i}^{\prime}$, and having minimal length. Since all the added circuits lie in $\mathcal{G}_{\bar{X}}$, then the extended circuit

$$
\begin{aligned}
& C_{\text {ext }}(\mathbf{a}):=a_{1} \rightsquigarrow a_{1}^{\prime} \cdot b_{1} \rightsquigarrow b_{1}^{\prime} \cdots a_{\kappa} \rightsquigarrow a_{\kappa}^{\prime} \cdot b_{\kappa} \rightsquigarrow b_{\kappa}^{\prime} \\
& \begin{array}{ll}
\circlearrowleft & \circlearrowleft \\
a_{i} & a_{\kappa}
\end{array}
\end{aligned}
$$

is such that $\phi(C(\mathbf{a}))=\phi\left(C_{\text {ext }}(\mathbf{a})\right)$. Also, since all the added circuits have minimal length, then $\psi(C(\mathbf{a})) \leq \psi\left(C_{\text {ext }}(\mathbf{a})\right)+2 \kappa \# \mathcal{A}\|\psi\|_{\infty}$.

We reorganize the path segment in $C_{\text {ext }}(\mathbf{a})$ in order to obtain

$$
C_{\text {ext }}(\mathbf{a})=\sum_{i=1}^{\kappa} a_{i} \rightsquigarrow a_{i}^{\prime}+a_{1} \rightarrow a_{1}^{\prime} \cdot b_{1} \rightsquigarrow b_{1}^{\prime} \cdots a_{i} \rightarrow a_{i}^{\prime} \cdot b_{i} \rightsquigarrow b_{i}^{\prime} \cdots a_{\kappa} \rightarrow a_{\kappa}^{\prime} \cdot b_{\kappa} \rightsquigarrow b_{\kappa}^{\prime},
$$

where, for each $1 \leq i \leq \kappa$, the circuit $a \stackrel{\curvearrowleft}{\rightsquigarrow} a^{\prime}$ is obtained by concatenation of $a \rightsquigarrow a^{\prime}$ and the path segment $a^{\prime} \rightarrow a$ of the added circuit $a \circlearrowleft a^{\prime}$. For the complementary circuit

$$
C^{\prime}(\mathbf{a}):=a_{1} \rightarrow a_{1}^{\prime} \cdot b_{1} \rightsquigarrow b_{1}^{\prime} \cdots a_{i} \rightarrow a_{i}^{\prime} \cdot b_{i} \rightsquigarrow b_{i}^{\prime} \cdots a_{\kappa} \rightarrow a_{\kappa}^{\prime} \cdot b_{\kappa} \rightsquigarrow b_{\kappa}^{\prime},
$$

we replace the segments $a_{i} \rightsquigarrow a_{i}^{\prime}$ in $C(\mathbf{a})$, by the paths of minimal length $a_{i} \rightarrow a_{i}^{\prime}$ appearing in $a_{i} \circlearrowleft a_{i}^{\prime}$. We use the same convention for the concatenations $a_{i}^{\prime} \cdot b_{i}$ and $b_{i}^{\prime} \cdot a_{i+1}$ as above. All the circuits $a_{i} \cong a_{i}^{\prime}$ lie in $\mathcal{G}_{\bar{X}}$, therefore they maximize $\phi$. Notice also that the complementary circuit $C^{\prime}(\mathbf{a})$ does not include any circuit maximizing $\phi$, therefore $\phi\left(C^{\prime}(\mathbf{a})\right) \leq\left|C^{\prime}(\mathbf{a})\right| \phi_{g}$.

We can bound from above the sum of the potentials $\phi$ and $\psi$ on the circuit $C(\mathbf{a})$ by the same sums over the extended circuit $C_{\text {ext }}(\mathbf{a})$ as follows:

$$
\begin{aligned}
\phi(C(\mathbf{a})) & =\phi\left(C_{\text {ext }}(\mathbf{a})\right)=\phi\left(C^{\prime}(\mathbf{a})\right) \leq\left|C^{\prime}(\mathbf{a})\right| \phi_{g} \\
\psi(C(\mathbf{a})) & \leq \psi\left(C_{\text {ext }}(\mathbf{a})\right)+2 \kappa \# \mathcal{A}\|\psi\|_{\infty}=\sum_{i=1}^{\kappa} \psi\left(a_{i} \cong a_{i}^{\prime}\right)+\psi\left(C^{\prime}(\mathbf{a})\right)+2 \kappa \# \mathcal{A}\|\psi\|_{\infty} \\
& \leq \sum_{i=1}^{\kappa} \psi\left(a_{i} \curvearrowleft a_{i}^{\prime}\right)+(2 \# \mathcal{A}+1)\|\psi\|_{\infty}\left|C^{\prime}(\mathbf{a})\right|,
\end{aligned}
$$

where we use the fact that $\kappa \leq\left|C^{\prime}(\mathbf{a})\right|$. 
We can group the periodic points in $\mathcal{P}_{1}:=\operatorname{Per}_{p_{1}}(X) \cap\left[\mathbf{b}_{0}^{n}\right] \cap \sigma^{-q^{2}}\left[a \rightarrow a^{\prime}\right]$ according to the number of excursion paths they contain. By doing so we have

$$
\begin{aligned}
\sum_{\mathbf{a} \in \mathcal{P}_{1}} e^{S_{p_{1}}(\beta \phi+\psi)(\mathbf{a})} & \leq \sum_{\kappa=0}^{\left\lfloor p_{1} / 2\right\rfloor} \sum_{\substack{\mathbf{a} \in \mathcal{P}_{1} \\
\kappa(\mathbf{a})=\kappa}} e^{S_{p_{1}}(\beta \phi+\psi)(\mathbf{a})} \\
& \leq \sum_{\kappa=0}^{\left\lfloor p_{1} / 2\right\rfloor} \sum_{\substack{\mathbf{a} \in \mathcal{P}_{1} \\
\kappa(\mathbf{a})=\kappa}} e^{\left(\beta \phi_{g}+(2 \# \mathcal{A}+1)\|\psi\|_{\infty}\right)\left|C^{\prime}(\mathbf{a})\right|} \prod_{i=1}^{\kappa} e^{\psi\left(a_{i} \cong a_{i}^{\prime}\right)}
\end{aligned}
$$

Now we group all the periodic points in $\left\{\mathbf{a} \in \mathcal{P}_{1}: \kappa(\mathbf{a})=\kappa\right\}$ in classes defined by the total total length of the complementary circuit, $L(\mathbf{a}):=\left|C^{\prime}(\mathbf{a})\right|$, the lengths of the incursion and the excursion segments, $m_{i}:=\left|a_{i} \rightsquigarrow a_{i}^{\prime}\right|$ and $n_{i}:=\left|b_{i} \rightsquigarrow b_{i}^{\prime}\right|$ respectively, and the location of the origin inside the first incursion $a_{1} \rightsquigarrow a_{1}^{\prime}$. Taking into account Corollary 1 and the fact that $P(\psi \mid \bar{X})=P\left(\psi \mid \bar{X}_{J}\right)$, for $J=1 \ldots N_{\phi}$, we obtain

$$
\begin{aligned}
& \sum_{\mathbf{a} \in \mathcal{P}_{1}} e^{S_{p_{1}}(\beta \phi+\psi)(\mathbf{a})} \leq \sum_{\substack{\mathbf{a} \in \mathcal{P}_{1} \\
L(\mathbf{a})<L_{0}}} e^{S_{p_{1}}(\beta \phi+\psi)(\mathbf{a})}+\sum_{\kappa=0}^{\lfloor L / 2\rfloor} \sum_{L=L_{0}}^{p_{1}} e^{\left(\beta \phi_{g}+(2 \# \mathcal{A}+1)\|\psi\|_{\infty}+\log (\# \mathcal{A})\right) L} \\
& \times \sum_{\substack{\sum m_{i}=p_{1}-L \\
\sum n_{i}=L}} m_{1} K_{\psi} e^{S_{n} \psi\left(\mathbf{b}_{0}^{n}\right)} \mathcal{M}_{\psi, J}^{m_{1}-n}\left(\mathbf{b}_{n}, \mathbf{b}_{0}\right) \prod_{i=2}^{\kappa} K_{\psi} \operatorname{tr}\left(\mathcal{M}_{\psi, J}^{m_{i}}\right),
\end{aligned}
$$

for all $\beta \geq\left((2 \# \mathcal{A}+1)\|\psi\|_{\infty}+\log (\# \mathcal{A})\right) /\left|\phi_{g}\right|$. The integer $L_{0} \geq 2$ will be fixed later on. Here we bound the sums of factors $e^{\psi\left(a_{i} \cong a_{i}^{\prime}\right)}$ by a constant multiple of $\operatorname{tr}\left(\mathcal{M}_{\psi, J}^{m_{i}}\right)$, except for the term with $i=1$, which we bound by a constant factor of $e^{S_{n} \psi\left(\mathbf{b}_{0}^{n}\right)} \mathcal{M}_{\psi, J}^{m_{1}-n}\left(\mathbf{b}_{n}, \mathbf{b}_{0}\right)$. This last bound follows from the fact that $a_{1} \cong a_{1}^{\prime}$ includes the path segment $\left(\mathbf{b}_{0}, \mathbf{b}_{1}, \ldots, \mathbf{b}_{n}\right)$. The constant $K_{\psi} \geq \# \mathcal{A}$ is taken large enough to include the counting of transitive components in $\bar{X}$, and to compensate the differences in trace among transitive components and the difference between $\left|a_{i} \rightsquigarrow a_{i}^{\prime}\right|$ and $m_{i}$ for each $1 \leq i \leq \kappa$. The factor $m_{1}$ takes into account the all the possible locations of the origin with inside the first incursion. We are also considering the fact that $2 \kappa(\mathbf{a}) \leq L(\mathbf{a})$.

Now, the normalization $P(\psi \mid \bar{X})=0$ ensures that

$$
\begin{aligned}
\sum_{\substack{\mathbf{a} \in \mathcal{P}_{1} \\
L(\mathbf{a}) \geq L_{0}}} e^{S_{p_{1}}(\beta \phi+\psi)(\mathbf{a})} & \leq e^{S_{n} \psi\left(\mathbf{b}_{0}^{n}\right)} \sum_{L=L_{0}}^{p_{1}} e^{\left(\beta \phi_{g}+(2 \# \mathcal{A}+1)\|\psi\|_{\infty}+\log (\# \mathcal{A}) L\right.} p_{1} \sum_{\kappa=0}^{\lfloor L / 2\rfloor}\left(\begin{array}{c}
p_{1} \\
2 \kappa-1
\end{array}\right) D_{\psi}^{\kappa} \\
& \leq e^{S_{n} \psi\left(\mathbf{b}_{0}^{n}\right)} \sum_{L=L_{0}}^{p_{1}} e^{\left(\beta \phi_{g}+(2 \# \mathcal{A}+1)\|\psi\|_{\infty}+\log (\# \mathcal{A})+\log \left(p_{1}\right)+\log \left(D_{\psi}\right)+\log (L) / L\right) L} .
\end{aligned}
$$


The constant $D_{\psi} \geq K_{\psi}$ includes an upper bound for the factors $\operatorname{tr}\left(\mathcal{M}_{\phi, J}^{m}\right)$. Since $p_{1} \leq 2 e^{2 \beta \eta}+\mid a \rightarrow$ $a^{\prime} \mid$, with $\eta<-\phi_{g} / 3$, it follows from the previous inequality that

$$
\begin{aligned}
\sum_{\substack{\mathbf{a} \in \mathcal{P}_{1} \\
L(\mathbf{a}) \geq L_{0}}} e^{S_{p_{1}}(\beta \phi+\psi)(\mathbf{a})} & \leq e^{S_{n} \psi\left(\mathbf{b}_{0}^{n}\right)} \sum_{L=L_{0}}^{p_{1}} e^{\left(\beta \phi_{g}+(2 \# \mathcal{A}+1)\|\psi\|_{\infty}+\log (\# \mathcal{A})+\log \left(p_{1}\right)+\log \left(D_{\psi}\right)+e^{-1}\right) L} \\
& \leq 2 e^{S_{n} \psi\left(\mathbf{b}_{0}^{n}\right)} e^{\beta L_{0} \frac{\phi_{g}}{3}}
\end{aligned}
$$

for all $\beta$ greater than a convenient $\beta\left(L_{0}\right)$.

Let $k_{1}:=\min \left\{k \in \mathbb{N}: \operatorname{Per}_{k p_{J}}\left(\bar{X}_{J}\right) \cap\left[\mathbf{a}_{p-q^{2}}^{q^{2}-1} \neq \emptyset, \forall \mathbf{a}_{p-q^{2}}^{q^{2}-1} \bar{X}_{J}\right.\right.$-admissible with $\left.\mathbf{a}_{0}^{n}=\mathbf{b}_{0}^{n}\right\}$. It is not hard to verify that such a minimum exists. To each $\mathbf{a} \in \operatorname{Per}_{p_{1}}\left(\bar{X}_{J}\right) \cap\left[\mathbf{b}_{0}^{n}\right]$ such that $\mathbf{a}_{p-q^{2}}^{q^{2}-1}$ is $\bar{X}_{J}$-admissible, we associate a fixed periodic point $\mathbf{a}_{\text {int }} \in \operatorname{Per}_{k p_{J}}\left(\bar{X}_{J}\right) \cap\left[\mathbf{b}_{0}^{n}\right] \cap \sigma^{q^{2}}\left[\mathbf{a}_{p-q^{2}}^{q^{2}}\right]$, and the periodic point $a \circlearrowleft a^{\prime} \in \operatorname{Per}_{p_{0}}(X)$. Clearly $S_{p_{1}} \phi(\mathbf{a})=S_{p_{0}} \phi\left(a \circlearrowleft a^{\prime}\right) \geq-2 \# \mathcal{A}\|\psi\|_{\infty}$ and $S_{p_{1}} \psi(\mathbf{a}) \geq$ $S_{k_{1} p_{J}} \psi\left(\mathbf{a}_{\text {int }}\right)-2 \# \mathcal{A}\|\psi\|_{\infty}$. From this, and taking into account Corollary 1, it follows that

$$
\begin{aligned}
& \sum_{\substack{\mathbf{a} \in \mathcal{P}_{1} \\
\left[\mathbf{a}^{\mathbf{a}^{2}-1} p^{2}\right] \cap \bar{X}_{J} \neq \emptyset}} e^{S_{p_{1}}(\beta \phi+\psi)(\mathbf{a})} \geq e^{-2 \# \mathcal{A}\left(\beta\|\phi\|_{\infty}+\|\psi\|_{\infty}\right)} \# \mathcal{A}^{2 q^{2}-k_{1} p_{J}} \sum_{\mathbf{a} \in \operatorname{Per}_{k_{1} p_{J}}\left(\bar{X}_{J}\right) \cap\left[\mathbf{b}_{0}^{n}\right] \neq \emptyset} e^{S_{k_{1} p_{J}} \psi(\mathbf{a})} \\
& \geq e^{-2 \# \mathcal{A}\left(\beta\|\phi\|_{\infty}+\|\psi\|_{\infty}\right)} \# \mathcal{A}^{2 q^{2}-k_{1} p_{J}} e^{S_{n} \phi\left(\mathbf{b}_{0}^{n}\right)} \mathcal{M}_{\psi, J}^{k_{1} p_{J}-n}\left(\mathbf{b}_{n}, \mathbf{b}_{0}\right) \\
& \geq B e^{S_{n} \psi\left(\mathbf{b}_{0}^{n}\right)} e^{-2 \# \mathcal{A}\left(\beta\|\phi\|_{\infty}+\|\psi\|_{\infty}\right)}
\end{aligned}
$$

with $B=B(\psi):=\# \mathcal{A}^{2 q^{2}-k_{1} p_{J}} \mathbf{v}_{\psi, J}\left(\mathbf{b}_{n}\right) \mathbf{w}_{\psi, J}\left(\mathbf{b}_{0}\right) e^{-C_{\psi}}$. This and (35) imply

$$
\sum_{\substack{\mathbf{a} \in \mathcal{P}_{1} \\ L(\mathbf{c}) \geq L_{0}}} e^{S_{p_{1}}(\beta \phi+\psi)(\mathbf{a})} \leq e^{\beta \phi_{g} / 3} \sum_{\substack{\mathbf{a} \in \mathcal{P}_{1} \\\left[\mathbf{a}^{2}-1 \\ p-q^{2}\right] \cap \bar{X}_{J} \neq 0}} e^{S_{p_{1}}(\beta \phi+\psi)(\mathbf{a})},
$$

for all $\beta \geq \max \left(\beta\left(L_{0}\right),\left(6 \# \mathcal{A}\|\psi\|_{\infty}-3 \log (B) /\left(\left|\phi_{g}\right|\left(L_{0}-1\right)-6 \# \mathcal{A}\|\phi\|_{\infty}\right)\right)\right.$, as long as $L_{0}>L_{\phi}:=$ $\left\lceil 6 \# \mathcal{A}\|\phi\|_{\infty} /\left|\phi_{g}\right|\right\rceil+1$.

Let us now consider the set $\mathcal{P}_{2}:=\left\{\mathbf{a} \in \mathcal{P}_{1}: L(\mathbf{a}) \leq L_{\phi}:=\left\lceil 6 \# \mathcal{A}\|\phi\|_{\infty} /\left|\phi_{g}\right|\right\rceil+1\right\}$. For $b_{i}, b_{i}^{\prime} \in \mathcal{A}$ and $m, L \in \mathbb{N}$ fixed, consider the set of all periodic points in $\mathcal{P}_{2}$ whose associated circuit contains a segment $b_{i-1} \rightsquigarrow b_{i-1}^{\prime} \cdot a_{i} \rightsquigarrow a_{i}^{\prime} \cdot b_{i} \rightsquigarrow b_{i}^{\prime}$ of length $L$, with a incursion $a_{i} \rightsquigarrow a_{i}^{\prime}$ of length $m$ into a non-heavy component. If we replace one such segment $b_{i-1} \rightsquigarrow b_{i-1}^{\prime} \cdot a_{i} \rightsquigarrow a_{i}^{\prime} \cdot b_{i} \rightsquigarrow b_{i}^{\prime}$ by a segment $b_{i-1} \rightsquigarrow d_{i-1} \cdot c_{i} \rightsquigarrow c_{i}^{\prime} \cdot d_{i} \rightsquigarrow b_{i}^{\prime}$ of length $L$, such that $c_{i} \rightsquigarrow c_{i}^{\prime}$ lies in $\mathcal{G}_{J}$ and such that $\left|c_{i} \rightsquigarrow c_{i}^{\prime}\right| \geq m-2 \# \mathcal{A}$, then we obtain

$$
\begin{aligned}
\phi\left(b_{i-1} \rightsquigarrow b_{i-1}^{\prime} \cdot a_{i} \rightsquigarrow a_{i}^{\prime} \cdot b_{i} \rightsquigarrow b_{i}^{\prime}\right) \leq & \phi\left(b_{i-1} \rightsquigarrow d_{i-1} \cdot c_{i} \rightsquigarrow c_{i}^{\prime} \cdot d_{i} \rightsquigarrow b_{i}^{\prime}\right)+2 \#\left(L_{\phi}+\mathcal{A}\right)\|\phi\|_{\infty}, \\
\psi\left(b_{i-1} \rightsquigarrow b_{i-1}^{\prime} \cdot a_{i} \rightsquigarrow a_{i}^{\prime} \cdot b_{i} \rightsquigarrow b_{i}^{\prime}\right) \leq & \psi\left(b_{i-1} \rightsquigarrow d_{i-1} \cdot c_{i} \rightsquigarrow c_{i}^{\prime} \cdot d_{i} \rightsquigarrow b_{i}^{\prime}\right) \\
& +2\left(L_{\phi}+3 \# \mathcal{A}\right)\|\psi\|_{\infty}+\psi\left(a_{i} \rightsquigarrow a_{i}^{\prime}\right)-\psi\left(c_{i} \rightsquigarrow c_{i}^{\prime}\right) .
\end{aligned}
$$

Following this prescription we replace all the occurrences of incursions in non-heavy components of length $m \geq \epsilon p_{1}$ by incursions into $\bar{X}_{J}$ of about the same length, and taking into account that 
$P\left(\psi \mid \bar{X}_{J}\right)=P(\psi \mid \bar{X})=0$, we obtain

$$
\begin{aligned}
\sum_{\mathbf{a} \in \mathcal{P}_{2}} e^{S_{p_{1}}(\beta \phi+\psi)(\mathbf{a})} & \leq \sum_{\substack{\mathbf{a} \in \mathcal{P}_{2} \\
\left|a_{i} \rightsquigarrow a_{i}^{\prime}\right|>\epsilon p_{1} \Rightarrow a_{i} \rightsquigarrow a_{i}^{\prime} \text { in } \mathcal{G}_{J}}} e^{S_{p_{1}}(\beta \phi+\psi)(\mathbf{a})} \sum_{k=0}^{L_{\phi}}\left(\begin{array}{c}
L_{\phi} \\
k
\end{array}\right)\left(e^{\left(\epsilon p_{1}-2 \# \mathcal{A}\right)\left(P\left(\psi \mid \bar{X}^{\prime}\right)-P(\psi \mid \bar{X})\right)+r_{\psi}+\beta r_{\phi}}\right)^{k} \\
& \leq\left(1+e^{\left(\epsilon p_{1}-2 \# \mathcal{A}\right) P\left(\psi \mid \bar{X}^{\prime}\right)+r_{\psi}+\beta r_{\phi}}\right)^{L_{\phi}} \sum_{\substack{\mathbf{a} \in \mathcal{P}_{2} \\
\left|a_{i} \rightsquigarrow a_{i}^{\prime}\right|>\epsilon p_{1} \Rightarrow a_{i} \rightsquigarrow a_{i}^{\prime} \text { in } \mathcal{G}_{J}}} e^{S_{p_{1}}(\beta \phi+\psi)(\mathbf{a})}
\end{aligned}
$$

where $r_{\psi}:=\log (\# \mathcal{A})+\log \left(D_{\psi}\right)+2\left(L_{\phi}+3 \# \mathcal{A}\right)\|\psi\|_{\infty}, r_{\phi}:=2\left(L_{\phi}+\# \mathcal{A}\right)\|\phi\|_{\infty}$, and $\bar{X}^{\prime}=\bar{X} \backslash$ $\cup_{K=1}^{N} \bar{X}_{K}$. Since $P\left(\psi \mid \bar{X}^{\prime}\right)<0$, then we have follows that

$$
\begin{aligned}
\sum_{\mathbf{a} \in \mathcal{P}_{2}} e^{S_{p_{1}}(\beta \phi+\psi)(\mathbf{c})} & \leq\left(1+2 L_{\phi} e^{\left(\epsilon p_{1}-2 \# \mathcal{A}\right) P\left(\psi \mid \bar{X}^{\prime}\right)+r_{\psi}+\beta r_{\phi}}\right) \sum_{\substack{\left|a_{i} \rightsquigarrow a_{i}^{\prime}\right|>\epsilon p_{1} \Rightarrow a_{i} \rightsquigarrow a_{i}^{\prime} \text { in } \mathcal{G}_{J}\\
}} e^{S_{p_{1}}(\beta \phi+\psi)(\mathbf{a})} \\
& \leq\left(1+e^{\beta \frac{\phi g}{3}}\right) \sum_{\substack{\mathbf{a} \in \mathcal{P}_{2} \\
\left|a_{i} \rightsquigarrow a_{i}^{\prime}\right|>\epsilon p_{1} \Rightarrow a_{i} \rightsquigarrow a_{i}^{\prime} \text { in } \mathcal{G}_{J}}} e^{S_{p_{1}}(\beta \phi+\psi)(\mathbf{c})}
\end{aligned}
$$

as long as $\epsilon p_{1}>\left(\beta\left(\left|\phi_{g}\right| / 3+r_{\phi}\right)+\log \left(2 L_{\phi}\right)+2 \# \mathcal{A}\left|P\left(\psi \mid \bar{X}^{\prime}\right)\right|+r_{\phi}\right) /\left|P\left(\psi \mid \bar{X}^{\prime}\right)\right|$. We ensure this by taking $\epsilon=q^{-1} \geq e^{-\beta \eta}$ and $\beta$ larger than a convenient $\beta(\eta)$.

Let us group the periodic points in

$$
\mathcal{P}_{3}:=\left\{\mathbf{a} \in \mathcal{P}_{2}:\left|a_{i} \rightsquigarrow a_{i}^{\prime}\right| \geq \epsilon p_{1}=2 q+\left|a \rightarrow a^{\prime}\right| / q \Rightarrow a_{i} \rightsquigarrow a_{i}^{\prime} \text { in } \cup_{K \leq N_{\phi}} \mathcal{G}_{K}\right\},
$$

by classes $\mathcal{Q}:=\mathcal{Q}\left(b_{i} \rightsquigarrow b_{i}^{\prime}, a_{i} \rightsquigarrow a_{i}^{\prime} \in \bigcup_{K=N_{\phi}+1}^{N_{\phi}} \mathcal{G}_{K}, a_{i}, a_{i}^{\prime} \in \bigcup_{K=N_{\phi}+1}^{N_{\phi}} \overline{\mathcal{A}}_{K}, k_{0}\right)$ defined by the excursion segments $b_{i} \rightsquigarrow b_{i}^{\prime}$, the incursion into non-heavy components $a_{i} \rightsquigarrow a_{i}^{\prime}$, the incursion input-output vertices $a_{i}$ and $a_{i}^{\prime}$ into heavy components, and the location $k_{0}$ of the origin in the first incursion segment. By definition, two points $\mathbf{a}, \mathbf{a}^{\prime}$ in the same class are such that $S_{p_{1}} \phi(\mathbf{a})=S_{p_{1}} \phi\left(\mathbf{a}^{\prime}\right)$. Now, for each $\mathbf{a} \in \mathcal{P}_{3}$ we have

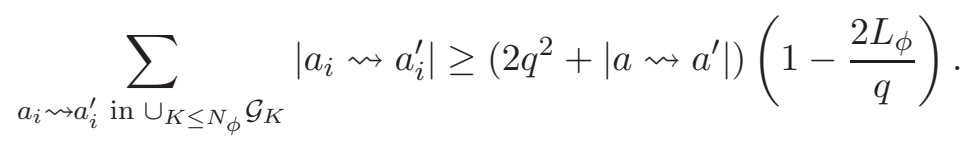

Let $p *=\operatorname{lcm}\left(p_{K}: 1 \leq K \leq N\right)$ and consider a refinement $\mathcal{Q}=\bigsqcup \mathcal{Q}_{\left\{m_{i}: i=1, \ldots, \kappa\right\}}$ of a particular class $\mathcal{Q}:=\mathcal{Q}\left(b_{i} \rightsquigarrow b_{i}^{\prime}, a_{i} \rightsquigarrow a_{i}^{\prime} \in \bigcup_{K=N_{\phi}+1}^{N_{\phi}} \mathcal{G}_{K}, a_{i}, a_{i}^{\prime} \in \bigcup_{K=N_{\phi}+1}^{N_{\phi}} \overline{\mathcal{A}}_{K}, k_{0}\right)$ in subclasses $\mathcal{Q}_{\left\{m_{i}: i=1, \ldots, \kappa\right\}} \subset$ $\mathcal{Q}$ defined by the data of the lengths $m_{i}=\left|a_{i} \rightsquigarrow a_{i}^{\prime}\right|$ of the incursions into heavy components.

A particular subclass $\mathcal{Q}_{\left\{m_{i}: i=1, \ldots, \kappa\right\}} \subset \mathcal{Q}$ contributes with

$$
\begin{aligned}
\sum_{\mathbf{a} \in \mathcal{Q}_{\left\{m_{i}: i=1, \ldots, \kappa\right\}}} e^{S_{p_{1}}(\beta \phi+\psi)(\mathbf{a})} & =e^{\beta \phi\left(C^{\prime}\right)+\psi(\tilde{C})} \prod_{i=1}^{\kappa^{\prime}} \mathcal{M}_{\psi, J_{i}}^{m_{i}}\left(a_{i}, a_{i}^{\prime}\right) \\
& =e^{ \pm \kappa^{\prime} C_{\psi}} e^{\beta \phi\left(C^{\prime}\right)} e^{\psi(\tilde{C})} \prod_{i=1}^{\kappa^{\prime}} \mathbf{v}_{J_{i}}\left(a_{i}\right) \mathbf{w}_{J_{i}}\left(a_{i}^{\prime}\right)
\end{aligned}
$$


to the sum $\sum_{\mathbf{a} \in \mathcal{P}_{2}} e^{S_{p_{1}}(\beta \phi+\psi)(\mathbf{a})}$. Here $C^{\prime}:=C^{\prime}(\mathcal{Q})$ is the circuit defined by the concatenation of the excursions, the incursion into non-heavy components, and path segments $a_{i} \rightarrow a_{i}^{\prime}$ in $\cup_{K \leq N_{\phi}} \mathcal{G}_{K}$ of minimal length. The complement $\tilde{C}:=\tilde{C}(\mathcal{Q})$ is a disjoint union of path segments, formed by the concatenation of all the excursions and the incursion into non-heavy components. The constant $\kappa^{\prime}:=\kappa^{\prime}(\mathcal{Q})<\kappa:=\kappa(\mathcal{Q})$ is the number of times a periodic point in the chosen collection visits a heavy component. Hence, all the subclasses $\mathcal{Q}_{\left\{m_{i}: i=1, \ldots, \kappa\right\}} \subset \mathcal{Q}$ contribute with about the same amount to the $\operatorname{sum} \sum_{\mathbf{a} \in \mathcal{P}_{2}} e^{S_{p_{1}}(\beta \phi+\psi)(\mathbf{a})}$. Their contributions differ at most by a factor in the range $\exp \left( \pm 2 \kappa^{\prime} C_{\psi}\right)=\exp \left( \pm 2 L_{\phi} C_{\psi}\right)$.

Now, to each subclass $\mathcal{Q}_{\left\{m_{i}: i=1, \ldots, \kappa\right\}} \subset \mathcal{Q}$ such that $\min \left(k_{0}, m_{1}-k_{0}\right)<q$ (remember that $k_{0}$ is the location of the origin with respect to the first incursion), there are at least

$$
\frac{1}{2 q p *}\left(\frac{\left(2 q^{2}+\left|a \rightsquigarrow a^{\prime}\right|\right)\left(1-2 L_{\phi} / q\right)}{L_{\phi}}-2 q\right) \geq \frac{q}{2 L_{\phi} p^{*}}
$$

subclasses $\mathcal{Q}_{\left\{m_{i}: i=1, \ldots, \kappa\right\}} \subset \mathcal{Q}$ with $\min \left(k_{0}, m_{1}-k_{0}\right) \geq q$, for all $q$ sufficiently large. Indeed, we can increase the length of the first incursion at both sides of the origin by decreasing the length of another incursions. The size of this length change has to be a common multiple of the periods of the heavy components involved. The decrease of length can be done for length sufficiently large. Each length increase can be associated to at most $2 q$ subclasses with $m_{1} \leq 2 q$. All these lengths changes can be done for all large values of $q$, corresponding to values of $\beta$ greater than a convenient $\beta\left(p^{*}\right)$.

Taking into account that all subclasses $\mathcal{Q}_{\left\{m_{i}: i=1, \ldots, \kappa\right\}} \subset \mathcal{Q}$ contribute with about the same amount to the sum $\sum_{\mathbf{a} \in \mathcal{P}_{2}} e^{S_{p_{1}}(\beta \phi+\psi)(\mathbf{a})}$ (38), and since the majority of those subclasses are such that $\min \left(k_{0}, m_{1}-k_{0}\right) \geq q(39)$, then, since $\eta>-\phi_{g} / 4$ we have

$$
\begin{aligned}
& \sum_{\mathbf{a} \in \mathcal{P}_{3}} e^{S_{p_{1}}(\beta \phi+\psi)(\mathbf{a})}=\sum_{\substack{b_{i} \rightsquigarrow b_{i}^{\prime}, a_{i} \rightsquigarrow a_{i}^{\prime} \in \cup_{K=N_{\phi}+1}^{N_{\phi}} \\
a_{i}, a_{i}^{\prime} \in \cup_{K=N_{\phi}+1}^{N_{\phi}}, \overline{\mathcal{A}}_{K}, k_{0}}} \sum_{m_{i}=\left|a_{i} \rightsquigarrow a_{i}^{\prime}\right| \mathbf{a} \in \mathcal{Q}_{\left\{m_{i}\right\}}} e^{S_{p_{1}}(\beta \phi+\psi)(\mathbf{a})} \\
& \leq\left(1+e^{2 L_{\phi} C_{\psi}} \frac{2 L_{\phi} p^{*}}{q}\right) \times \\
& \sum_{\substack{b_{i} \rightsquigarrow b_{i}^{\prime}, a_{i} \rightsquigarrow a_{i}^{\prime} \in \cup_{K=N_{\phi}+1}^{N_{\phi}} \\
\mathcal{G}_{K},}} \sum_{\substack{m_{i}=\left|a_{i} \rightsquigarrow a_{i}^{\prime}\right| \\
\min \left(k_{0}, m_{1}-k_{0}\right) \geq q}} \sum_{\mathbf{a} \in \mathcal{Q}_{\left\{m_{i}\right\}}} e^{S_{p_{1}}(\beta \phi+\psi)(\mathbf{a})} \\
& a_{i}, a_{i}^{\prime} \in \cup_{K=N_{\phi}+1}^{N_{\phi}} \overline{\mathcal{A}}_{K}, k_{0} \\
& \leq\left(1+e^{\beta \phi_{g} / 4}\right) \sum_{\substack{\mathbf{a} \in \mathcal{P}_{3} \\
\min \left(k_{0}, m_{1}-k_{0}\right) \geq q}} e^{S_{p_{1}}(\beta \phi+\psi)(\mathbf{a})},
\end{aligned}
$$

for $\beta$ larger than $\max \left(\beta(\eta), \beta\left(p^{*}\right)\right)$. 
We can conclude now: (36), (37) and (40) imply that

$$
\sum_{\mathbf{a} \in \mathcal{P}_{1}} e^{S_{p_{1}}(\beta \phi+\psi)(\mathbf{a})} \leq\left(e^{\beta \phi_{g} / 3}+\left(1+e^{\beta \phi_{g} / 3}\right)\left(1+e^{\beta \phi_{g} / 3}\right)\left(1+e^{\beta \phi_{g} / 4}\right)\right) \sum_{\substack{\mathbf{a} \in \mathcal{P}_{1} \\\left[\mathbf{a}_{p-q}^{-1}\right] \cap \bar{X} \neq \emptyset}} e^{S_{p_{1}}(\beta \phi+\psi)(\mathbf{a})},
$$

for all $\beta \geq \max \left(L_{\phi},\left(6 \# \mathcal{A}\|\psi\|_{\infty}-3 \log (B) /\left(\left|\phi_{g}\right|\left(L_{0}-1\right)-6 \# \mathcal{A}\|\phi\|_{\infty}, \beta(\eta), \beta\left(p^{*}\right)\right)\right.\right.$ whence

$$
\sum_{\mathbf{a} \in \mathcal{P}_{1}} e^{S_{p_{1}}(\beta \phi+\psi)(\mathbf{a})} \leq\left(1+2 e^{\beta \phi_{g} / 4}\right) \sum_{\substack{\mathbf{a} \in \mathcal{P}_{1} \\\left[\mathbf{c}_{p-q}^{q-1}\right] \cap \bar{X} \neq \emptyset}} e^{S_{p_{1}}(\beta \phi+\psi)(\mathbf{a})},
$$

which, together with (34) gives the desired result.

\section{B.2. The Spectral Radius.}

Proposition 3 (Convergence of the Spectral Radius). The normalization $\bar{\phi}=P(\psi \mid \bar{X})=0$ implies that $1<\rho_{\beta \phi+\psi} \leq 1+e^{\beta \frac{\phi g}{2}}$ for $\beta$ large enough. (Recall that $\phi_{g}<0$ and it is defined in (12).)

Proof. Since $\mathcal{M}_{\beta \phi+\psi}$ is irreducible, Perron-Frobenius Theorem ensures that

$$
\rho_{\beta \phi+\psi}=\limsup _{p \rightarrow \infty} \sqrt[p]{\operatorname{tr}\left(\mathcal{M}_{\beta \phi+\psi}^{p}\right)}=\limsup _{p \rightarrow \infty} \sqrt[p]{\mathcal{M}_{\beta \phi+\psi}^{p}(a, a)}
$$

for all $a \in \mathcal{A}$. Taking $a \in \overline{\mathcal{A}}_{1}$ and using (23) (which we derived from Corollary 1), we deduce that

$$
\begin{aligned}
\rho_{\beta \phi+\psi}=\limsup _{p \rightarrow \infty} \sqrt[p]{\mathcal{M}_{\beta \phi+\psi}^{p}(a, a)} & >\lim _{k \rightarrow \infty}\left(\mathcal{M}_{\psi, 1}^{k p_{1}}(a, a)\right)^{\frac{1}{k p_{1}}} \\
& \geq \lim _{k \rightarrow \infty}\left(\mathbf{v}_{1}(a) \mathbf{w}_{1}(a)\right)^{\frac{1}{k p_{1}}} e^{-\frac{C_{\psi} \tau_{\psi}^{k}}{k p_{1}}}=1 .
\end{aligned}
$$

For the upper bound we will follow the same technique as in Subsection B.1 above. Let $p=k \bar{p}$ for some $k \in \mathbb{N}$. To each $\mathbf{a} \in \operatorname{Per}_{p}(X)$ we associate a circuit $C(\mathbf{a})$ in $\mathcal{G}_{X}$ which we decompose into its incursion-excursion path segments, $C(\mathbf{a}):=a_{1} \rightsquigarrow a_{1}^{\prime} \cdot b_{1} \rightsquigarrow b_{1}^{\prime} \cdots \cdots a_{\kappa} \rightsquigarrow a_{\kappa}^{\prime} \cdot b_{\kappa} \rightsquigarrow b_{\kappa}^{\prime}$, as previously. We extend $C(\mathbf{c})$ to

$$
\begin{aligned}
& C_{\text {ext }}(\mathbf{a}):=a_{1} \rightsquigarrow a_{1}^{\prime} \cdot b_{1} \rightsquigarrow b_{1}^{\prime} \cdots a_{\kappa} \rightsquigarrow a_{\kappa}^{\prime} \cdot b_{\kappa} \rightsquigarrow b_{\kappa}^{\prime} . \\
& a_{i} \quad a_{\kappa}
\end{aligned}
$$

The complementary circuit $C^{\prime}(\mathbf{a}):=a_{1} \rightarrow a_{1}^{\prime} \cdot b_{1} \rightsquigarrow b_{1}^{\prime} \cdots \cdots a_{i} \rightarrow a_{i}^{\prime} \cdot b_{i} \rightsquigarrow b_{i}^{\prime} \cdots \cdots a_{\kappa} \rightarrow a_{\kappa}^{\prime} \cdot b_{\kappa} \rightsquigarrow b_{\kappa}^{\prime}$, does not include any circuit maximizing $\phi$. As shown in Subsection B.1, we have the upper bounds

$$
\phi(C(\mathbf{a})) \leq\left|C^{\prime}(\mathbf{a})\right| \phi_{g}, \quad \psi(C(\mathbf{a})) \leq \sum_{i=1}^{\kappa} \psi\left(a_{i} \rightsquigarrow a_{i}^{\prime}\right)+(2 \# \mathcal{A}+1)\left|C^{\prime}(\mathbf{a})\right|\|\psi\|_{\infty} .
$$

We first group the periodic points in $\operatorname{Per}_{p}(X)$ according to the number of its incursion-excursion path segments, then we refine the groups so obtained by considering the end sites $n_{1}<n_{1}^{\prime}<$ 
$\cdots<n_{\kappa}<n_{\kappa}^{\prime}$ of the incursion segments. By doing so, and taking into account that $P(\psi \mid \bar{X})=$ $P\left(\psi \mid \bar{X}_{1}\right)=0$, we obtain

$$
\begin{aligned}
\sum_{\mathbf{a} \in \operatorname{Per}_{p}(X)} e^{S_{p}(\beta \phi+\psi)(\mathbf{a})} & \leq \sum_{\kappa=0}^{\lfloor p / 2\rfloor} \sum_{\substack{\mathbf{a} \in \operatorname{Per}(X) \\
\kappa(\mathbf{a})=\kappa}} e^{\left(\beta \phi_{g}+(2 \# \mathcal{A}+1)\|\psi\|_{\infty}\right)\left|C^{\prime}(\mathbf{a})\right|} \prod_{i=1}^{\kappa} e^{\psi\left(a_{i} \approx a_{i}^{\prime}\right)} \\
& \leq \sum_{\kappa=0}^{\lfloor p / 2\rfloor} e^{\left(\beta \phi_{g}+(2 \# \mathcal{A}+1)\|\psi\|_{\infty}+\log (\# \mathcal{A})\right) 2 \kappa} \sum_{n_{1}<n_{1}^{\prime}<\cdots<n_{\kappa}<n_{\kappa}^{\prime}} \prod_{i=2}^{\kappa} K_{\psi} \operatorname{tr}\left(\mathcal{M}_{\psi, 1}^{n_{i}^{\prime}-n_{i}}\right) \\
& \leq \sum_{\kappa=0}^{\lfloor p / 2\rfloor}\left(\begin{array}{c}
p \\
2 \kappa
\end{array}\right) e^{\left(\beta \phi_{g}+(\# \mathcal{A}+1)\|\psi\|_{\infty}+\log (\# \mathcal{A})\right) 2 \kappa} K_{\psi}^{\kappa} \\
& \leq \sum_{\kappa=0}^{p}\left(\begin{array}{l}
p \\
\kappa
\end{array}\right) e^{\left(\beta \phi_{g}+(2 \# \mathcal{A}+1)\|\psi\|_{\infty}+\log (\# \mathcal{A})+\log \left(K_{\psi} / 2\right) \kappa\right.}
\end{aligned}
$$

for all $\beta \geq(2 \# \mathcal{A}+1)\|\psi\|_{\infty}+\log (\# \mathcal{A}) /\left|\phi_{g}\right|$. Here we bound the sums of factors $e^{\psi\left(a_{i} \rightsquigarrow a_{i}^{\prime}\right)}$ by a constant multiple of $\operatorname{tr}\left(\mathcal{M}_{\psi, 1}^{n_{i}^{\prime}-n_{i}}\right)$. As before, the constant $K_{\psi} \geq \# \mathcal{A}$ is taken large enough to include the counting of transitive components in $\bar{X}$, and to compensate the differences in trace among transitive components and the difference between lengths $\left|a_{i} \rightsquigarrow a_{i}^{\prime}\right|$ and $n_{i}^{\prime}-n_{i}$ for each $1 \leq i \leq \kappa$. We are also considering the fact that $2 \kappa(\mathbf{a})<\left|C^{\prime}(\mathbf{a})\right|$. Hence, by taking $\beta$ big enough we obtain

$$
\sum_{\mathbf{a} \in \operatorname{Per}_{p}(X)} e^{S_{p}(\beta \phi+\psi)(\mathbf{a})} \leq\left(1+e^{\beta \phi_{g} / 2}\right)^{p}
$$

therefore

$$
\rho_{\beta \phi+\psi}=\limsup _{p \rightarrow \infty} \sqrt[p]{\operatorname{tr}\left(\mathcal{M}_{\beta \phi+\psi}^{p}\right)}=\limsup _{p \rightarrow \infty} \sqrt[p]{\sum_{\mathbf{a} \in \operatorname{Per}_{p}(X)} e^{S_{p}(\beta \phi+\psi)(\mathbf{a})}} \leq 1+e^{\beta \phi_{g} / 2},
$$

and the result follows.

\section{B.3. Excursion Series.}

Proposition 4 (Convergence of the Excursion Series). There exists a constant $D=D(\psi)>0$ such that for each $a \notin \overline{\mathcal{A}}:=\bigsqcup_{J=1}^{N_{\phi}} \overline{\mathcal{A}}_{J}$ we have

$$
\sum_{j=0}^{\infty} \frac{\tilde{\mathcal{M}}_{\beta \phi+\psi}^{j}(a, a)}{\rho_{\beta \phi+\psi}^{j}}=\sum_{j=0}^{\infty} \overline{\mathcal{M}}_{\psi}^{j}(a, a) \pm D e^{\beta \phi_{g} / 3}
$$

for $\beta$ large enough. 
Proof. For the lower bound notice that

$$
\begin{aligned}
\sum_{j=0}^{\infty} \frac{\tilde{\mathcal{M}}_{\beta \phi+\psi}^{j}(a, a)}{\rho_{\beta \phi+\psi}^{j}} & \geq \sum_{j=0}^{\infty} \frac{\tilde{\mathcal{M}}_{\beta \phi+\psi}^{j}(a, a)}{\left(1+e^{\beta \phi_{g} / 2}\right)^{j}} \geq \sum_{j=0}^{\infty}\left(1-e^{\beta \phi_{g} / 2}\right)^{j} \overline{\mathcal{M}}_{\psi}^{j}(a, a) \geq \sum_{j=0}^{\infty}\left(1-j e^{\beta \phi_{g} / 2}\right) \overline{\mathcal{M}}_{\psi}^{j}(a, a) \\
& \geq \sum_{j=0}^{\infty} \overline{\mathcal{M}}_{\psi}^{j}(a, a)-e^{\beta \phi_{g} / 2} \sum_{j=0}^{\infty} j \overline{\mathcal{M}}_{\psi}^{j}(a, a) \geq \sum_{j=0}^{\infty} \overline{\mathcal{M}}_{\psi}^{j}(a, a)-e^{\beta \phi_{g} / 2} \sum_{j=0}^{\infty} j \operatorname{tr}\left(\overline{\mathcal{M}}_{\psi}^{j}\right) .
\end{aligned}
$$

The convergence of the series $\sum_{j=0}^{\infty} j \overline{\mathcal{M}}_{\psi}^{j}(a, a)$ is ensured by the fact that $a \notin \overline{\mathcal{A}}$. The upper bound $\tilde{\mathcal{M}}_{\beta \phi+\psi}^{j}(a, a) \geq \overline{\mathcal{M}}_{\psi}^{j}(a, a)$ is obtained by restricting the sum

$$
\tilde{\mathcal{M}}_{\beta \phi+\psi}^{j}(a, a):=\sum_{\mathbf{b} \in \operatorname{Per}_{j}(X) \cap \tilde{\mathcal{A}}^{j} \cap[a]} e^{S_{j}(\beta \phi+\psi)(\mathbf{b})}
$$

to periodic points maximizing $S_{j} \phi$.

Now, since $\rho_{\beta \phi+\psi}>1$ (by Proposition 3) we obviously have

$$
\sum_{j=0}^{\infty} \frac{\tilde{\mathcal{M}}_{\beta \phi+\psi}^{j}(a, a)}{\rho_{\beta \phi+\psi}^{j}} \leq \sum_{j=0}^{\infty} \tilde{\mathcal{M}}_{\beta \phi+\psi}^{j}(a, a)=\sum_{j=0}^{\infty} \sum_{\mathbf{b} \in \mathcal{P}_{j}} e^{S_{j}(\beta \phi+\psi)(\mathbf{b})},
$$

where $\mathcal{P}_{j}:=\left\{\mathbf{b} \in \operatorname{Per}_{j}(X):\left(\mathbf{b}_{i}, \mathbf{b}_{i+1}\right) \notin \overline{\mathcal{E}}_{\phi} \forall i \in \mathbb{Z}\right\}$. To upper bound the terms $\sum_{\mathbf{b} \in \mathcal{P}_{j}} e^{S_{j}(\beta \phi+\psi)(\mathbf{b})}$ we use an incursion-excursion decomposition of the circuits associated to periodic points, similar to those employed in the proof of the two previous results. For this, let $\mathcal{G}_{\bar{X}}^{\prime}:=\mathcal{G}_{\bar{X}} \backslash \bigsqcup_{J=1}^{N_{\phi}} \mathcal{G}_{J}$ be the digraph associated to the subshift $\bar{X}^{\prime} \subset \bar{X}$ obtained by excluding all the heavy components, and let $\tilde{\mathcal{G}}_{X}$ be the subgraph spanned by the arrows in the complement of $\mathcal{G}_{\bar{X}}$. Then, each $\mathbf{b} \in \mathcal{P}_{j}$ defines a circuit $C(\mathbf{b})$ in $\mathcal{G}_{\bar{X}}^{\prime}+\tilde{\mathcal{G}}_{X}$ which we decompose into its incursion-excursion path segments,

$$
C(\mathbf{b}):=a_{1} \rightsquigarrow a_{1}^{\prime} \cdot b_{1} \rightsquigarrow b_{1}^{\prime} \cdots a_{\kappa} \rightsquigarrow a_{\kappa}^{\prime} \cdot b_{\kappa} \rightsquigarrow b_{\kappa}^{\prime},
$$

where for each $1 \leq i \leq \kappa, a_{i} \rightsquigarrow a_{i}^{\prime}$ is a path in $\mathcal{G}_{\bar{X}}^{\prime}$ and $b_{i} \rightsquigarrow b_{i}^{\prime}$ is a path in $\tilde{\mathcal{G}}_{X}$. We include the two extreme cases $C(\mathbf{b})$ in $\mathcal{G}_{\bar{X}}^{\prime}$ or in $\tilde{\mathcal{G}}_{X}$, by taking $\kappa=0$ and specifying which of these two possibilities holds. As previously, we extend $C(\mathbf{b})$ to $\left[C_{\text {ext }}(\mathbf{b}):=\sum_{i=1}^{\kappa} a_{i} \rightsquigarrow a_{i}^{\prime}+C^{\prime}(\mathbf{b})\right.$, by adding convenient circuits of minimal length in $\mathcal{G}_{\bar{X}}^{\prime}$. The complementary circuit $C^{\prime}(\mathbf{b}):=a_{1} \rightarrow a_{1}^{\prime} \cdot b_{1} \rightsquigarrow b_{1}^{\prime} \cdots \cdots a_{\kappa} \rightarrow$ $a_{\kappa}^{\prime} \cdot b_{\kappa} \rightsquigarrow b_{\kappa}^{\prime}$, does not include any circuit maximizing $\phi$. As in Subsection B.1, we have the upper bounds:

$$
\phi(C(\mathbf{b})) \leq\left|C^{\prime}(\mathbf{b})\right| \phi_{g}, \quad \psi(C(\mathbf{b})) \leq \sum_{i=1}^{\kappa} \psi\left(a_{i} \rightsquigarrow a_{i}^{\prime}\right)+(2 \# \mathcal{A}+1)\|\psi\|_{\infty}\left|C^{\prime}(\mathbf{b})\right| .
$$

Let $\tilde{P}=P\left(\psi \mid \bar{X}^{\prime}\right)<0$ be the topological pressure of $\psi$ restricted to $\bar{X}^{\prime} \subset \bar{X}$, the collection of all the non-heavy transitive components of $\bar{X}$ (which is of course a union of subshifts of finite type). 
Let us suppose that $[a] \cap \bar{X} \neq \emptyset$, then we have

$$
\begin{aligned}
\sum_{\mathbf{b} \in \mathcal{P}_{j}} e^{S_{j}(\beta \phi+\psi)(\mathbf{b}) \leq} & \sum_{\substack{\mathbf{b} \in \mathcal{P}_{j} \\
\kappa(\mathbf{b})=0}} e^{S_{j}(\beta \phi+\psi)(\mathbf{b})} \\
& +\sum_{\kappa=1}^{\lfloor j / 2\rfloor} \sum_{\substack{\mathbf{b} \in \mathcal{P}_{j} \\
\kappa(\mathbf{b})=\kappa}} e^{\left(\beta \phi_{g}+(2 \# \mathcal{A}+1)\|\psi\|_{\infty}\right)\left|C^{\prime}(\mathbf{b})\right|} \prod_{i=1}^{\kappa} e^{\psi\left(a_{i} \cong a_{i}^{\prime}\right)} \\
\leq & \overline{\mathcal{M}}_{\psi}^{j}(a, a)+\sum_{\kappa=1}^{\lfloor j / 2\rfloor}\left(\begin{array}{c}
j \\
2 \kappa
\end{array}\right) e^{\left(\beta \phi_{g}+(2 \# \mathcal{A}+1)\|\psi\|_{\infty}+\log (\# \mathcal{A})\right)(j-m)} K_{\psi}^{\kappa} e^{\tilde{P} m} \\
\leq & \overline{\mathcal{M}}_{\psi}^{j}(a, a)+\sum_{\kappa=1}^{\lfloor j / 2\rfloor}\left(\begin{array}{c}
j \\
2 \kappa
\end{array}\right) e^{\left(\beta \phi_{g}+(\# \mathcal{A}+1)\|\psi\|_{\infty}+\log (\# A)+\log \left(K_{\psi}\right)\right)(j-m)} e^{\tilde{P} m},
\end{aligned}
$$

for each $\beta \geq(2 \# \mathcal{A}+1)\|\psi\|_{\infty}+\log (\# \mathcal{A}) /\left|\phi_{g}\right|$ and $j \geq 1$. Here $m=\sum_{i=1}^{\kappa}\left|a_{i} \rightsquigarrow a_{i}^{\prime}\right| \leq j-\kappa$ and $K_{\psi}$ is a constant which includes the count and compensates the differences among transitive components of $\bar{X}^{\prime}$. Now, since $j-m \geq \kappa$, we have

$$
\begin{aligned}
\sum_{\mathbf{b} \in \mathcal{P}_{j}} e^{S_{j}(\beta \phi+\psi)(\mathbf{b})} & \leq \overline{\mathcal{M}}_{\psi}^{j}(a, a)+\sum_{\kappa=1}^{\lfloor j / 2\rfloor}\left(\begin{array}{c}
j \\
2 \kappa
\end{array}\right) e^{\left(\beta \phi_{g}+(\# \mathcal{A}+1)\|\psi\|_{\infty}+\log (\# A)+\log \left(K_{\psi}\right)\right) \kappa} e^{\tilde{P}(j-\kappa)} \\
& \leq \overline{\mathcal{M}}_{\psi}^{j}(a, a)+\sum_{\kappa=1}^{j}\left(\begin{array}{c}
j \\
\kappa
\end{array}\right)\left(e^{\beta \phi_{g} / 3}\right)^{(2 \kappa)}\left(e^{\tilde{P} / 2}\right)^{(j-2 \kappa)} \\
& \leq \overline{\mathcal{M}}_{\psi}^{j}(a, a)+\left(e^{\beta \phi_{g} / 3}+e^{\tilde{P} / 2}\right)^{k}-e^{j \tilde{P} / 2}
\end{aligned}
$$

for all $\beta$ greater than $\left(|\tilde{P}|+(2 \# \mathcal{A}+1)\|\psi\|_{\infty}+\log (\# \mathcal{A})+\log \left(D_{\psi}\right)\right) /\left|\phi_{g}\right|$ and all $j \geq 1$. With this we finally obtain

$$
\begin{aligned}
\sum_{j=0}^{\infty} \frac{\tilde{\mathcal{M}}_{\beta \phi+\psi}^{j}(a, a)}{\rho_{\beta \phi+\psi}^{j}} & \leq \sum_{j=0}^{\infty} \overline{\mathcal{M}}_{\psi}^{j}(a, a)+\frac{e^{\beta \phi_{g} / 3}+e^{\tilde{P} / 2}}{1-e^{\beta \phi_{g} / 3}-e^{\tilde{P} / 2}}-\frac{e^{\tilde{P} / 2}}{1-e^{\tilde{P} / 2}} \\
& \leq \sum_{j=0}^{\infty} \overline{\mathcal{M}}_{\psi}^{j}(a, a)+\frac{e^{\beta \phi_{g} / 3}}{\left(1-e^{\beta \phi_{g} / 3}-e^{\tilde{P} / 2}\right)\left(1-e^{\tilde{P} / 2}\right)} \\
& \leq \sum_{j=0}^{\infty} \overline{\mathcal{M}}_{\psi}^{j}(a, a)+\frac{2 e^{\beta \phi_{g} / 3}}{\left(1-e^{\tilde{P} / 2}\right)^{2}},
\end{aligned}
$$

for $\beta$ large enough. A similar computation, for the case $[a] \cap \bar{X}=\emptyset$ leads to

$$
\sum_{j=0}^{\infty} \frac{\tilde{\mathcal{M}}_{\beta \phi+\psi}^{j}(a, a)}{\rho_{\beta \phi+\psi}^{j}} \geq \frac{2 e^{\beta \phi_{g} / 3}}{\left(1-e^{\tilde{P} / 2}\right)^{2}}
$$


for the same values of $\beta$. Since in this last case $\overline{\mathcal{M}}_{\psi}^{j}(a, a)=0$ for all $j \in \mathbb{N}$, the result follows by taking

$$
D=2 \max \left(\frac{1}{\left(1-e^{\tilde{P} / 2}\right)^{2}}, \sum_{j=0}^{\infty} j \operatorname{tr}\left(\overline{\mathcal{M}}_{\psi}^{j}\right)\right)
$$

\section{Appendix C. Projective Stability of the Eigensystems}

Proposition 5 (Projective Stability of the Eigensystem). Let $E$ be a finite set (with at least two elements) and let $M, N: E \times E \rightarrow \mathbb{R}^{+}$be irreducible matrices such that $M=e^{ \pm \eta} N$, for some $\eta>0$. Let $\rho_{M}$ the maximal eigenvalue of $M$, and $\mathbf{w}_{M}, \mathbf{v}_{M}$ the associated left and right positive eigenvectors, normalized such that $\mathbf{w}_{M}^{\dagger} \mathbf{v}_{M}=1$. Let $\rho_{N}, \mathbf{w}_{N}$ and $\mathbf{v}_{N}$ the corresponding quantities for $N$. Then we have

$$
\rho_{M}=e^{ \pm \eta} \rho_{N}, \mathbf{w}_{M}=e^{ \pm 2(\# E-1) \eta} \mathbf{w}_{N} \text { and } \mathbf{v}_{M}=e^{ \pm 2(\# E-1) \eta} \mathbf{v}_{N} .
$$

Proof. First notice that the matrices $N$ and $M$ necessarily have the same period. Using Corollary 1 we readily obtain

$$
\rho_{M}=\limsup _{p \rightarrow \infty} \sqrt[p]{\operatorname{tr}\left(M^{p}\right)}=\limsup _{p \rightarrow \infty} \sqrt[p]{\operatorname{tr}\left(e^{ \pm p \eta} N^{p}\right)}=e^{ \pm \eta} \limsup _{p \rightarrow \infty} \sqrt[p]{\operatorname{tr}\left(N^{p}\right)}=e^{ \pm \eta} \rho_{N} .
$$

For the left and right eigenvectors, let $E=\left\{e_{1}, e_{2}, \ldots, e_{\# E}\right\}$ and consider the reduced matrices $M^{\prime}, N^{\prime}:\left\{e_{2}, \ldots, e_{\# E}\right\} \times\left\{e_{2}, \ldots, e_{\# E}\right\} \rightarrow \mathbb{R}^{+}$such that $M^{\prime}\left(e, e^{\prime}\right)=M\left(e, e^{\prime}\right)$ for all $e, e^{\prime} \in$ $\left\{e_{2}, \ldots, e_{\# E}\right\}$, and similarly for $N^{\prime}$. The right eigenvector $\mathbf{v}_{M}$ associated to $\rho_{M}$, normalized such that $\mathbf{v}_{M}\left(e_{1}\right)=1$, corresponds to the unique solution to the system $\left(M^{\prime}-\rho_{M} \mathrm{Id}\right) \mathbf{x}_{M}=\mathbf{y}_{M}$, where Id is the $(\# E-1)$-dimensional identity matrix and where $\mathbf{y}_{M}:\left\{e_{2}, \ldots, e_{\# E}\right\} \rightarrow \mathbb{R}^{+}$is such that $\mathbf{y}_{M}\left(e_{k}\right)=M\left(e_{k}, e_{1}\right)$. This solution can be obtained by using the Cramer's method, so that

$$
\mathbf{x}_{M}\left(e_{k}\right)=\frac{\operatorname{det}\left(M_{k}\right)}{\operatorname{det}\left(M^{\prime}-\rho_{M} \mathrm{Id}\right)} \text { for each } 2 \leq k \leq \# E .
$$

Here $M_{k}$ is obtained from $M^{\prime}-\rho_{M}$ Id by replacing its $(k-1)$-th column by the vector $\mathbf{y}_{M}$. The same procedure can be employed to obtain the right eigenvector $\mathbf{v}_{N}$ associated $\rho_{N}$, and normalized such that $\mathbf{v}_{N}\left(e_{1}\right)=1$, by solving the equation $\left(N^{\prime}-\rho_{N} \mathrm{Id}\right) \mathbf{x}_{N}=\mathbf{y}_{N}$ by the Cramer's method. Now, since $M^{\prime}-\rho_{M} \mathrm{Id}=e^{ \pm \eta}\left(N^{\prime}-\rho_{N} \mathrm{Id}\right), M_{k}=e^{ \pm \eta} N_{k}$, and the determinant is a $(\# E-1)$-homogeneous function, then

$$
\mathbf{x}_{M}\left(e_{k}\right)=\frac{\operatorname{det}\left(M_{k}\right)}{\operatorname{det}\left(M^{\prime}-\rho_{M} \mathrm{Id}\right)}=e^{ \pm 2(\# E-1) \eta} \frac{\operatorname{det}\left(M_{k}\right)}{\operatorname{det}\left(M^{\prime}-\rho_{M} \mathrm{Id}\right)}=e^{ \pm 2(\# E-1) \eta} \mathbf{x}_{N}\left(e_{k}\right)
$$

for each $2 \leq k \leq \# E$, which implies that $\mathbf{v}_{M}=e^{ \pm 2(\# E-1) \eta} \mathbf{v}_{N}$ as long as they are normalized such that $\mathbf{v}_{M}\left(e_{1}\right)=\mathbf{v}_{N}\left(e_{1}\right)$. The argument goes the same for the left eigenvectors $\mathbf{w}_{M}$ and $\mathbf{w}_{N}$ associated to $\rho_{M}$ and $\rho_{N}$ respectively. The proposition is proved. 


\section{Appendix D. Concentration of the Measure on the Heavy Components}

Lemma 4 (Concentration on the Heavy Components). Let $I_{K}:=\left\{\mathbf{a} \in X:\left[\mathbf{a}_{0}\right] \cap \bar{X}_{K} \neq \emptyset\right\}$. Then for $\beta$ large enough we have $\mu_{\beta \phi+\psi}\left(\cup_{K=1}^{N_{\phi}} I_{K}\right) \geq 1-e^{\phi_{g} / 4}$ for $\beta$ large enough.

Proof. According to Proposition 1, for $\beta$ large enough and for $\gamma=s_{\phi}-\phi_{g} / 4$ one has

$$
\begin{aligned}
\mu_{\beta \phi+\psi}[a] & =\mathcal{P}_{\beta \phi+\psi}^{(k \bar{p})}[a] \exp \left( \pm e^{-\beta\left(\gamma-s_{\phi}\right)}\right) \\
& =\frac{\sum_{\mathbf{a} \in \operatorname{Per}_{k \bar{p}}(X) \cap[a]} e^{S_{k \bar{p}}(\beta \phi+\psi)(\mathbf{a})}}{\sum_{\mathbf{a} \in \operatorname{Per}_{k \bar{p}}(X)} e^{S_{k \bar{p}}(\beta \phi+\psi)(\mathbf{a})}} \exp \left( \pm e^{-\beta\left(\gamma-s_{\phi}\right)}\right)
\end{aligned}
$$

for each $a \in \mathcal{A}$, and every $k \bar{p}>e^{\beta \gamma}+1$. Following the arguments developed in Subsection B.1, we will find bounds for the numerator $\sum_{\mathbf{a} \in \operatorname{Per}_{k \bar{p}}(X) \cap[a]} e^{S_{k \bar{p}}(\beta \phi+\psi)(\mathbf{a})}$ when $a \notin \bigsqcup_{J=1}^{N_{\phi}} \overline{\mathcal{A}}_{J}$.

Let $p=k \bar{p}, q=\left\lfloor e^{\eta \beta}\right\rfloor$, with $-\phi_{g} / 4<\eta<-\phi_{g} / 3$, and for each $b, b^{\prime} \in \mathcal{A}$ let $p_{1}=2 q^{2}+\left|b \rightarrow b^{\prime}\right|$, $p_{2}=p-2 q^{2}+\left|b^{\prime} \rightarrow b\right|$ and $p_{0}=\left|b \circlearrowleft b^{\prime}\right|$, where $b \circlearrowleft b^{\prime}$ is a circuit connecting $b$ and $b^{\prime}$, formed by the concatenation of the minimal length paths $b \rightarrow b^{\prime}$ and $b^{\prime} \rightarrow b$. By the same argument developed in Subsection B.1, it follows that

$$
\begin{aligned}
\sum_{\mathbf{a} \in \operatorname{Per}_{p}(X) \cap[a]} e^{S_{p}(\beta \phi+\psi)(\mathbf{a})}=\sum_{b, b^{\prime} \in \mathcal{A}} e^{-S_{p_{0}}(\beta \phi+\psi)\left(b \circlearrowleft b^{\prime}\right)} & \left(\sum_{\mathbf{a} \in \operatorname{Per}_{p_{2}}(X) \cap\left[b^{\prime} \rightarrow b\right]} e^{S_{p_{2}}(\beta \phi+\psi)(\mathbf{a})}\right) \\
& \times\left(\sum_{\mathbf{a} \in \operatorname{Per}_{p_{1}}(X) \cap[a] \cap \sigma^{-q^{2}\left[b \rightarrow b^{\prime}\right]}} e^{S_{p_{1}}(\beta \phi+\psi)(\mathbf{a})}\right) .
\end{aligned}
$$

We focus on the interior sums $\sum_{\mathbf{a} \in \operatorname{Per}_{p_{1}}(X) \cap[a] \cap \sigma^{-q^{2}\left[a \rightarrow a^{\prime}\right]}} e^{S_{p_{1}}(\beta \phi+\psi)(\mathbf{a})}$.

Each periodic point $\mathbf{a} \in \operatorname{Per}_{p_{1}}(X) \cap \sigma^{-q^{2}}\left[a \rightarrow a^{\prime}\right]$ defines a circuit $C(\mathbf{a})$ in $\mathcal{G}_{X}$. As previously, we decompose this circuit into its incursion-excursion path segments,

$$
C(\mathbf{a}):=a_{1} \rightsquigarrow a_{1} \cdot b_{1} \rightsquigarrow b_{1}^{\prime} \cdot \ldots \cdot a_{\kappa} \rightsquigarrow a_{\kappa}^{\prime} \cdot b_{\kappa} \rightsquigarrow b_{\kappa}^{\prime},
$$

and we extend it by adding, for each $1 \leq i \leq \kappa$, a circuit $a_{i} \circlearrowleft a_{i}^{\prime}$ in $\bar{X}$. The extended circuit

$$
C_{\text {ext }}(\mathbf{a})=\sum_{i=1}^{\kappa} a_{i} \rightsquigarrow a_{i}^{\prime}+a_{1} \rightarrow a_{1} \cdot b_{1} \rightsquigarrow b_{1}^{\prime} \cdot \ldots \cdot a_{\kappa} \rightarrow a_{\kappa}^{\prime} \cdot b_{\kappa} \rightsquigarrow b_{\kappa}^{\prime},
$$

is such that $\phi(C(\mathbf{a}))=\phi\left(C_{\text {ext }}(\mathbf{a})\right)$ and $\psi(C(\mathbf{a})) \leq \psi\left(C_{\text {ext }}(\mathbf{a})\right)+2 \kappa \# \mathcal{A}\|\psi\|_{\infty}$.

The complementary circuit $C^{\prime}(\mathbf{a}):=a_{1} \rightarrow a_{1} \cdot b_{1} \rightsquigarrow b_{1}^{\prime} \cdot \ldots \cdot a_{\kappa} \rightarrow a_{\kappa}^{\prime} \cdot b_{\kappa} \rightsquigarrow b_{\kappa}^{\prime}$, does not include any circuit maximizing $\phi$, therefore $\phi\left(C^{\prime}(\mathbf{a})\right) \leq\left|C^{\prime}(\mathbf{a})\right| \phi_{g}$, and once again, we have the upper bounds

$$
\begin{aligned}
& \phi(C(\mathbf{a})) \leq\left|C^{\prime}(\mathbf{a})\right| \times \phi_{g} \\
& \psi(C(\mathbf{a})) \leq \sum_{i=1}^{\kappa} \psi\left(a_{i} \rightsquigarrow a_{i}^{\prime}\right)+(2 \# \mathcal{A}+1)\|\psi\|_{\infty}\left|C^{\prime}(\mathbf{a})\right| .
\end{aligned}
$$


We group the periodic points in $\mathcal{P}_{1}:=\operatorname{Per}_{p_{1}}(X) \cap \sigma^{-q^{2}}\left[b \rightarrow b^{\prime}\right]$ according to the number of its incursion-excursion path segments. By doing so we have

$$
\begin{aligned}
\sum_{\mathbf{a} \in \mathcal{P}_{1}} e^{S_{p_{1}}(\beta \phi+\psi)(\mathbf{a})} & \leq \sum_{\kappa=0}^{\left\lfloor p_{1} / 2\right\rfloor} \sum_{\substack{\mathbf{a} \in \mathcal{P}_{1} \\
\kappa(\mathbf{a})=\kappa}} e^{S_{p_{1}}(\beta \phi+\psi)(\mathbf{a})} \\
& \leq \sum_{\kappa=0}^{\left\lfloor p_{1} / 2\right\rfloor} \sum_{\substack{\mathbf{a} \in \mathcal{P}_{1} \\
\kappa(\mathbf{c})=\kappa}} e^{\left(\beta \phi_{g}+(2 \# \mathcal{A}+1)\|\psi\|_{\infty}\right)\left|C^{\prime}(\mathbf{a})\right|} \prod_{i=1}^{\kappa} e^{\psi\left(a_{i} \rightsquigarrow a_{i}^{\prime}\right)} .
\end{aligned}
$$

Now we group all the periodic points in $\mathbf{a} \in \mathcal{P}_{1}$ with $\kappa(\mathbf{a})=\kappa$ in classes defined by the total length of the complementary circuit, $L(\mathbf{a}):=\left|C^{\prime}(\mathbf{a})\right|$, the relative position of the incursion and excursion segments, and the location of the origin inside the first incursion $a_{1} \rightsquigarrow a_{1}^{\prime}$. We distinguish periodic orbits for which $L(\mathbf{a}) \leq L_{\phi}:=\left\lceil 6 \# \mathcal{A}\|\phi\|_{\infty} /\left|\phi_{g}\right|\right\rceil+1$. Taking into account Theorem 1 , the fact that $P(\psi \mid \bar{X})=0$, and the fact that $p_{1} \leq 2\left\lfloor e^{2 \beta \eta}\right\rfloor+\# \mathcal{A}$, with $-\phi_{g} / 4<\eta<-\phi_{g} / 3$, we obtain

$$
\begin{aligned}
& \sum_{\mathbf{a} \in \mathcal{P}_{1}} e^{S_{p_{1}}(\beta \phi+\psi)(\mathbf{a})} \leq \sum_{\substack{\mathbf{a} \in \mathcal{P}_{1} \\
L(\mathbf{a})<L_{\phi}}} e^{S_{p_{1}}(\beta \phi+\psi)(\mathbf{a})}+\sum_{\kappa=0}^{\lfloor L / 2\rfloor} \sum_{L=L_{\phi}}^{p_{1}} e^{\left(\beta \phi_{g}+(2 \# \mathcal{A}+1)\|\psi\|_{\infty}+\log (\# \mathcal{A})\right) L} p_{1}\left(\begin{array}{c}
p_{1} \\
2 \kappa-1
\end{array}\right) D_{\psi}^{\kappa} \\
& \leq \sum_{\substack{\mathbf{a} \in \mathcal{P}_{1} \\
L(\mathbf{a})<L_{\phi}}} e^{S_{p_{1}}(\beta \phi+\psi)(\mathbf{a})}+\sum_{L=L_{\phi}}^{p_{1}} e^{\left(\beta \phi_{g}+(2 \# \mathcal{A}+1)\|\psi\|_{\infty}+\log (\# \mathcal{A})+\log \left(p_{1}\right)+\log \left(D_{\psi}\right)+e^{-1}\right) L} \\
& \leq \sum_{\substack{\mathbf{a} \in \mathcal{P}_{1} \\
L(\mathbf{c})<L_{\phi}}} e^{S_{p_{1}}(\beta \phi+\psi)(\mathbf{a})}+2 e^{\beta \frac{\phi g}{3} L_{\phi}}
\end{aligned}
$$

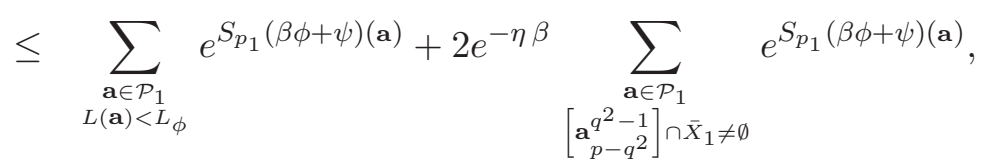

for all $\beta$ larger than a convenient $\beta(L, \eta)$. The constant $D_{\psi}$ is taken large enough to include the counting of transitive components in $\bar{X}$, and to compensate the differences in trace among transitive components, and the difference between lengths $\left|a_{i} \rightsquigarrow a_{i}^{\prime}\right|$ and $m_{i}$ for each $1 \leq i \leq \kappa$. The factor $p_{1}$ takes into account the all the possible locations of the origin with inside the first incursion. We are also considering the fact that $2 \kappa(\mathbf{a}) \leq L(\mathbf{a})$.

Let us now consider the periodic points $\mathcal{P}_{2}:=\left\{\mathbf{a} \in \operatorname{Per}_{p_{1}}(X) \cap \sigma^{-q^{2}}\left[a \rightarrow a^{\prime}\right]: L(\mathbf{a})<L_{\phi}\right\}$. Using exactly the same argument as in Subsection B.1, we deduce that

$$
\sum_{\mathbf{a} \in \mathcal{P}_{2}} e^{S_{p_{1}}(\beta \phi+\psi)(\mathbf{a})} \leq\left(1+e^{\beta \frac{\phi_{g}}{3}}\right) \sum_{\substack{\mathbf{a} \in \mathcal{P}_{2} \\ m_{i}>p_{1} / q \Rightarrow a_{i} \rightsquigarrow a_{i}^{\prime} \text { in }}} e_{K \leq N_{\phi}} \mathcal{G}_{K}{ }^{S_{p_{1}}(\beta \phi+\psi)(\mathbf{a})}
$$

for $\beta$ large enough.

We organize the periodic points in $\mathcal{P}_{3}:=\left\{\mathbf{a} \in \mathcal{P}_{2}:\left|a_{i} \rightsquigarrow a_{i}^{\prime}\right| \geq p_{1} / q \Rightarrow a_{i} \rightsquigarrow a_{i}^{\prime}\right.$ in $\left.\bigsqcup_{K \leq N_{\phi}} \mathcal{G}_{K}\right\}$, by classes defined by fixing the excursion segments $b_{i} \rightsquigarrow b_{i}^{\prime}$, the incursion into non-heavy components 
$a_{i} \rightsquigarrow a_{i}^{\prime}$, and the input-output vertices and lengths of the incursions into heavy components. By definition, two points $\mathbf{a}, \mathbf{a}^{\prime}$ in the same class are such that $S_{p_{1}} \phi(\mathbf{a})=S_{p_{1}} \phi\left(\mathbf{a}^{\prime}\right)$. Now, for each $\mathbf{a} \in \mathcal{P}_{3}$ we have

$$
\sum_{a_{i} \rightsquigarrow a_{i}^{\prime} \text { in } \cup_{K} \mathcal{G}_{K}}\left|a_{i} \rightsquigarrow a_{i}^{\prime}\right| \geq\left(2 q^{2}+\left|b \rightarrow b^{\prime}\right|\right)\left(1-\frac{2 L_{\phi}}{q}\right) .
$$

Let $p *=\operatorname{lcm}\left(p_{K}: 1 \leq K \leq N\right)$ and consider a refinement of a particular class, in subclasses defined by location of the origin. Now, for each subclass such that the segment containing the origin is not included in $\bigsqcup_{K=1}^{N_{\phi}} \mathcal{G}_{K}$, there are at least

$$
\frac{1}{2 q p *}\left(\frac{\left(2 q^{2}+\left|b \rightarrow b^{\prime}\right|\right)\left(1-2 L_{\phi} / q\right)}{L_{\phi}}-2 q\right) \geq \frac{q}{2 p^{*} L_{\phi}}
$$

subclasses such that the segment containing the origin is included in $\cup_{K=1}^{N_{\phi}} \mathcal{G}_{K}$, as long as $q$ is large enough. The argument is exactly the same as the one developed in Subsection B.1 of Appendix B. From (42), (43) and the previous inequality it follows that

$$
\sum_{\substack{\mathbf{a} \in \mathcal{P}_{1} \\ \mathbf{a}_{0} \notin \cup_{K=1}^{N} \overline{\mathcal{A}}_{K}}} e^{S_{p_{1}}(\beta \phi+\psi)(\mathbf{a})} \leq 2 e^{-\beta \eta}\left(3 p^{*} L_{\phi}+1\right) \sum_{\mathbf{c} \in \mathcal{P}_{1}} e^{S_{p_{1}}(\beta \phi+\psi)(\mathbf{a})},
$$

for $\beta$ large enough. Now, using (41) and Proposition 1 we derive the

$$
\sum_{\mathbf{a}_{0} \in \mathcal{A} \backslash \bigsqcup_{K=1}^{N_{\phi}} \overline{\mathcal{A}}_{K}} \mu_{\beta \phi+\psi}\left[\mathbf{a}_{0}\right] \leq 2 e^{-\beta \eta}\left(3 p^{*} L_{\phi}+1\right) \exp \left(e^{-\beta\left(\gamma-s_{\phi}\right)}\right) \leq e^{\beta \phi_{g} / 4}
$$

for $\beta$ large enough, and the result follows.

\section{REFERENCES}

[1] G. Birkhoff, "Projective contraction of a cone equipped with its associated Hilbert metric", Trans. Amer. Math. Soc. 85 (1957), 219-227.

[2] R. Bowen, "Equilibrium States and the Ergodic Theory of Anosov Diffeomorphisms", Lecture Notes in Mathematics 470, 2d Edition, Springer, 2008.

[3] J. Brémont, "Gibbs measures at temperature zero", Nonlinearity 16 (2003) 419-426.

[4] J.-R. Chazottes, L. A. Ramírez and E. Ugalde, "Finite type approximations to Gibbs measures on sofic subshifts", Nonlinearity 18(1) (2005) 445-465.

[5] J.-P. Conze, Y. Guivarc'h, "Croissance des sommes ergodiques et principe variationnel". Unpublished manuscript (1995).

[6] C. Gardner, J. Miekisz, Ch. Radin and A. van Enter, "Fractal Symmetry in an Ising Model", J. Phys. A: Math. Gen. 22(1) (1989) L1019-L1023.

[7] H.-O. Georgii, "Gibbs measures and phase transitions", de Gruyter Studies in Mathematics 9, Walter de Gruyter \& Co., Berlin (1988).

[8] O. Jenkinson, "Geometric barycentres of invariant measures for circle maps", Ergodic Theory 65 Dynamical Systems 21 (2) (2001) 511-532.

[9] R. Leplaideur, "A dynamical proof for the convergence of Gibbs measures at temperature zero", Nonlinearity 18 (2005) 2847-2880.

[10] N. N. Nekhoroshev, "Asymptotics of Gibbs measures in one-dimensional lattice models", Moscow University Mathematical Bulletin 59 (1) (2004) 10-15.

[11] B. Nachtergaele and L. Slegers, "The ground state and its entropy for a class of one-dimensional classical lattice systems", Physica A 149 (1988) 432-446. 
[12] D. Ruelle, "Thermodynamic formalism. The mathematical structures of equilibrium statistical mechanics". Second edition. Cambridge Mathematical Library. Cambridge University Press, Cambridge, 2004.

[13] E. Seneta, "Non-negative Matrices and Markov Chains", Springer Series in Statistics, Springer-Verlag, 1981.

[14] A. C. D. van Enter, R. Fernández, A. Sokal, "Regularity Properties and Pathologies of Position-Space Renormalization-Group Transformations: Scope and Limitations of Gibssian Theory", Journal of Statistical Physics 72 (5/6) (1993), 879-1165. 